\title{
DUAL SZEGÖ PAIRS OF SEQUENCES OF RATIONAL MATRIX-VALUED FUNCTIONS
}

\author{
ANDREAS LASAROW
}

Received 5 January 2005; Revised 9 January 2006; Accepted 12 March 2006

We study certain sequences of rational matrix-valued functions with poles outside the unit circle. These sequences are recursively constructed based on a sequence of complex numbers with norm less than one and a sequence of strictly contractive matrices. We present some basic facts on the rational matrix-valued functions belonging to such kind of sequences and we will see that the validity of some Christoffel-Darboux formulae is an essential property. Furthermore, we point out that the considered dual pairs consist of orthogonal systems. In fact, we get similar results as in the classical theory of Szegö's orthogonal polynomials on the unit circle of the first and second kind.

Copyright (c) 2006 Hindawi Publishing Corporation. All rights reserved.

\section{Introduction}

The theory of orthogonal polynomials is known to have numerous applications in an extensive range of engineering problems. For instance, the important role of Szegö's orthogonal polynomials on the unit circle in circuit and system theory is today well recognized (see, e.g., [1, 29-32, 39] and for discussing the case of matrix polynomials $[9,10,28,38,41],[11$, Section 3.6]).

Starting from different points of view of applications Bultheel, González-Vera, Hendriksen, and Njåstad have formed up a fruitful collaboration and created in the 1990s a comprehensive theory of scalar orthogonal rational functions on the unit circle. In a series of research papers they worked systematically out basic parts of a concept of generalizing essential parts of the classical theory of orthogonal polynomials on the unit circle (see, e.g., [3-7] and probably the first work referring to the rational situation [13] by Džrbašjan).

The present paper is another contribution generalizing this topic to the case of orthogonal rational matrix-valued functions on the unit circle and continues the line of investigations stated in [25-27]. The main objective of this paper is to discuss some dual pairs of sequences of rational matrix-valued functions which are recursively constructed based on a sequence of complex numbers with norm less than one and a sequence of strictly

Hindawi Publishing Corporation

International Journal of Mathematics and Mathematical Sciences

Volume 2006, Article ID 23723, Pages 1-37

DOI 10.1155/IJMMS/2006/23723 
contractive matrices. The recurrence relations defining such pairs are natural generalizations to the situation in question of those fulfilling Szegö's orthogonal polynomials of the first and the second kind. Following the idea of Delsarte et al. [9] with respect to the case of orthogonal matrix polynomials, we only use another normalization for the orthogonal functions in the case under consideration as Szegö in his classical work [39].

Throughout the paper let $n$ be a nonnegative integer, let $q$ be a positive integer, let $\mathbb{C}$ denote the set of all complex numbers, let $\mathbb{D}:=\{w \in \mathbb{C}:|w|<1\}$, let $\mathbb{T}:=\{z \in \mathbb{C}:|z|=$ $1\}$, and let $\left(\alpha_{j}\right)_{j=0}^{\infty}$ be a sequence of complex numbers belonging to the open unit disk $\mathbb{D}$. Furthermore, $\mathbf{I}_{q}$ stands for the identity matrix of size $q \times q$ and the zero matrix of size $q \times q$ is denoted by $\mathbf{0}_{q}$.

Similar as in [25-27], we consider modules $\breve{\mathscr{R}}_{\alpha, n}^{q \times q}$ of rational $q \times q$ matrix-valued functions with prescribed poles (using the convention $1 / \overline{0}:=\infty$ ) at most in the set

$$
\mathbb{P}_{\alpha, n}:=\bigcup_{j=0}^{n}\left\{\frac{1}{\overline{\alpha_{j}}}\right\}
$$

in particular, not located on the unit circle $\mathbb{T}$. We will also use the notation

$$
\mathbb{Z}_{\alpha, n}:=\bigcup_{j=0}^{n}\left\{\alpha_{j}\right\} .
$$

In fact, $\breve{\mathscr{R}_{\alpha, n}} \underset{q \times q}{ }$ denotes the set of all complex $q \times q$ matrix-valued functions $X$ which can be represented via

$$
X=\frac{1}{\pi_{\alpha, n}} P
$$

where $P$ is a complex $q \times q$ matrix polynomial of degree not greater than $n$ and where the polynomial $\pi_{\alpha, n}$ of degree not greater than $n+1$ is given by

$$
\pi_{\alpha, n}(u):=\prod_{j=0}^{n}\left(1-\overline{\alpha_{j}} u\right), \quad u \in \mathbb{C} .
$$

Such kind of rational matrix-valued functions are studied in [25-27] in a way with $\alpha_{0}:=0$ but for a larger set $\left\{\alpha_{1}, \alpha_{2}, \ldots\right\}$ of underlying complex numbers. Since the principal object of this paper is to prepare a particular approach to solve an interpolation problem for matrix-valued Carathéodory functions in $\mathbb{D}$, where $\alpha_{0}, \alpha_{1}, \alpha_{2}, \ldots$ coincide with the treated interpolation points, we make this slight modification.

In the classical case, the connection between orthogonal polynomials on $\mathbb{T}$ and Taylor coefficient problems is particularly given by Schur's algorithm (see $[36,37]$ ). Roughly speaking, Schur's algorithm leads to a sequence of numbers, the so-called Schur parameters, to check if the given data in the problem correspond to a holomorphic function in $\mathbb{D}$ which is bounded by one. As discovered later by Geronimus (see [29]), these Schur parameters are closely connected with the parameters introduced by Szegö (see, e.g., [39]) through recurrence relations for orthogonal polynomials on $\mathbb{T}$. In [33], based on some results contained in [6], an analog interrelation between the parameters which appear in 
an algorithm of Schur-type and the parameters which appear in the recurrence relations for orthogonal rational (complex-valued) functions on T is proved and used to solve an interpolation problem of Nevanlinna-Pick type for complex-valued Carathéodory functions in $\mathbb{D}$.

There is a similar connection between orthogonal rational matrix-valued functions and solving certain interpolation problems of Nevanlinna-Pick type for matrix-valued Carathéodory functions (i.e., matrix interpolation problems which are studied with other methods, e.g., in $[2,8,15])$. But it takes more technical effort to verify such a connection in that case. The main task of this paper is to go some steps towards generalizing the results presented in [33] to the matrix case. In fact, we provide particular formulae starting from the recurrence relations for orthogonal rational matrix-valued functions stated in [26]. In a forthcoming work, these formulae will finally play a key role by solving interpolation problems of Nevanlinna-Pick type for matrix-valued Carathéodory functions in $\mathbb{D}$ via orthogonal rational matrix-valued functions including an interrelation between the parameters which appear in the recurrence relations studied in the present paper and the parameters which appear in the algorithm discussed in [24, Section 5].

Similar as in [25, Definition 3.3], here a sequence $\left(X_{j}\right)_{j=0}^{\tau}$ of matrix-valued functions is called a left (resp., right) orthonormal system corresponding to $\left(\alpha_{j}\right)_{j=0}^{\tau}$ and a nonnegative Hermitian $q \times q$ matrix-valued Borel measure $F$ on $\mathbb{T}$ if the following two conditions are satisfied.

(i) For each integer $j \in\{0,1, \ldots, \tau\}$, the function $X_{j}$ belongs to $\breve{\mathscr{R}}_{\alpha, j}^{q \times q}$.

(ii) For all integers $j, k \in\{0,1, \ldots, \tau\}$,

$$
\int_{\mathbb{T}} X_{j}(z) F(\mathrm{~d} z)\left(X_{k}(z)\right)^{*}=\delta_{j k} \mathbf{I}_{q} \quad\left(\operatorname{resp} ., \int_{\mathbb{T}}\left(X_{j}(z)\right)^{*} F(\mathrm{~d} z) X_{k}(z)=\delta_{j k} \mathbf{I}_{q}\right),
$$

where $\delta_{j k}:=1$ if $j=k$ and $\delta_{j k}:=0$ if $j \neq k$.

Recall that a nonnegative Hermitian $q \times q$ Borel measure on $\mathbb{T}$ is a countably additive mapping from the $\sigma$-algebra $\mathfrak{B}_{\mathbb{T}}$ of all Borel subsets of $\mathbb{T}$ into the set of nonnegative Hermitian $q \times q$ matrices. For basic facts on the integration theory with respect to nonnegative Hermitian Borel measures we refer to [35] (see also [23] concerning the special situation of rational matrix-valued functions). Note that a measure $F$ has to fulfill some additional conditions if orthonormal systems of rational matrix-valued functions as above do exist (see, e.g., [25, Corollary 4.4]).

In [27] it is shown that a pair of orthonormal systems corresponding to $\left(\alpha_{j}\right)_{j=0}^{\tau}$ and $F$, that is, a pair $\left[\left(X_{j}\right)_{j=0}^{\tau},\left(Y_{j}\right)_{j=0}^{\tau}\right]$ consisting of a left (resp., right) orthonormal system $\left(X_{j}\right)_{j=0}^{\tau}$ (resp., $\left.\left(Y_{j}\right)_{j=0}^{\tau}\right)$ corresponding to $\left(\alpha_{j}\right)_{j=0}^{\tau}$ and some nonnegative Hermitian $q \times q$ Borel measure $F$ on $\mathbb{T}$, meets some specific recurrence relations. An essential characteristic of these recurrence relations is marked by an intensive interplay between the elements of the left and the right orthonormal systems although the left and the right versions come in without connection to each other per definition. This phenomenon already occurred in the case of matrix polynomials on $\mathbb{T}$ by finding the analogon of Szegö's recursions for that situation (see [9]).

Using a special normalization for the orthonormal systems of rational matrix-valued functions, the recurrence relations stated in [27] gain a simpler structure (see [26]). In 
fact, [26, Theorems 2.11, 3.5, and 3.7] imply a parametrization of these particular pairs $\left[\left(X_{j}\right)_{j=0}^{\tau},\left(Y_{j}\right)_{j=0}^{\tau}\right]$ of orthogonal rational matrix-valued functions in terms of an initial condition and a sequence $\left(\mathbf{E}_{\ell}\right)_{\ell=1}^{\tau}$ of strictly contractive $q \times q$ matrices. These considerations are the starting point for the present paper. The crucial idea here is that we associate to such a pair $\left[\left(X_{j}\right)_{j=0}^{\tau},\left(Y_{j}\right)_{j=0}^{\tau}\right]$ a dual pair $\left[\left(X_{j}^{\#}\right)_{j=0}^{\tau},\left(Y_{j}^{\#}\right)_{j=0}^{\tau}\right]$ which satisfies analog recurrence relations depending on $\left(-\mathbf{E}_{\ell}\right)_{\ell=1}^{\tau}$ instead of $\left(\mathbf{E}_{\ell}\right)_{\ell=1}^{\tau}$. Since this duality concept given by recurrence relations forms the main part in the proofs of the results below (not directly the orthogonality of the underlying systems), we center such dual pairs of sequences of rational matrix-valued functions and we return to some questions concerning the orthogonality only in the last section of the paper.

A brief synopsis is as follows. In Section 2 we introduce the central notations of this paper and explain basics on the recurrence relations defining these dual pairs of sequences of rational matrix-valued functions. By using certain well-known results on Potapov's Jtheory (see, e.g., $[11,12,14,16,34])$ we get in Section 3 some important properties of the rational matrix-valued functions belonging to such special pairs. In fact, the considerations there are motivated by the studies in [17-19, 21, 22] (see [9] and [11, Section 3.6]) on particular matrix polynomials solving Taylor coefficient problems. In Section 4 we will see that the pairs in question fulfill so-called Christoffel-Darboux formulae. As the treatments in Section 5 imply, the realization of such kind of Christoffel-Darboux formulae is in a way also a sufficient condition for rational matrix-valued functions to be dual Szegö pairs of sequences of rational matrix-valued functions. Finally, we extend in Section 6 the investigations stated in [26, Section 3] on the connection between recurrence relations and orthogonality of rational matrix-valued functions including an alternative proof of [26, Theorem 3.5]. The essential new information in Section 6 is that, based on the duality concept introduced here, one has more insight into the structure of the nonnegative Hermitian $q \times q$ Borel measure occurring already in [26, Theorem 3.5]. Following this train of thoughts, we will obtain two particular choices of measures, where the one corresponds to the pair $\left[\left(X_{j}\right)_{j=0}^{\tau},\left(Y_{j}\right)_{j=0}^{\tau}\right]$, the other corresponds to the dual pair $\left[\left(X_{j}^{\#}\right)_{j=0}^{\tau},\left(Y_{j}^{\#}\right)_{j=0}^{\tau}\right]$, and both can be recovered from each other similar as in the special case of orthogonal matrix polynomials on $\mathbb{T}$ (see, e.g., [11, Definition 3.6.10, Proposition 3.6.9, and Lemma 3.6.28]). In particular, the dual pairs of rational matrix-valued functions are modelled on Szegö's classical orthogonal polynomials of the first and the second kind.

\section{Some basic facts}

As the studies in [25-27] (see also [6]) suggest, the following transform of a rational function into another is an essential tool for the consideration on orthonormal systems of rational matrix-valued functions. If $X \in \breve{\mathscr{R}}_{\alpha, n}^{q \times q}$, then the adjoint rational matrix-valued function $X^{[\alpha, n]}$ of $X$ (with respect to the underlying points $\alpha_{0}, \alpha_{1}, \ldots, \alpha_{n} \in \mathbb{D}$ ) is the rational matrix-valued function (belonging to $\breve{\mathscr{R}}_{\alpha, n}^{q \times q}$ as well) which is uniquely determined by the formula

$$
X^{[\alpha, n]}(u):=\frac{1}{u} B_{n}(u)\left(X\left(\frac{1}{\bar{u}}\right)\right)^{*}, \quad u \in \mathbb{C} \backslash\left(\mathbb{P}_{\alpha, n} \cup \mathbb{Z}_{\alpha, n} \cup\{0\}\right)
$$


(cf. [25, Lemma 2.2 and Remark 2.4]), where

$$
B_{n}(u):=\prod_{j=0}^{n} b_{\alpha_{j}}(u)
$$

and where $b_{\alpha_{j}}$ denotes the elementary Blaschke factor corresponding to $\alpha_{j}$, that is,

$$
b_{\alpha_{j}}(u):= \begin{cases}u & \text { if } \alpha_{j}=0, \\ \frac{\overline{\alpha_{j}}}{\left|\alpha_{j}\right|} \frac{\alpha_{j}-u}{1-\overline{\alpha_{j}} u} & \text { if } \alpha_{j} \neq 0 .\end{cases}
$$

Some information on further interrelations between $X^{[\alpha, n]}$ and the underlying function $X$ can be found in [25, Section 2]. Note that the results on adjoint rational matrix-valued functions in [25] are explained relating to the special case $\alpha_{0}=0$. But it is not hard to restate these with their proofs to the present situation. For instance, if $X, Y \in \breve{\mathscr{R}}_{\alpha, n}^{q \times q}$, then also in that case the following properties are fulfilled.

(I) $X^{[\alpha, n]} \in \breve{\mathscr{R}}_{\alpha, n}^{q \times q},\left(X^{[\alpha, n]}\right)^{[\alpha, n]}=X$.

(II) $X^{[\alpha, n]}\left(\alpha_{n}\right)=\mathbf{0}_{q} \Leftrightarrow X \in \breve{\mathscr{R}}_{\alpha, n-1}^{q \times q}$ for $n \neq 0$.

(III) $(X(z)) * Y(z)=X^{[\alpha, n]}(z)\left(Y^{[\alpha, n]}(z)\right)^{*}$ for $z \in \mathbb{T}$.

We study in the following certain sequences of rational matrix-valued functions formed by given sequences of points belonging to $\mathbb{D}$ and of parameters which are strictly contractive matrices. Recall that a complex $q \times q$ matrix $\mathbf{A}$ is said to be contractive (resp., strictly contractive) if $\mathbf{I}_{q}-\mathbf{A}^{*} \mathbf{A}$ is a nonnegative (resp., positive) Hermitian matrix, where $\mathbf{A}^{*}$ denotes the adjoint matrix of $\mathbf{A}$. For instance, the zero matrix $\mathbf{0}_{q}$ of size $q \times q$ is a strictly contractive matrix.

If $\tau$ is a nonnegative integer or $\infty$, if $\left(\mathbf{E}_{\ell}\right)_{\ell=1}^{\tau}$ is a sequence of strictly contractive $q \times q$ matrices, and if $\mathbf{X}_{0}$ and $\mathbf{Y}_{0}$ are nonsingular complex $q \times q$ matrices fulfilling the condition $\mathbf{X}_{0}^{*} \mathbf{X}_{0}=\mathbf{Y}_{0} \mathbf{Y}_{0}^{*}$, then we define sequences of rational matrix-valued functions $\left(X_{j}\right)_{j=0}^{\tau}$ and $\left(Y_{j}\right)_{j=0}^{\tau}$ by the relations

$$
X_{0}(u):=\frac{\sqrt{1-\left|\alpha_{0}\right|^{2}}}{1-\overline{\alpha_{0}} u} \mathbf{X}_{0}, \quad Y_{0}(u):=\frac{\sqrt{1-\left|\alpha_{0}\right|^{2}}}{1-\overline{\alpha_{0}} u} \mathbf{Y}_{0}, \quad u \in \mathbb{C} \backslash \mathbb{P}_{\alpha, 0},
$$

and, for all integers $\ell \in\{1,2, \ldots, \tau\}$ and points $u \in \mathbb{C} \backslash \mathbb{P}_{\alpha, \ell}$, recursively,

$$
\begin{aligned}
& X_{\ell}(u):=\sqrt{\frac{1-\left|\alpha_{\ell}\right|^{2}}{1-\left|\alpha_{\ell-1}\right|^{2}}} \frac{1-\overline{\alpha_{\ell-1}} u}{1-\overline{\alpha_{\ell}} u}\left(\mathbf{I}_{q}-\mathbf{E}_{\ell} \mathbf{E}_{\ell}^{*}\right)^{-1 / 2}\left(b_{\alpha_{\ell-1}}(u) X_{\ell-1}(u)+\mathbf{E}_{\ell} Y_{\ell-1}^{[\alpha, \ell-1]}(u)\right) \text {, }
\end{aligned}
$$

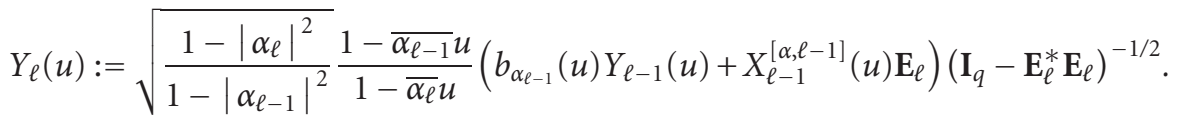

Here and in the sequel $\mathbf{A}^{1 / 2}$ stands for the (unique) nonnegative Hermitian square root of a nonnegative Hermitian $q \times q$ matrix $\mathbf{A}$, the notation $\mathbf{A}^{-1}$ stands for the inverse of a 
nonsingular $q \times q$ matrix $\mathbf{A}$, and hence $\mathbf{A}^{-1 / 2}$ denotes the inverse matrix of the nonnegative Hermitian square root of a positive Hermitian $q \times q$ matrix $\mathbf{A}$ tantamount to the nonnegative Hermitian square root of $\mathbf{A}^{-1}$.

Similar as in [26], we call $\left[\left(X_{j}\right)_{j=0}^{\tau},\left(Y_{j}\right)_{j=0}^{\tau}\right]$ the Szegö pair of rational matrix-valued functions generated by $\left[\left(\alpha_{j}\right)_{j=0}^{\tau} ;\left(\mathbf{E}_{\ell}\right)_{\ell=1}^{\tau} ; \mathbf{X}_{0}, \mathbf{Y}_{0}\right]$. In addition, we consider simultaneously the Szegö pair $\left[\left(X_{j}^{\#}\right)_{j=0}^{\tau},\left(Y_{j}^{\#}\right)_{j=0}^{\tau}\right]$ of rational matrix-valued functions generated by the special choice $\left[\left(\alpha_{j}\right)_{j=0}^{\tau} ;\left(-\mathbf{E}_{\ell}\right)_{\ell=1}^{\tau} ;\left(\mathbf{X}_{0}^{-1}\right)^{*},\left(\mathbf{Y}_{0}^{-1}\right)^{*}\right]$ and call this the dual Szegö pair of $\left[\left(X_{j}\right)_{j=0}^{\tau},\left(Y_{j}\right)_{j=0}^{\tau}\right]$ in the following. In fact, we have

$$
X_{0}^{\#}(u):=\frac{\sqrt{1-\left|\alpha_{0}\right|^{2}}}{1-\overline{\alpha_{0}} u}\left(\mathbf{X}_{0}^{-1}\right)^{*}, \quad Y_{0}^{\#}(u):=\frac{\sqrt{1-\left|\alpha_{0}\right|^{2}}}{1-\overline{\alpha_{0}} u}\left(\mathbf{Y}_{0}^{-1}\right)^{*}, \quad u \in \mathbb{C} \backslash \mathbb{P}_{\alpha, 0},
$$

and, for all integers $\ell \in\{1,2, \ldots, \tau\}$ and points $u \in \mathbb{C} \backslash \mathbb{P}_{\alpha, \ell}$, the recurrence relations

$$
\begin{aligned}
& X_{\ell}^{\#}(u):=\sqrt{\frac{1-\left|\alpha_{\ell}\right|^{2}}{1-\left|\alpha_{\ell-1}\right|^{2}}} \frac{1-\overline{\alpha_{\ell-1}} u}{1-\overline{\alpha_{\ell}} u}\left(\mathbf{I}_{q}-\mathbf{E}_{\ell} \mathbf{E}_{\ell}^{*}\right)^{-1 / 2}\left(b_{\alpha_{\ell-1}}(u) X_{\ell-1}^{\#}(u)-\mathbf{E}_{\ell}\left(Y_{\ell-1}^{\#}\right)^{[\alpha, \ell-1]}(u)\right), \\
& Y_{\ell}^{\#}(u):=\sqrt{\frac{1-\left|\alpha_{\ell}\right|^{2}}{1-\left|\alpha_{\ell-1}\right|^{2}}} \frac{1-\overline{\alpha_{\ell-1}} u}{1-\overline{\alpha_{\ell}} u}\left(b_{\alpha_{\ell-1}}(u) Y_{\ell-1}^{\#}(u)-\left(X_{\ell-1}^{\#}\right)^{[\alpha, \ell-1]}(u) \mathbf{E}_{\ell}\right)\left(\mathbf{I}_{q}-\mathbf{E}_{\ell}^{*} \mathbf{E}_{\ell}\right)^{-1 / 2} \text {. }
\end{aligned}
$$

Remark 2.1. If $\left[\left(X_{j}^{\#}\right)_{j=0}^{\tau},\left(Y_{j}^{\#}\right)_{j=0}^{\tau}\right]$ is the dual Szegö pair of $\left[\left(X_{j}\right)_{j=0}^{\tau},\left(Y_{j}\right)_{j=0}^{\tau}\right]$, then $\left[\left(X_{j}\right)_{j=0}^{\tau},\left(Y_{j}\right)_{j=0}^{\tau}\right]$ is the dual Szegö pair of $\left[\left(X_{j}^{\#}\right)_{j=0}^{\tau},\left(Y_{j}^{\#}\right)_{j=0}^{\tau}\right]$.

The definition of a Szegö pair of rational matrix-valued functions is inspired by the recurrence relations presented in [26, Section 2]. This will be emphasized by the following theorem on particular orthogonal systems of rational matrix-valued functions. A left (resp., right) orthonormal system $\left(X_{j}\right)_{j=0}^{\tau}$ corresponding to $\left(\alpha_{j}\right)_{j=0}^{\tau}$ and some nonnegative Hermitian $q \times q$ matrix-valued Borel measure $F$ on $\mathbb{T}$ is said to be of left (resp., right) Szegö-type if in addition the matrices

$$
\begin{gathered}
\frac{\eta_{\ell} \overline{\eta_{\ell-1}}}{1-\overline{\alpha_{\ell}} \alpha_{\ell-1}}\left(X_{\ell}^{[\alpha, \ell]}\left(\alpha_{\ell-1}\right)\right)^{-1} X_{\ell-1}^{[\alpha, \ell-1]}\left(\alpha_{\ell-1}\right) \\
\left(\text { resp., } \frac{\eta_{\ell} \overline{\eta_{\ell-1}}}{1-\overline{\alpha_{\ell}} \alpha_{\ell-1}} X_{\ell-1}^{[\alpha, \ell-1]}\left(\alpha_{\ell-1}\right)\left(X_{\ell}^{[\alpha, \ell]}\left(\alpha_{\ell-1}\right)\right)^{-1}\right), \quad \ell \in\{1,2, \ldots, \tau\},
\end{gathered}
$$

are positive Hermitian, where the numbers $\eta_{j}, j \in\{0,1, \ldots, \tau\}$, are defined by

$$
\eta_{j}:= \begin{cases}-1 & \text { if } \alpha_{j}=0, \\ \frac{\overline{\alpha_{j}}}{\left|\alpha_{j}\right|} & \text { if } \alpha_{j} \neq 0 .\end{cases}
$$

Note that if there exists a left (resp., right) orthonormal system $\left(Y_{j}\right)_{j=0}^{\tau}$ corresponding to $\left(\alpha_{j}\right)_{j=0}^{\tau}$ and $F$, then one can always choose such a special sequence $\left(X_{j}\right)_{j=0}^{\tau}$ of orthonormal systems (cf. [25, Corollary 4.4] and [26, Remark 2.2]). 
Finally, a pair $\left[\left(X_{j}\right)_{j=0}^{\tau},\left(Y_{j}\right)_{j=0}^{\tau}\right]$ consisting of a left (resp., right) Szegö-type orthonormal system $\left(X_{j}\right)_{j=0}^{\tau}$ (resp., $\left.\left(Y_{j}\right)_{j=0}^{\tau}\right)$ corresponding to $\left(\alpha_{j}\right)_{j=0}^{\tau}$ and $F$ is called a Szegö pair of orthonormal systems corresponding to $\left(\alpha_{j}\right)_{j=0}^{\tau}$ and $F$. Using the same arguments as in [26, Section 2], we get the following statement.

Theorem 2.2. If $\left[\left(X_{j}\right)_{j=0}^{\tau},\left(Y_{j}\right)_{j=0}^{\tau}\right]$ is a Szegö pair of orthonormal systems corresponding to $\left(\alpha_{j}\right)_{j=0}^{\tau}$ and some nonnegative Hermitian $q \times q$ Borel measure $F$ on $\mathbb{T}$, then $\left[\left(X_{j}\right)_{j=0}^{\tau}\right.$, $\left.\left(Y_{j}\right)_{j=0}^{\tau}\right]$ is the Szegö pair of rational matrix-valued functions generated by $\left[\left(\alpha_{j}\right)_{j=0}^{\tau}\right.$; $\left.\left(\mathbf{E}_{\ell}\right)_{\ell=1}^{\tau} ; \mathbf{X}_{0}, \mathbf{Y}_{0}\right]$, where $\mathbf{E}_{\ell}:=\eta_{\ell} \overline{\eta_{\ell-1}}\left(X_{\ell}^{[\alpha, \ell]}\left(\alpha_{\ell-1}\right)\right)^{-1} Y_{\ell}\left(\alpha_{\ell-1}\right)$ for each integer $\ell \in\{1,2, \ldots, \tau\}$, $\mathbf{X}_{0}:=\sqrt{1-\left|\alpha_{0}\right|^{2}} X_{0}\left(\alpha_{0}\right)$, and $\mathbf{Y}_{0}:=\sqrt{1-\left|\alpha_{0}\right|^{2}} Y_{0}\left(\alpha_{0}\right)$.

Example 2.3. If $q=1$ and if $\left[\left(X_{j}\right)_{j=0}^{\tau},\left(Y_{j}\right)_{j=0}^{\tau}\right]$ is the Szegö pair of rational functions formed by an appropriate initial condition and corresponding recurrence relations as above, then there exists $z \in \mathbb{T}$ such that the equality $X_{j}=z Y_{j}$ is fulfilled for each integer $j \in\{0,1, \ldots, \tau\}$. Moreover, if we consider the probably first studied system of orthogonal rational functions (see, e.g., $[13,40]$ ), the so-called Malmquist-Takenaka system $\left(\varphi_{j}\right)_{j=0}^{\tau}$, that is, the rational functions $\varphi_{0}, \varphi_{1}, \ldots, \varphi_{\tau}$ given by

$$
\varphi_{j}(u):= \begin{cases}\frac{\sqrt{1-\left|\alpha_{0}\right|^{2}}}{1-\overline{\alpha_{0}} u} & \text { if } j=0, \\ \frac{\sqrt{1-\left|\alpha_{j}\right|^{2}}}{1-\overline{\alpha_{j}} u} \prod_{k=0}^{j-1} b_{\alpha_{k}}(u) & \text { if } j \neq 0,\end{cases}
$$

for each integer $j \in\{0,1, \ldots, \tau\}$ and point $u \in \mathbb{C} \backslash \mathbb{P}_{\alpha, j}$, then $\left[\left(\varphi_{j}\right)_{j=0}^{\tau},\left(\varphi_{j}\right)_{j=0}^{\tau}\right]$ is the Szegö pair of rational functions generated by $\left[\left(\alpha_{j}\right)_{j=0}^{\tau} ;(0)_{\ell=1}^{\tau} ; 1,1\right]$. Therefore, $\left[\left(\varphi_{j}\right)_{j=0}^{\tau},\left(\varphi_{j}\right)_{j=0}^{\tau}\right]$ is the dual Szegö pair of $\left[\left(\varphi_{j}\right)_{j=0}^{\tau},\left(\varphi_{j}\right)_{j=0}^{\tau}\right]$.

For a strictly contractive $q \times q$ matrix $\mathbf{E}$, we use in the following the notation

$$
\mathbf{H}_{\mathbf{E}}:=\left(\begin{array}{cc}
\left(\mathbf{I}_{q}-\mathbf{E E}^{*}\right)^{-1 / 2} & \mathbf{E}\left(\mathbf{I}_{q}-\mathbf{E}^{*} \mathbf{E}\right)^{-1 / 2} \\
\mathbf{E}^{*}\left(\mathbf{I}_{q}-\mathbf{E E}^{*}\right)^{-1 / 2} & \left(\mathbf{I}_{q}-\mathbf{E}^{*} \mathbf{E}\right)^{-1 / 2}
\end{array}\right)
$$

With a view to $(2.1)$ and the complex $2 q \times 2 q$ matrix-valued functions,

$$
\begin{gathered}
\boldsymbol{\Theta}_{j}:=\left(\begin{array}{cc}
-b_{\alpha_{j}} Y_{j}^{\#} & \left(X_{j}^{\#}\right)^{[\alpha, j]} \\
b_{\alpha_{j}} Y_{j} & X_{j}^{[\alpha, j]}
\end{array}\right), \quad j \in\{0,1, \ldots, \tau\}, \\
\left(\text { resp., } \boldsymbol{\Xi}_{j}:=\left(\begin{array}{cc}
-b_{\alpha_{j}} X_{j}^{\#} & b_{\alpha_{j}} X_{j} \\
\left(Y_{j}^{\#}\right)^{[\alpha, j]} & Y_{j}^{[\alpha, j]}
\end{array}\right)\right), \quad j \in\{0,1, \ldots, \tau\},
\end{gathered}
$$

the recurrence formulae above can be written for all integers $\ell \in\{1,2, \ldots, \tau\}$ and points 
8 Dual Szegö pairs of rational matrix-valued functions

$u \in \mathbb{C} \backslash \mathbb{P}_{\alpha, \ell}$ in matricial form as

$$
\begin{gathered}
\boldsymbol{\Theta}_{\ell}(u)=\sqrt{\frac{1-\left|\alpha_{\ell}\right|^{2}}{1-\left|\alpha_{\ell-1}\right|^{2}} \frac{1-\overline{\alpha_{\ell-1}} u}{1-\overline{\alpha_{\ell}} u} \boldsymbol{\Theta}_{\ell-1}(u) \widehat{\Theta}_{\ell}(u)} \\
\left(\text { resp., } \boldsymbol{\Xi}_{\ell}(u)=\sqrt{\frac{1-\left|\alpha_{\ell}\right|^{2}}{1-\left|\alpha_{\ell-1}\right|^{2}}} \frac{1-\overline{\alpha_{\ell-1}} u}{1-\overline{\alpha_{\ell}} u} \widehat{\Xi}_{\ell}(u) \Xi_{\ell-1}(u)\right),
\end{gathered}
$$

where

$$
\begin{gathered}
\hat{\boldsymbol{\Theta}}_{\ell}(u):=\mathbf{H}_{\mathbf{E}_{\ell}^{*}}\left(\begin{array}{cc}
b_{\alpha_{\ell}}(u) \mathbf{I}_{q} & \mathbf{0}_{q} \\
\mathbf{0}_{q} & \eta_{\ell} \overline{\eta_{\ell-1}} \mathbf{I}_{q}
\end{array}\right), \quad \ell \in\{1,2, \ldots, \tau\}, \\
\left(\text { resp., } \hat{\Xi}_{\ell}(u):=\left(\begin{array}{cc}
b_{\alpha_{\ell}}(u) \mathbf{I}_{q} & \mathbf{0}_{q} \\
\mathbf{0}_{q} & \eta_{\ell} \overline{\eta_{\ell-1}} \mathbf{I}_{q}
\end{array}\right) \mathbf{H}_{\mathbf{E}_{\ell}}\right), \quad \ell \in\{1,2, \ldots, \tau\},
\end{gathered}
$$

and where the numbers $\eta_{j}, j \in\{0,1, \ldots, \tau\}$, are defined by (2.9).

Proposition 2.4. Let $X_{0}, Y_{0}$ be given as in (2.4) with some nonsingular $q \times q$ matrices $\mathbf{X}_{0}, \mathbf{Y}_{0}$ fulfilling $\mathbf{X}_{0}^{*} \mathbf{X}_{0}=\mathbf{Y}_{0} \mathbf{Y}_{0}^{*}$ and let $X_{\ell}, Y_{\ell} \in \breve{\mathscr{R}}_{\alpha, \ell}^{q \times q}$ for each integer $\ell \in\{1,2, \ldots, \tau\}$. Then $\left[\left(X_{j}\right)_{j=0}^{\tau},\left(Y_{j}\right)_{j=0}^{\tau}\right]$ is the Szegö pair of rational matrix-valued functions generated by $\left[\left(\alpha_{j}\right)_{j=0}^{\tau} ;\left(\mathbf{E}_{\ell}\right)_{\ell=1}^{\tau} ; \mathbf{X}_{0}, \mathbf{Y}_{0}\right]$ if and only if for each integer $\ell \in\{1,2, \ldots, \tau\}$ and point $u \in \mathbb{C} \backslash \mathbb{P}_{\alpha, \ell}$ the following backward recurrence relations are satisfied:

$$
\begin{aligned}
& \eta_{\ell} \overline{\eta_{\ell-1}} Y_{\ell}(u)-X_{\ell}^{[\alpha, \ell]}(u) \mathbf{E}_{\ell}=\frac{\left(1-\overline{\alpha_{\ell}} \alpha_{\ell-1}\right)\left(b_{\alpha_{\ell}}(u)-b_{\alpha_{\ell}}\left(\alpha_{\ell-1}\right)\right)}{\sqrt{\left(1-\left|\alpha_{\ell}\right|^{2}\right)\left(1-\left|\alpha_{\ell-1}\right|^{2}\right)}} Y_{\ell-1}(u)\left(\mathbf{I}_{q}-\mathbf{E}_{\ell}^{*} \mathbf{E}_{\ell}\right)^{1 / 2}, \\
& \eta_{\ell} \overline{\eta_{\ell-1}} X_{\ell}(u)-\mathbf{E}_{\ell} Y_{\ell}^{[\alpha, \ell]}(u)=\frac{\left(1-\overline{\alpha_{\ell}} \alpha_{\ell-1}\right)\left(b_{\alpha_{\ell}}(u)-b_{\alpha_{\ell}}\left(\alpha_{\ell-1}\right)\right)}{\sqrt{\left(1-\left|\alpha_{\ell}\right|^{2}\right)\left(1-\left|\alpha_{\ell-1}\right|^{2}\right)}}\left(\mathbf{I}_{q}-\mathbf{E}_{\ell} \mathbf{E}_{\ell}^{*}\right)^{1 / 2} X_{\ell-1}(u) .
\end{aligned}
$$

In particular, if $X_{0}^{\#}, Y_{0}^{\#}$ are defined as in (2.6), if $X_{\ell}^{\#}, Y_{\ell}^{\#} \in \breve{\mathscr{R}}_{\alpha, \ell}^{q \times q}$ for each integer $\ell \in$ $\{1,2, \ldots, \tau\}$, and if $\left[\left(X_{j}\right)_{j=0}^{\tau},\left(Y_{j}\right)_{j=0}^{\tau}\right]$ is the Szegö pair of rational matrix-valued functions generated by $\left[\left(\alpha_{j}\right)_{j=0}^{\tau} ;\left(\mathbf{E}_{\ell}\right)_{\ell=1}^{\tau} ; \mathbf{X}_{0}, \mathbf{Y}_{0}\right]$, then $\left[\left(X_{j}^{\#}\right)_{j=0}^{\tau},\left(Y_{j}^{\#}\right)_{j=0}^{\tau}\right]$ is the dual Szegö pair of $\left[\left(X_{j}\right)_{j=0}^{\tau},\left(Y_{j}\right)_{j=0}^{\tau}\right]$ if and only if for each integer $\ell \in\{1,2, \ldots, \tau\}$ and point $u \in \mathbb{C} \backslash \mathbb{P}_{\alpha, \ell}$ the following backward recurrence relations are satisfied:

$$
\begin{aligned}
& \eta_{\ell} \overline{\eta_{\ell-1}} Y_{\ell}^{\#}(u)+\left(X_{\ell}^{\#}\right)^{[\alpha, \ell]}(u) \mathbf{E}_{\ell}=\frac{\left(1-\overline{\alpha_{\ell}} \alpha_{\ell-1}\right)\left(b_{\alpha_{\ell}}(u)-b_{\alpha_{\ell}}\left(\alpha_{\ell-1}\right)\right)}{\sqrt{\left(1-\left|\alpha_{\ell}\right|^{2}\right)\left(1-\left|\alpha_{\ell-1}\right|^{2}\right)}} Y_{\ell-1}^{\#}(u)\left(\mathbf{I}_{q}-\mathbf{E}_{\ell}^{*} \mathbf{E}_{\ell}\right)^{1 / 2}, \\
& \eta_{\ell} \overline{\eta_{\ell-1}} X_{\ell}^{\#}(u)+\mathbf{E}_{\ell}\left(Y_{\ell}^{\#}\right)^{[\alpha, \ell]}(u)=\frac{\left(1-\overline{\alpha_{\ell}} \alpha_{\ell-1}\right)\left(b_{\alpha_{\ell}}(u)-b_{\alpha_{\ell}}\left(\alpha_{\ell-1}\right)\right)}{\sqrt{\left(1-\left|\alpha_{\ell}\right|^{2}\right)\left(1-\left|\alpha_{\ell-1}\right|^{2}\right)}}\left(\mathbf{I}_{q}-\mathbf{E}_{\ell} \mathbf{E}_{\ell}^{*}\right)^{1 / 2} X_{\ell-1}^{\#}(u) .
\end{aligned}
$$


Proof. Let $\ell \in\{1,2, \ldots, \tau\}$ and $u \in \mathbb{C} \backslash \mathbb{P}_{\alpha, \ell}$. Evidently (cf. [11, Lemma 3.6.32]),

$$
\mathbf{H}_{\mathrm{E}_{\ell}^{*}} \mathbf{H}_{-\mathrm{E}_{\ell}^{*}}=\mathbf{I}_{2 q} \quad\left(\text { resp., } \mathbf{H}_{\mathrm{E}_{\ell}} \mathbf{H}_{-\mathbf{E}_{\ell}}=\mathbf{I}_{2 q}\right)
$$

is satisfied. Therefore, (2.13) is equivalent to the relation

$$
\begin{gathered}
\left(\begin{array}{cc}
-Y_{\ell}^{\#}(u) & \overline{\eta_{\ell}} \eta_{\ell-1}\left(X_{\ell}^{\#}\right)^{[\alpha, \ell]}(u) \\
Y_{\ell}(u) & \overline{\eta_{\ell}} \eta_{\ell-1} X_{\ell}^{[\alpha, \ell]}(u)
\end{array}\right) \mathbf{H}_{-\mathbf{E}_{\ell}^{*}}=\sqrt{\frac{1-\left|\alpha_{\ell}\right|^{2}}{1-\left|\alpha_{\ell-1}\right|^{2}}} \frac{1-\overline{\alpha_{\ell-1}}}{1-\overline{\alpha_{\ell}} u} \Theta_{\ell-1}(u) \\
\left(\text { resp., } \mathbf{H}_{-\mathbf{E}_{\ell}}\left(\begin{array}{cc}
-X_{\ell}^{\#}(u) & X_{\ell}(u) \\
\overline{\eta_{\ell}} \eta_{\ell-1}\left(Y_{\ell}^{\#}\right)^{[\alpha, \ell]}(u) & \overline{\eta_{\ell}} \eta_{\ell-1} Y_{\ell}^{[\alpha, \ell]}(u)
\end{array}\right)=\sqrt{\frac{1-\left|\alpha_{\ell}\right|^{2}}{1-\left|\alpha_{\ell-1}\right|^{2}}} \frac{1-\overline{\alpha_{\ell-1}} u}{1-\overline{\alpha_{\ell}} u} \Xi_{\ell-1}(u)\right) .
\end{gathered}
$$

Hence, by considering the first column of $\boldsymbol{\Theta}_{\ell-1}(u)$ and the first row of $\boldsymbol{\Xi}_{\ell-1}(u)$, using the identity

$$
\overline{\eta_{\ell}} \eta_{\ell-1} \frac{1-\overline{\alpha_{\ell}} \alpha_{\ell-1}}{1-\left|\alpha_{\ell}\right|^{2}}\left(b_{\alpha_{\ell}}(u)-b_{\alpha_{\ell}}\left(\alpha_{\ell-1}\right)\right)=\frac{1-\overline{\alpha_{\ell-1}} u}{1-\overline{\alpha_{\ell}} u} b_{\alpha_{\ell-1}}(u)
$$

and taking into account property (I) of adjoint rational matrix-valued functions, one can finally conclude the assertion.

Observe that the difference between the backward recurrence relations stated in Proposition 2.4 for a Szegö pair of rational matrix-valued functions and for its dual Szegö pair consists in the different signs in front of the parameters $\mathbf{E}_{\ell}, \ell \in\{1,2, \ldots, \tau\}$, similar to the case of the forward recursions defining such pairs of rational matrix-valued functions.

\section{Connection to Potapov's J-theory}

We will show in this section that one can use Potapov's J-theory (see, e.g., $[11,12,14$, 34]) to obtain some information on the rational functions belonging to dual Szegö pairs. In fact, we get certain formulae which can be considered as a generalization of results on matrix polynomials in [21] (with respect to an approach solving Taylor coefficient problems for matrix-valued Carathéodory functions via orthogonal matrix polynomials) to the rational case.

Recall that if $p$ is a positive integer and if $\mathbf{J}_{1}$ and $\mathbf{J}_{2}$ are complex $p \times p$ signature matrices (i.e., unitary and Hermitian) respectively, then a complex $p \times p$ matrix $\mathbf{A}$ is called $\mathbf{J}_{2}$ $\mathbf{J}_{1}$-contractive (resp., $\mathbf{J}_{2}-\mathbf{J}_{1}$-unitary) when $\mathbf{J}_{2}-\mathbf{A}^{*} \mathbf{J}_{1} \mathbf{A}$ is a nonnegative Hermitian matrix (resp., the zero matrix $\mathbf{0}_{p}$ ). In the particular case $\mathbf{J}_{1}=\mathbf{J}_{2}$ we write shortly $\mathbf{J}_{1}$-contractive (resp., $\mathbf{J}_{1}$-unitary) instead of $\mathbf{J}_{1}-\mathbf{J}_{1}$-contractive (resp., $\mathbf{J}_{1}-\mathbf{J}_{1}$-unitary). The special choice of the $2 q \times 2 q$ signature matrices

$$
\mathbf{j}_{q q}:=\left(\begin{array}{cc}
\mathbf{I}_{q} & \mathbf{0}_{q} \\
\mathbf{0}_{q} & -\mathbf{I}_{q}
\end{array}\right), \quad \mathbf{J}_{q}:=\left(\begin{array}{cc}
\mathbf{0}_{q} & -\mathbf{I}_{q} \\
-\mathbf{I}_{q} & \mathbf{0}_{q}
\end{array}\right)
$$

will be essential in the considerations below. 
In the following $\left[\left(X_{j}\right)_{j=0}^{\tau},\left(Y_{j}\right)_{j=0}^{\tau}\right]$ denotes always the Szegö pair of rational matrixvalued functions generated by some $\left[\left(\alpha_{j}\right)_{j=0}^{\tau} ;\left(\mathbf{E}_{\ell}\right)_{\ell=1}^{\tau} ; \mathbf{X}_{0}, \mathbf{Y}_{0}\right]$, where $\left(\alpha_{j}\right)_{j=0}^{\tau}$ is a sequence of points belonging to $\mathbb{D}$, where $\left(\mathbf{E}_{\ell}\right)_{\ell=1}^{\tau}$ is a sequence of strictly contractive $q \times q$ matrices, and where $\mathbf{X}_{0}, \mathbf{Y}_{0}$ are nonsingular complex $q \times q$ matrices fulfilling $\mathbf{X}_{0}^{*} \mathbf{X}_{0}=\mathbf{Y}_{0} \mathbf{Y}_{0}^{*}$, as well as $\left[\left(X_{j}^{\#}\right)_{j=0}^{\tau},\left(Y_{j}^{\#}\right)_{j=0}^{\tau}\right]$ stands for its dual Szegö pair.

Theorem 3.1. For each integer $j \in\{0,1, \ldots, \tau\}$ and point $u \in \mathbb{C} \backslash \mathbb{P}_{\alpha, j}$,

$$
\begin{aligned}
& \boldsymbol{\Theta}_{j}(u)=\frac{\sqrt{2\left(1-\left|\alpha_{j}\right|^{2}\right)}}{1-\overline{\alpha_{j}} u} \hat{\boldsymbol{\Theta}}_{0}(u) \hat{\boldsymbol{\Theta}}_{1}(u) \cdots \hat{\boldsymbol{\Theta}}_{j}(u), \\
& \boldsymbol{\Xi}_{j}(u)=\frac{\sqrt{2\left(1-\left|\alpha_{j}\right|^{2}\right)}}{1-\overline{\alpha_{j}} u} \hat{\boldsymbol{\Xi}}_{j}(u) \hat{\boldsymbol{\Xi}}_{j-1}(u) \cdots \hat{\boldsymbol{\Xi}}_{0}(u),
\end{aligned}
$$

where $\boldsymbol{\Theta}_{j}(u), \boldsymbol{\Xi}_{j}(u)$ and $\hat{\boldsymbol{\Theta}}_{\ell}(u), \hat{\boldsymbol{\Xi}}_{\ell}(u)$ for $\ell \in\{1,2, \ldots, j\}$ are given by (2.12) and (2.14) as well as

$$
\begin{aligned}
& \hat{\boldsymbol{\Theta}}_{0}(u):=\frac{1-\overline{\alpha_{0}} u}{\sqrt{2\left(1-\left|\alpha_{0}\right|^{2}\right)}} \boldsymbol{\Theta}_{0}(u)=\frac{1}{\sqrt{2}}\left(\begin{array}{cc}
-\left(\mathbf{Y}_{0}^{-1}\right)^{*} & \mathbf{X}_{0}^{-1} \\
\mathbf{Y}_{0} & \mathbf{X}_{0}^{*}
\end{array}\right)\left(\begin{array}{cc}
b_{\alpha_{0}}(u) \mathbf{I}_{q} & \mathbf{0}_{q} \\
\mathbf{0}_{q} & -\eta_{0} \mathbf{I}_{q}
\end{array}\right), \\
& \hat{\Xi}_{0}(u):=\frac{1-\overline{\alpha_{0}} u}{\sqrt{2\left(1-\left|\alpha_{0}\right|^{2}\right)}} \mathbf{\Xi}_{0}(u)=\frac{1}{\sqrt{2}}\left(\begin{array}{cc}
b_{\alpha_{0}}(u) \mathbf{I}_{q} & \mathbf{0}_{q} \\
\mathbf{0}_{q} & -\eta_{0} \mathbf{I}_{q}
\end{array}\right)\left(\begin{array}{cc}
-\left(\mathbf{X}_{0}^{-1}\right)^{*} & \mathbf{X}_{0} \\
\mathbf{Y}_{0}^{-1} & \mathbf{Y}_{0}^{*}
\end{array}\right) \text {. }
\end{aligned}
$$

In particular, if $u \in \mathbb{D}$, then the matrices $\left(\left(1-\overline{\alpha_{j}} u\right) / \sqrt{2\left(1-\left|\alpha_{j}\right|^{2}\right)}\right) \Theta_{j}(u)$ and $\left(\left(\left(1-\overline{\alpha_{j}} u\right) /\right.\right.$ $\left.\left.\sqrt{2\left(1-\left|\alpha_{j}\right|^{2}\right)}\right) \boldsymbol{\Xi}_{j}(u)\right)^{*}$ are $\mathbf{j}_{q q} \mathbf{J}_{q}$-contractive and if $u \in \mathbb{T}$, then $\left(\left(1-\overline{\alpha_{j}} u\right) / \sqrt{2\left(1-\left|\alpha_{j}\right|^{2}\right)}\right)$ $\boldsymbol{\Theta}_{j}(u)$ and $\left(\left(\left(1-\overline{\alpha_{j}} u\right) / \sqrt{2\left(1-\left|\alpha_{j}\right|^{2}\right)}\right) \boldsymbol{\Xi}_{j}(u)\right) *$ are even $\mathbf{j}_{q q}-\mathbf{J}_{q}$-unitary.

Proof. Let $j \in\{0,1, \ldots, \tau\}$ and $u \in \mathbb{C} \backslash \mathbb{P}_{\alpha, j}$. We prove only the expressions with respect to $\Theta_{j}(u)$. Similarly, one can verify the others by using the same arguments. The representation (3.2) of $\boldsymbol{\Theta}_{j}(u)$ is an easy consequence of (2.13) and the choice of $X_{0}, Y_{0}, X_{0}^{\#}$, and $Y_{0}^{\#}$ (according to (2.4) and (2.6)). It remains to prove that if $u \in \mathbb{D}$ (resp., $u \in \mathbb{T}$ ), then the matrix $\left(\left(1-\overline{\alpha_{j}} u\right) / \sqrt{2\left(1-\left|\alpha_{j}\right|^{2}\right)}\right) \boldsymbol{\Theta}_{j}(u)$ is $\mathbf{j}_{q q}-\mathbf{J}_{q}$-contractive (resp., $\mathbf{j}_{q q}-\mathbf{J}_{q}$-unitary). Obviously, we have

$$
\left(\begin{array}{cc}
b_{\alpha_{k}}(u) \mathbf{I}_{q} & \mathbf{0}_{q} \\
\mathbf{0}_{q} & z \mathbf{I}_{q}
\end{array}\right)^{*} \mathbf{j}_{q q}\left(\begin{array}{cc}
b_{\alpha_{k}}(u) \mathbf{I}_{q} & \mathbf{0}_{q} \\
\mathbf{0}_{q} & z \mathbf{I}_{q}
\end{array}\right)=\left(\begin{array}{cc}
\left|b_{\alpha_{k}}(u)\right|^{2} \mathbf{I}_{q} & \mathbf{0}_{q} \\
\mathbf{0}_{q} & -\mathbf{I}_{q}
\end{array}\right), \quad k \in\{0,1, \ldots, j\},
$$

if $z \in \mathbb{T}$ and (cf. [11, Lemma 3.6.32])

$$
\left(\mathbf{H}_{\mathrm{E}_{\ell}^{*}}\right) * \mathbf{j}_{q q} \mathbf{H}_{\mathrm{E}_{\ell}^{*}}=\mathbf{j}_{q q}, \quad \ell \in\{1,2, \ldots, j\},
$$


as well as, since $\mathbf{X}_{0}^{*} \mathbf{X}_{0}=\mathbf{Y}_{0} \mathbf{Y}_{0}^{*}$, furthermore

$$
\left(\begin{array}{cc}
-\left(\mathbf{Y}_{0}^{-1}\right)^{*} & \mathbf{X}_{0}^{-1} \\
\mathbf{Y}_{0} & \mathbf{X}_{0}^{*}
\end{array}\right)^{*} \mathbf{J}_{q}\left(\begin{array}{cc}
-\left(\mathbf{Y}_{0}^{-1}\right)^{*} & \mathbf{X}_{0}^{-1} \\
\mathbf{Y}_{0} & \mathbf{X}_{0}^{*}
\end{array}\right)=2 \mathbf{j}_{q q}
$$

Hence (using [16, Lemma 4]), if $u \in \mathbb{D}$ (resp., $u \in \mathbb{T}$ ) then the matrix $\widehat{\boldsymbol{\Theta}}_{\ell}(u)$ is $\mathbf{j}_{q q^{-}}$ contractive (resp., $\mathbf{j}_{q q}$-unitary) for each $\ell \in\{1,2, \ldots, j\}$ as well as the matrix $\widehat{\boldsymbol{\Theta}}_{0}(u)$ is $\mathbf{j}_{q q}-\mathbf{J}_{q}$-contractive (resp., $\mathbf{j}_{q q}-\mathbf{J}_{q}$-unitary) which implies by virtue of (3.2) that the matrix $\left(\left(1-\overline{\alpha_{j}} u\right) / \sqrt{2\left(1-\left|\alpha_{j}\right|^{2}\right)}\right) \boldsymbol{\Theta}_{j}(u)$ is $\mathbf{j}_{q q}-\mathbf{J}_{q}$-contractive (resp., $\mathbf{j}_{q q}-\mathbf{J}_{q}$-unitary).

Theorem 3.1 yields in view of some well-known results on $\mathbf{j}_{q q}-\mathbf{J}_{q}$-contractive matrices (see, e.g., [16, Lemma 8] and use in addition [11, Lemma 2.1.5]) particularly the following result.

Corollary 3.2. For each integer $j \in\{0,1, \ldots, \tau\}$ and point $u \in \mathbb{D} \cup \mathbb{T}$, the relations $\operatorname{det} X_{j}^{[\alpha, \ell]}(u) \neq 0, \operatorname{det}\left(X_{j}^{\#}\right)^{[\alpha, \ell]}(u) \neq 0, \operatorname{det} Y_{j}^{[\alpha, \ell]}(u) \neq 0$, and $\operatorname{det}\left(Y_{j}^{\#}\right)^{[\alpha, \ell]}(u) \neq 0$ are satisfied. Moreover, for each integer $j \in\{0,1, \ldots, \tau\}$, the real part of

$$
\begin{gathered}
\left(X_{j}^{\#}\right)^{[\alpha, j]}(u)\left(X_{j}^{[\alpha, j]}(u)\right)^{-1}, \quad X_{j}^{[\alpha, j]}(u)\left(\left(X_{j}^{\#}\right)^{[\alpha, j]}(u)\right)^{-1} \\
\left(\operatorname{resp.},\left(Y_{j}^{[\alpha, j]}(u)\right)^{-1}\left(Y_{j}^{\#}\right)^{[\alpha, j]}(u),\left(\left(Y_{j}^{\#}\right)^{[\alpha, j]}(u)\right)^{-1} Y_{j}^{[\alpha, j]}(u)\right),
\end{gathered}
$$

respectively, is a positive Hermitian matrix if $u \in \mathbb{D} \cup \mathbb{T}$ and the matrices

$$
\begin{gathered}
b_{\alpha_{j}}(u)\left(X_{j}^{[\alpha, j]}(u)\right)^{-1} Y_{j}(u), \quad b_{\alpha_{j}}(u)\left(\left(X_{j}^{\#}\right)^{[\alpha, j]}(u)\right)^{-1} Y_{j}^{\#}(u) \\
\left(\operatorname{resp} ., b_{\alpha_{j}}(u) X_{j}(u)\left(Y_{j}^{[\alpha, j]}(u)\right)^{-1}, b_{\alpha_{j}}(u) X_{j}^{\#}(u)\left(\left(Y_{j}^{\#}\right)^{[\alpha, j]}(u)\right)^{-1}\right)
\end{gathered}
$$

are strictly contractive if $u \in \mathbb{D}$ and unitary if $u \in \mathbb{T}$.

Since Corollary 3.2 includes a localization of the zeros of $\operatorname{det} X_{\ell}^{[\alpha, \ell]}, \operatorname{det}\left(X_{\ell}^{\#}\right)^{[\alpha, \ell]}$, $\operatorname{det} Y_{\ell}^{[\alpha, \ell]}$, and $\operatorname{det}\left(Y_{\ell}^{\#}\right)^{[\alpha, \ell]}, \ell \in\{1,2, \ldots, \tau\}$, the next Corollary is an easy conclusion of Proposition 2.4 and (2.13) by the special choice $u=\alpha_{\ell-1}$.

Corollary 3.3. For each integer $\ell \in\{1,2, \ldots, \tau\}$, the parameter $\mathbf{E}_{\ell}$ can be attained via

$$
\begin{aligned}
\mathbf{E}_{\ell} & =\eta_{\ell} \overline{\eta_{\ell-1}}\left(X_{\ell}^{[\alpha, \ell]}\left(\alpha_{\ell-1}\right)\right)^{-1} Y_{\ell}\left(\alpha_{\ell-1}\right)=\eta_{\ell} \overline{\eta_{\ell-1}} X_{\ell}\left(\alpha_{\ell-1}\right)\left(Y_{\ell}^{[\alpha, \ell]}\left(\alpha_{\ell-1}\right)\right)^{-1}, \\
-\mathbf{E}_{\ell} & =\eta_{\ell} \overline{\eta_{\ell-1}}\left(\left(X_{\ell}^{\#}\right)^{[\alpha, \ell]}\left(\alpha_{\ell-1}\right)\right)^{-1} Y_{\ell}^{\#}\left(\alpha_{\ell-1}\right)=\eta_{\ell} \overline{\eta_{\ell-1}} X_{\ell}^{\#}\left(\alpha_{\ell-1}\right)\left(\left(Y_{\ell}^{\#}\right)^{[\alpha, \ell]}\left(\alpha_{\ell-1}\right)\right)^{-1}
\end{aligned}
$$


(cf.Theorem 2.2) and in addition

$$
\begin{aligned}
& \left(\mathbf{I}_{q}-\mathbf{E}_{\ell} \mathbf{E}_{\ell}^{*}\right)^{1 / 2} \\
& \quad=\eta_{\ell} \overline{\eta_{\ell-1}} \frac{\sqrt{\left(1-\left|\alpha_{\ell}\right|^{2}\right)\left(1-\left|\alpha_{\ell-1}\right|^{2}\right)}}{1-\overline{\alpha_{\ell}} \alpha_{\ell-1}}\left(X_{\ell}^{[\alpha, \ell]}\left(\alpha_{\ell-1}\right)\right)^{-1} X_{\ell-1}^{[\alpha, \ell-1]}\left(\alpha_{\ell-1}\right) \\
& \quad=\eta_{\ell} \overline{\eta_{\ell-1}} \frac{\sqrt{\left(1-\left|\alpha_{\ell}\right|^{2}\right)\left(1-\left|\alpha_{\ell-1}\right|^{2}\right)}}{1-\overline{\alpha_{\ell}} \alpha_{\ell-1}}\left(\left(X_{\ell}^{\#}\right)^{[\alpha, \ell]}\left(\alpha_{\ell-1}\right)\right)^{-1}\left(X_{\ell-1}^{\#}\right)^{[\alpha, \ell-1]}\left(\alpha_{\ell-1}\right), \\
& \left(\mathbf{I}_{q}-\mathbf{E}_{\ell}^{*} \mathbf{E}_{\ell}\right)^{1 / 2} \\
& \quad=\eta_{\ell} \overline{\eta_{\ell-1}} \frac{\sqrt{\left(1-\left|\alpha_{\ell}\right|^{2}\right)\left(1-\left|\alpha_{\ell-1}\right|^{2}\right)}}{1-\overline{\alpha_{\ell}} \alpha_{\ell-1}} Y_{\ell-1}^{[\alpha, \ell-1]}\left(\alpha_{\ell-1}\right)\left(Y_{\ell}^{[\alpha, \ell]}\left(\alpha_{\ell-1}\right)\right)^{-1} \\
& =
\end{aligned}
$$

Remark 3.4. For each integer $j \in\{0,1, \ldots, \tau\}$, from Corollary 3.2 we know that $X_{j}^{[\alpha, j]}\left(\alpha_{j}\right)$, $\left(X_{j}^{\#}\right)^{[\alpha, j]}\left(\alpha_{j}\right), Y_{j}^{[\alpha, j]}\left(\alpha_{j}\right)$, and $\left(Y_{j}^{\#}\right)^{[\alpha, j]}\left(\alpha_{j}\right)$ are nonsingular matrices. In particular, property (II) of adjoint rational matrix-valued functions implies that for each integer $\ell \in$ $\{1,2, \ldots, \tau\}$ the functions $X_{\ell}, Y_{\ell}, X_{\ell}^{\#}$, and $Y_{\ell}^{\#}$ belong to $\breve{\mathscr{R}}_{\alpha, \ell}^{q \times q} \backslash \breve{\mathscr{R}}_{\alpha, \ell-1}^{q \times q}$ and that if we put $Z_{j} \in\left\{X_{j}, Y_{j}, X_{j}^{\#}, Y_{j}^{\#}\right\}$ for all integers $j$ and $n$ satisfying $0 \leq j \leq n \leq \tau$, then the system $Z_{0}, Z_{1}, \ldots, Z_{n}$ is both a basis of the right and the left $\mathbb{C}^{q \times q}$-module $\breve{\mathscr{R}}_{\alpha, n}^{q \times q}$ (cf. [25, (2.10)] and [23, Proposition 2.7]).

Proposition 3.5. For each integer $j \in\{0,1, \ldots, \tau\}$ and point $u \in \mathbb{C} \backslash \mathbb{P}{ }_{\alpha, j}$,

$$
\boldsymbol{\Xi}_{j}(u)\left(\begin{array}{cc}
\mathbf{0}_{q} & \mathbf{I}_{q} \\
-\mathbf{I}_{q} & \mathbf{0}_{q}
\end{array}\right) \boldsymbol{\Theta}_{j}(u)=2 \eta_{j} \frac{1-\left|\alpha_{j}\right|^{2}}{\left(1-\overline{\alpha_{j}} u\right)^{2}} B_{j}(u)\left(\begin{array}{cc}
\mathbf{0}_{q} & \mathbf{I}_{q} \\
-\mathbf{I}_{q} & \mathbf{0}_{q}
\end{array}\right),
$$

where $\boldsymbol{\Theta}_{j}(u)$ and $\boldsymbol{\Xi}_{j}(u)$ are defined as in (2.12), the number $\eta_{j}$ is given as in (2.9) concerning $\alpha_{j}$, and $B_{j}(u)$ is given as in (2.2) with respect to $\alpha_{0}, \alpha_{1}, \ldots, \alpha_{j}$.

Proof. Let $j \in\{0,1, \ldots, \tau\}$ and $u \in \mathbb{C} \backslash \mathbb{P}_{\alpha, j}$. Further, let $\eta_{-1}:=-1$. A straightforward calculation yields

$$
\left(\begin{array}{cc}
b_{\alpha_{k}}(u) \mathbf{I}_{q} & \mathbf{0}_{q} \\
\mathbf{0}_{q} & \eta_{k} \overline{\eta_{k-1}} \mathbf{I}_{q}
\end{array}\right)\left(\begin{array}{cc}
\mathbf{0}_{q} & \mathbf{I}_{q} \\
-\mathbf{I}_{q} & \mathbf{0}_{q}
\end{array}\right)\left(\begin{array}{cc}
b_{\alpha_{k}}(u) \mathbf{I}_{q} & \mathbf{0}_{q} \\
\mathbf{0}_{q} & \eta_{k} \overline{\eta_{k-1}} \mathbf{I}_{q}
\end{array}\right)=\eta_{k} \overline{\eta_{k-1}} b_{\alpha_{k}}(u)\left(\begin{array}{cc}
\mathbf{0}_{q} & \mathbf{I}_{q} \\
-\mathbf{I}_{q} & \mathbf{0}_{q}
\end{array}\right)
$$

for each $k \in\{0,1, \ldots, j\}$ and (using [11, Lemma 1.1.12])

$$
\mathbf{H}_{\mathrm{E}_{\ell}}\left(\begin{array}{cc}
\mathbf{0}_{q} & \mathbf{I}_{q} \\
-\mathbf{I}_{q} & \mathbf{0}_{q}
\end{array}\right) \mathbf{H}_{\mathrm{E}_{\ell}^{*}}=\left(\begin{array}{cc}
\mathbf{0}_{q} & \mathbf{I}_{q} \\
-\mathbf{I}_{q} & \mathbf{0}_{q}
\end{array}\right), \quad \ell \in\{1,2, \ldots, j\},
$$


as well as, since $\mathbf{X}_{0}^{*} \mathbf{X}_{0}=\mathbf{Y}_{0} \mathbf{Y}_{0}^{*}$,

$$
\left(\begin{array}{cc}
-\left(\mathbf{X}_{0}^{-1}\right)^{*} & \mathbf{X}_{0} \\
\mathbf{Y}_{0}^{-1} & \mathbf{Y}_{0}^{*}
\end{array}\right)\left(\begin{array}{cc}
\mathbf{0}_{q} & \mathbf{I}_{q} \\
-\mathbf{I}_{q} & \mathbf{0}_{q}
\end{array}\right)\left(\begin{array}{cc}
-\left(\mathbf{Y}_{0}^{-1}\right)^{*} & \mathbf{X}_{0}^{-1} \\
\mathbf{Y}_{0} & \mathbf{X}_{0}^{*}
\end{array}\right)=-2\left(\begin{array}{cc}
\mathbf{0}_{q} & \mathbf{I}_{q} \\
-\mathbf{I}_{q} & \mathbf{0}_{q}
\end{array}\right) .
$$

Consequently, from (3.2) one can finally conclude the assertion.

The next result is an easy consequence of Proposition 3.5, (2.12), and

$$
\left(\begin{array}{cc}
\mathbf{0}_{q} & \mathbf{I}_{q} \\
-\mathbf{I}_{q} & \mathbf{0}_{q}
\end{array}\right)\left(\begin{array}{cc}
\mathbf{0}_{q} & \mathbf{I}_{q} \\
-\mathbf{I}_{q} & \mathbf{0}_{q}
\end{array}\right)=-\mathbf{I}_{2 q}
$$

Corollary 3.6. For each integer $j \in\{0,1, \ldots, \tau\}$ and point $u \in \mathbb{C} \backslash\left(\mathbb{P}_{\alpha, j} \cup \mathbb{Z}_{\alpha, j}\right)$, the matrices $\boldsymbol{\Xi}_{j}(u), \boldsymbol{\Theta}_{j}(u)$ are nonsingular and

$$
\begin{gathered}
\left(\boldsymbol{\Xi}_{j}(u)\right)^{-1}=\frac{\left(1-\overline{\alpha_{j}} u\right)^{2}}{2 \eta_{j}\left(1-\left|\alpha_{j}\right|^{2}\right) B_{j}(u)}\left(\begin{array}{cc}
X_{j}^{[\alpha, j]}(u) & -b_{\alpha_{j}}(u) Y_{j}(u) \\
-\left(X_{j}^{\#}\right)^{[\alpha, j]}(u) & -b_{\alpha_{j}}(u) Y_{j}^{\#}(u)
\end{array}\right), \\
\left(\boldsymbol{\Theta}_{j}(u)\right)^{-1}=\frac{\left(1-\overline{\alpha_{j}} u\right)^{2}}{2 \eta_{j}\left(1-\left|\alpha_{j}\right|^{2}\right) B_{j}(u)}\left(\begin{array}{cc}
Y_{j}^{[\alpha, j]}(u) & -\left(Y_{j}^{\#}\right)^{[\alpha, j]}(u) \\
-b_{\alpha_{j}}(u) X_{j}(u) & -b_{\alpha_{j}}(u) X_{j}^{\#}(u)
\end{array}\right) .
\end{gathered}
$$

Proposition 3.5 and Corollary 3.6 imply by considering the $q \times q$ block entries of $\boldsymbol{\Theta}_{j}$ and $\boldsymbol{\Xi}_{j}, j \in\{0,1, \ldots, \tau\}$, according to (2.12) the identities below.

Corollary 3.7. For each integer $j \in\{0,1, \ldots, \tau\}$ and point $u \in \mathbb{C} \backslash \mathbb{P}_{\alpha, j}$,

$$
\begin{gathered}
X_{j}(u) Y_{j}^{\#}(u)=X_{j}^{\#}(u) Y_{j}(u), \quad\left(Y_{j}^{\#}\right)^{[\alpha, j]}(u) X_{j}^{[\alpha, j]}(u)=Y_{j}^{[\alpha, j]}(u)\left(X_{j}^{\#}\right)^{[\alpha, j]}(u), \\
X_{j}^{[\alpha, j]}(u) X_{j}(u)=Y_{j}(u) Y_{j}^{[\alpha, j]}(u), \quad\left(X_{j}^{\#}\right)^{[\alpha, j]}(u) X_{j}^{\#}(u)=Y_{j}^{\#}(u)\left(Y_{j}^{\#}\right)^{[\alpha, j]}(u), \\
b_{\alpha_{j}}(u)\left(X_{j}(u)\left(X_{j}^{\#}\right)^{[\alpha, j]}(u)+X_{j}^{\#}(u) X_{j}^{[\alpha, j]}(u)\right)=-2 \eta_{j} \frac{1-\left|\alpha_{j}\right|^{2}}{\left(1-\overline{\alpha_{j}} u\right)^{2}} B_{j}(u) \mathbf{I}_{q}, \\
b_{\alpha_{j}}(u)\left(\left(Y_{j}^{\#}\right)^{[\alpha, j]}(u) Y_{j}(u)+Y_{j}^{[\alpha, j]}(u) Y_{j}^{\#}(u)\right)=-2 \eta_{j} \frac{1-\left|\alpha_{j}\right|^{2}}{\left(1-\overline{\alpha_{j}} u\right)^{2}} B_{j}(u) \mathbf{I}_{q}, \\
b_{\alpha_{j}}(u)\left(\left(X_{j}^{\#}\right)^{[\alpha, j]}(u) X_{j}(u)+Y_{j}^{\#}(u) Y_{j}^{[\alpha, j]}(u)\right)=-2 \eta_{j} \frac{1-\left|\alpha_{j}\right|^{2}}{\left(1-\overline{\alpha_{j}} u\right)^{2}} B_{j}(u) \mathbf{I}_{q}, \\
b_{\alpha_{j}}(u)\left(X_{j}^{[\alpha, j]}(u) X_{j}^{\#}(u)+Y_{j}(u)\left(Y_{j}^{\#}\right)^{[\alpha, j]}(u)\right)=-2 \eta_{j} \frac{1-\left|\alpha_{j}\right|^{2}}{\left(1-\overline{\alpha_{j}} u\right)^{2}} B_{j}(u) \mathbf{I}_{q} .
\end{gathered}
$$


Remark 3.8. For all integers $\ell \in\{1,2, \ldots, \tau\}$ and $j \in\{0,1, \ldots, \ell-1\}$, Corollary 3.7 implies

$$
\begin{array}{ll}
X_{\ell}\left(\alpha_{j}\right)\left(X_{\ell}^{\#}\right)^{[\alpha, \ell]}\left(\alpha_{j}\right)=-X_{\ell}^{\#}\left(\alpha_{j}\right) X_{\ell}^{[\alpha, \ell]}\left(\alpha_{j}\right), & \left(Y_{\ell}^{\#}\right)^{[\alpha, \ell]}\left(\alpha_{j}\right) Y_{\ell}\left(\alpha_{j}\right)=-Y_{\ell}^{[\alpha, \ell]}\left(\alpha_{j}\right) Y_{\ell}^{\#}\left(\alpha_{j}\right), \\
\left(X_{\ell}^{\#}\right)^{[\alpha, \ell]}\left(\alpha_{j}\right) X_{\ell}\left(\alpha_{j}\right)=-Y_{\ell}^{\#}\left(\alpha_{j}\right) Y_{\ell}^{[\alpha, \ell]}\left(\alpha_{j}\right), & X_{\ell}^{[\alpha, \ell]}\left(\alpha_{j}\right) X_{\ell}^{\#}\left(\alpha_{j}\right)=-Y_{\ell}\left(\alpha_{j}\right)\left(Y_{\ell}^{\#}\right)^{[\alpha, \ell]}\left(\alpha_{j}\right),
\end{array}
$$

since the points $\alpha_{0}, \alpha_{1}, \ldots, \alpha_{\ell-1}$ are the zeros of the function $B_{\ell-1}$ (cf. (2.2)).

Proposition 3.9. For all integers $\ell \in\{1,2, \ldots, \tau\}$ and $j \in\{0,1, \ldots, \ell-1\}$,

$$
\begin{aligned}
\left(X_{\ell}^{\#}\right)^{[\alpha, \ell]}\left(\alpha_{j}\right)\left(X_{\ell}^{[\alpha, \ell]}\left(\alpha_{j}\right)\right)^{-1} & =\left(X_{\ell-1}^{\#}\right)^{[\alpha, \ell-1]}\left(\alpha_{j}\right)\left(X_{\ell-1}^{[\alpha, \ell-1]}\left(\alpha_{j}\right)\right)^{-1} \\
& =\left(Y_{\ell-1}^{[\alpha, \ell-1]}\left(\alpha_{j}\right)\right)^{-1}\left(Y_{\ell-1}^{\#}\right)^{[\alpha, \ell-1]}\left(\alpha_{j}\right) \\
& =\left(Y_{\ell}^{[\alpha, \ell]}\left(\alpha_{j}\right)\right)^{-1}\left(Y_{\ell}^{\#}\right)^{[\alpha, \ell]}\left(\alpha_{j}\right) .
\end{aligned}
$$

Proof. Let $\ell \in\{1,2, \ldots, \tau\}$ and $j \in\{0,1, \ldots, \ell-1\}$. From Corollary 3.2 it follows that $\operatorname{det} X_{\ell}^{[\alpha, \ell]}\left(\alpha_{j}\right) \neq 0, \operatorname{det} X_{\ell-1}^{[\alpha, \ell-1]}\left(\alpha_{j}\right) \neq 0, \operatorname{det} Y_{\ell}^{[\alpha, \ell]}\left(\alpha_{j}\right) \neq 0, \operatorname{det} Y_{\ell-1}^{[\alpha, \ell-1]}\left(\alpha_{j}\right) \neq 0$, and $\operatorname{det}\left(Y_{\ell-1}^{\#}\right)^{[\alpha, \ell-1]}\left(\alpha_{j}\right) \neq 0$. Hence, Corollary 3.7 provides the equalities

$$
\begin{gathered}
\left(X_{\ell}^{\#}\right)^{[\alpha, \ell]}\left(\alpha_{j}\right)\left(X_{\ell}^{[\alpha, \ell]}\left(\alpha_{j}\right)\right)^{-1}=\left(Y_{\ell}^{[\alpha, \ell]}\left(\alpha_{j}\right)\right)^{-1}\left(Y_{\ell}^{\#}\right)^{[\alpha, \ell]}\left(\alpha_{j}\right), \\
\left(X_{\ell-1}^{\#}\right)^{[\alpha, \ell-1]}\left(\alpha_{j}\right)\left(X_{\ell-1}^{[\alpha, \ell-1]}\left(\alpha_{j}\right)\right)^{-1}=\left(Y_{\ell-1}^{[\alpha, \ell-1]}\left(\alpha_{j}\right)\right)^{-1}\left(Y_{\ell-1}^{\#}\right)^{[\alpha, \ell-1]}\left(\alpha_{j}\right) .
\end{gathered}
$$

Furthermore, if $j=\ell-1$, then Corollary 3.3 yields

$$
\left(X_{\ell}^{\#}\right)^{[\alpha, \ell]}\left(\alpha_{j}\right)\left(X_{\ell}^{[\alpha, \ell]}\left(\alpha_{j}\right)\right)^{-1}=\left(X_{\ell-1}^{\#}\right)^{[\alpha, \ell-1]}\left(\alpha_{j}\right)\left(X_{\ell-1}^{[\alpha, \ell-1]}\left(\alpha_{j}\right)\right)^{-1}
$$

and if $j \neq \ell-1$, then from (2.13), Corollary 3.7, and Remark 3.8, one can get

$$
\begin{aligned}
\left(Y_{\ell-1}\right)^{[\alpha, \ell-1]}\left(\alpha_{j}\right)\left(X_{\ell}^{\#}\right)^{[\alpha, \ell]}\left(\alpha_{j}\right)\left(X_{\ell}^{[\alpha, \ell]}\left(\alpha_{j}\right)\right)^{-1}\left(\left(Y_{\ell-1}^{\#}\right)^{[\alpha, \ell-1]}\left(\alpha_{j}\right)\right)^{-1} \\
=\left(Y_{\ell-1}\right)^{[\alpha, \ell-1]}\left(\alpha_{j}\right)\left(\left(X_{\ell-1}^{\#}\right)^{[\alpha, \ell-1]}\left(\alpha_{j}\right)-b_{\alpha_{\ell-1}}\left(\alpha_{j}\right) Y_{\ell-1}^{\#}\left(\alpha_{j}\right) \mathbf{E}_{\ell}^{*}\right) \\
\quad \times\left(\left(X_{\ell-1}\right)^{[\alpha, \ell-1]}\left(\alpha_{j}\right)+b_{\alpha_{\ell-1}} Y_{\ell-1}\left(\alpha_{j}\right) \mathbf{E}_{\ell}^{*}\right)^{-1}\left(\left(Y_{\ell-1}^{\#}\right)^{[\alpha, \ell-1]}\left(\alpha_{j}\right)\right)^{-1} \\
=\left(\left(Y_{\ell-1}\right)^{[\alpha, \ell-1]}\left(\alpha_{j}\right)\left(X_{\ell-1}^{\#}\right)^{[\alpha, \ell-1]}\left(\alpha_{j}\right)-b_{\alpha_{\ell-1}}\left(\alpha_{j}\right)\left(Y_{\ell-1}\right)^{[\alpha, \ell-1]}\left(\alpha_{j}\right) Y_{\ell-1}^{\#}\left(\alpha_{j}\right) \mathbf{E}_{\ell}^{*}\right) \\
\quad \times\left(\left(Y_{\ell-1}\right)^{[\alpha, \ell-1]}\left(\alpha_{j}\right)\left(X_{\ell-1}^{\#}\right)^{[\alpha, \ell-1]}\left(\alpha_{j}\right)-b_{\alpha_{\ell-1}}\left(\alpha_{j}\right)\left(Y_{\ell-1}\right)^{[\alpha, \ell-1]}\left(\alpha_{j}\right) Y_{\ell-1}^{\#}\left(\alpha_{j}\right) \mathbf{E}_{\ell}^{*}\right)^{-1}=\mathbf{I}_{q},
\end{aligned}
$$

that is, $\left(X_{\ell}^{\#}\right)^{[\alpha, \ell]}\left(\alpha_{j}\right)\left(X_{\ell}^{[\alpha, \ell]}\left(\alpha_{j}\right)\right)^{-1}=\left(\left(Y_{\ell-1}\right)^{[\alpha, \ell-1]}\left(\alpha_{j}\right)\right)^{-1}\left(Y_{\ell-1}^{\#}\right)^{[\alpha, \ell-1]}\left(\alpha_{j}\right)$, which completes the proof.

Proposition 3.9 implies in view of (2.4), (2.6), and (2.1) the following. 
Corollary 3.10. For each integer $j \in\{0,1, \ldots, \tau\}$,

$$
\left(X_{j}^{\#}\right)^{[\alpha, j]}\left(\alpha_{0}\right)\left(X_{j}^{[\alpha, j]}\left(\alpha_{0}\right)\right)^{-1}=\left(\mathbf{X}_{0}^{*} \mathbf{X}_{0}\right)^{-1}=\left(\mathbf{Y}_{0} \mathbf{Y}_{0}^{*}\right)^{-1}=\left(Y_{j}^{[\alpha, j]}\left(\alpha_{0}\right)\right)^{-1}\left(Y_{j}^{\#}\right)^{[\alpha, j]}\left(\alpha_{0}\right) .
$$

Proposition 3.11. For each integer $\ell \in\{1,2, \ldots, \tau\}$, the $2 q \times 2 q$ matrices

$$
\begin{aligned}
& \frac{1-\left|\alpha_{\ell}\right|^{2}}{\left|1-\overline{\alpha_{\ell}} u\right|^{2}}\left(\left(\boldsymbol{\Theta}_{\ell}(u)\right)^{-1}\right)^{*} \mathbf{j}_{q q}\left(\boldsymbol{\Theta}_{\ell}(u)\right)^{-1}-\frac{1-\left|\alpha_{\ell-1}\right|^{2}}{\left|1-\overline{\alpha_{\ell-1}} u\right|^{2}}\left(\left(\boldsymbol{\Theta}_{\ell-1}(u)\right)^{-1}\right)^{*} \mathbf{j}_{q q}\left(\boldsymbol{\Theta}_{\ell-1}(u)\right)^{-1}, \\
& \frac{1-\left|\alpha_{\ell}\right|^{2}}{\left|1-\overline{\alpha_{\ell}} u\right|^{2}}\left(\boldsymbol{\Xi}_{\ell}(u)\right)^{-1} \mathbf{j}_{q q}\left(\left(\boldsymbol{\Xi}_{\ell}(u)\right)^{-1}\right)^{*}-\frac{1-\left|\alpha_{\ell-1}\right|^{2}}{\left|1-\overline{\alpha_{\ell-1}} u\right|^{2}}\left(\boldsymbol{\Xi}_{\ell-1}(u)\right)^{-1} \mathbf{j}_{q q}\left(\left(\boldsymbol{\Xi}_{\ell-1}(u)\right)^{-1}\right)^{*}
\end{aligned}
$$

are nonnegative Hermitian if $u \in \mathbb{D} \backslash \mathbb{Z}_{\alpha, \ell}$ and if $u \in \mathbb{T}$, then

$$
\begin{aligned}
& \frac{1-\left|\alpha_{\ell}\right|^{2}}{\left|1-\overline{\alpha_{\ell}} u\right|^{2}}\left(\left(\boldsymbol{\Theta}_{\ell}(u)\right)^{-1}\right)^{*} \mathbf{j}_{q q}\left(\boldsymbol{\Theta}_{\ell}(u)\right)^{-1}=\frac{1-\left|\alpha_{\ell-1}\right|^{2}}{\left|1-\overline{\alpha_{\ell-1}} u\right|^{2}}\left(\left(\boldsymbol{\Theta}_{\ell-1}(u)\right)^{-1}\right)^{*} \mathbf{j}_{q q}\left(\boldsymbol{\Theta}_{\ell-1}(u)\right)^{-1}, \\
& \frac{1-\left|\alpha_{\ell}\right|^{2}}{\left|1-\overline{\alpha_{\ell}} u\right|^{2}}\left(\boldsymbol{\Xi}_{\ell}(u)\right)^{-1} \mathbf{j}_{q q}\left(\left(\boldsymbol{\Xi}_{\ell}(u)\right)^{-1}\right)^{*}=\frac{1-\left|\alpha_{\ell-1}\right|^{2}}{\left|1-\overline{\alpha_{\ell-1}} u\right|^{2}}\left(\boldsymbol{\Xi}_{\ell-1}(u)\right)^{-1} \mathbf{j}_{q q}\left(\left(\boldsymbol{\Xi}_{\ell-1}(u)\right)^{-1}\right)^{*}
\end{aligned}
$$

where $\boldsymbol{\Theta}_{\ell}(u), \boldsymbol{\Theta}_{\ell-1}(u), \boldsymbol{\Xi}_{\ell}(u)$, and $\boldsymbol{\Xi}_{\ell-1}(u)$ are defined as in (2.12).

Proof. Let $\ell \in\{1,2, \ldots, \tau\}$ and $u \in(\mathbb{D} \cup \mathbb{T}) \backslash \mathbb{Z}_{\alpha, \ell}$. We prove only the assertion with respect to $\Theta_{\ell}(u)$. Similarly, one can verify the others by using the same arguments. From Corollary 3.6 we know that the matrix $\boldsymbol{\Theta}_{\ell-1}(u)$ is nonsingular. Consequently,

$$
\sqrt{\frac{1-\left|\alpha_{\ell-1}\right|^{2}}{1-\left|\alpha_{\ell}\right|^{2}}} \frac{1-\overline{\alpha_{\ell}} u}{1-\overline{\alpha_{\ell-1}} u}\left(\boldsymbol{\Theta}_{\ell-1}(u)\right)^{-1} \boldsymbol{\Theta}_{\ell}(u)=\mathbf{H}_{\mathbf{E}_{\ell}^{*}}\left(\begin{array}{cc}
b_{\alpha_{\ell}}(u) \mathbf{I}_{q} & \mathbf{0}_{q} \\
\mathbf{0}_{q} & \eta_{\ell} \overline{\eta_{\ell-1}} \mathbf{I}_{q}
\end{array}\right)
$$

follows from (2.13). Hence (cf. the proof of Theorem 3.1), the matrix

$$
\mathbf{A}:=\sqrt{\frac{1-\left|\alpha_{\ell-1}\right|^{2}}{1-\left|\alpha_{\ell}\right|^{2}}} \frac{1-\overline{\alpha_{\ell}} u}{1-\overline{\alpha_{\ell-1}} u}\left(\boldsymbol{\Theta}_{\ell-1}(u)\right)^{-1} \boldsymbol{\Theta}_{\ell}(u)
$$

is $\mathbf{j}_{q q}$-contractive if $u \in \mathbb{D} \backslash \mathbb{Z}_{\alpha, \ell}$ and $\mathbf{j}_{q q}$-unitary if $u \in \mathbb{T}$. Therefore, the identity

$$
\begin{gathered}
\frac{1-\left|\alpha_{\ell}\right|^{2}}{\left|1-\overline{\alpha_{\ell}} u\right|^{2}}\left(\left(\boldsymbol{\Theta}_{\ell}(u)\right)^{-1}\right)^{*} \mathbf{j}_{q q}\left(\boldsymbol{\Theta}_{\ell}(u)\right)^{-1}-\frac{1-\left|\alpha_{\ell-1}\right|^{2}}{\left|1-\overline{\alpha_{\ell-1}} u\right|^{2}}\left(\left(\boldsymbol{\Theta}_{\ell-1}(u)\right)^{-1}\right)^{*} \mathbf{j}_{q q}\left(\boldsymbol{\Theta}_{\ell-1}(u)\right)^{-1} \\
=\frac{1-\left|\alpha_{\ell}\right|^{2}}{\left|1-\overline{\alpha_{\ell}} u\right|^{2}}\left(\left(\boldsymbol{\Theta}_{\ell}(u)\right)^{-1}\right)^{*}\left(\mathbf{j}_{q q}-\mathbf{A}^{*} \mathbf{j}_{q q} \mathbf{A}\right)\left(\boldsymbol{\Theta}_{\ell}(u)\right)^{-1}
\end{gathered}
$$

implies immediately the assertion referring to $\boldsymbol{\Theta}_{\ell}(u)$. 


\section{Christoffel-Darboux formulae}

We show in the present section that, similar as for orthogonal rational matrix-valued functions (cf. [25, Section 5]), any Szegö pair of rational matrix-valued functions along with its dual Szegö pair satisfies some Christoffel-Darboux formulae. Here $\left[\left(X_{j}\right)_{j=0}^{\tau}\right.$, $\left.\left(Y_{j}\right)_{j=0}^{\tau}\right]$ denotes again the Szegö pair of rational matrix-valued functions generated by $\left[\left(\alpha_{j}\right)_{j=0}^{\tau} ;\left(\mathbf{E}_{\ell}\right)_{\ell=1}^{\tau} ; \mathbf{X}_{0}, \mathbf{Y}_{0}\right]$ and $\left[\left(X_{j}^{\#}\right)_{j=0}^{\tau},\left(Y_{j}^{\#}\right)_{j=0}^{\tau}\right]$ stands for its dual Szegö pair, where $\left(\alpha_{j}\right)_{j=0}^{\tau}$ is a sequence of points belonging to $\mathbb{D}$, where $\left(\mathbf{E}_{\ell}\right)_{\ell=1}^{\tau}$ is a sequence of strictly contractive $q \times q$ matrices, and where $\mathbf{X}_{0}, \mathbf{Y}_{0}$ are some nonsingular complex $q \times q$ matrices fulfilling $\mathbf{X}_{0}^{*} \mathbf{X}_{0}=\mathbf{Y}_{0} \mathbf{Y}_{0}^{*}$. For technical reasons, if $\alpha_{j} \in \mathbb{D}$ and if $u, v \in \mathbb{C} \backslash\left\{1 / \overline{\alpha_{j}}\right\}$, we also use the notation

$$
k_{\alpha_{j}}(u, v):=1-b_{\alpha_{j}}(u) \overline{b_{\alpha_{j}}(v)}=\frac{\left(1-\left|\alpha_{j}\right|^{2}\right)(1-u \bar{v})}{\left(1-u \overline{\alpha_{j}}\right)\left(1-\alpha_{j} \bar{v}\right)} .
$$

At first, we remark the following identities with respect to $\left[\left(X_{j}\right)_{j=0}^{\tau},\left(Y_{j}\right)_{j=0}^{\tau}\right]$ and $\left[\left(X_{j}^{\#}\right)_{j=0}^{\tau}\right.$, $\left.\left(Y_{j}^{\#}\right)_{j=0}^{\tau}\right]$.

Lemma 4.1. For each integer $\ell \in\{1,2, \ldots, \tau\}$ and points $u, v \in \mathbb{C} \backslash \mathbb{P}_{\alpha, \ell}$,

$$
\begin{aligned}
& k_{\alpha_{\ell-1}}(u, v)\left(\left(Y_{\ell}^{[\alpha, \ell]}(v)\right)^{*} Y_{\ell}^{[\alpha, \ell]}(u)-\left(X_{\ell}(v)\right)^{*} X_{\ell}(u)\right) \\
& =k_{\alpha_{\ell}}(u, v)\left(\left(Y_{\ell-1}^{[\alpha, \ell-1]}(v)\right)^{*} Y_{\ell-1}^{[\alpha, \ell-1]}(u)-\overline{b_{\alpha_{\ell-1}}(v)} b_{\alpha_{\ell-1}}(u)\left(X_{\ell-1}(v)\right)^{*} X_{\ell-1}(u)\right), \\
& k_{\alpha_{\ell-1}}(u, v)\left(X_{\ell}^{[\alpha, \ell]}(u)\left(X_{\ell}^{[\alpha, \ell]}(v)\right)^{*}-Y_{\ell}(u)\left(Y_{\ell}(v)\right)^{*}\right) \\
& =k_{\alpha_{\ell}}(u, v)\left(X_{\ell-1}^{[\alpha, \ell-1]}(u)\left(X_{\ell-1}^{[\alpha, \ell-1]}(v)\right)^{*}-b_{\alpha_{\ell-1}}(u) \overline{b_{\alpha_{\ell-1}}(v)} Y_{\ell-1}(u)\left(Y_{\ell-1}(v)\right)^{*}\right), \\
& k_{\alpha_{\ell-1}}(u, v)\left(\left(\left(Y_{\ell}^{\#}\right)^{[\alpha, \ell]}(v)\right)^{*} Y_{\ell}^{[\alpha, \ell]}(u)+\left(X_{\ell}^{\#}(v)\right)^{*} X_{\ell}(u)\right) \\
& =k_{\alpha_{\ell}}(u, v)\left(\left(\left(Y_{\ell-1}^{\#}\right)^{[\alpha, \ell-1]}(v)\right)^{*} Y_{\ell-1}^{[\alpha, \ell-1]}(u)+\overline{b_{\alpha_{\ell-1}}(v)} b_{\alpha_{\ell-1}}(u)\left(X_{\ell-1}^{\#}(v)\right)^{*} X_{\ell-1}(u)\right), \\
& k_{\alpha_{\ell-1}}(u, v)\left(X_{\ell}^{[\alpha, \ell]}(u)\left(\left(X_{\ell}^{\#}\right)^{[\alpha, \ell]}(v)\right)^{*}+Y_{\ell}(u)\left(Y_{\ell}^{\#}(v)\right)^{*}\right) \\
& =k_{\alpha_{\ell}}(u, v)\left(X_{\ell-1}^{[\alpha, \ell-1]}(u)\left(\left(X_{\ell-1}^{\#}\right)^{[\alpha, \ell-1]}(v)\right)^{*}+b_{\alpha_{\ell-1}}(u) \overline{b_{\alpha_{\ell-1}}(v)} Y_{\ell-1}(u)\left(Y_{\ell-1}^{\#}(v)\right)^{*}\right) .
\end{aligned}
$$

Proof. Let $\ell \in\{1,2, \ldots, \tau\}$ and let $u, v \in \mathbb{C} \backslash \mathbb{P}_{\alpha, \ell}$. Considering the second $q \times q$ block row of $\boldsymbol{\Theta}_{\ell}(u)$ and the first $q \times q$ block column of $\left(\boldsymbol{\Theta}_{\ell}(v)\right)^{*}$, from (2.13) and (3.5) (note also 
[11, Theorem 1.3.3]) one can see

$$
\begin{aligned}
& -\frac{1-\left|\alpha_{\ell-1}\right|^{2}}{1-\left|\alpha_{\ell}\right|^{2}} \frac{1-\overline{\alpha_{\ell}} u}{1-\overline{\alpha_{\ell-1}} u} \frac{1-\alpha_{\ell} \bar{v}}{1-\alpha_{\ell-1} \bar{v}}\left(X_{\ell}^{[\alpha, \ell]}(u)\left(\left(X_{\ell}^{\#}\right)^{[\alpha, \ell]}(v)\right)^{*}+Y_{\ell}(u)\left(Y_{\ell}^{\#}(v)\right)^{*}\right) \\
& =\frac{1-\left|\alpha_{\ell-1}\right|^{2}}{1-\left|\alpha_{\ell}\right|^{2}} \frac{1-\overline{\alpha_{\ell}} u}{1-\overline{\alpha_{\ell-1}} u} \frac{1-\alpha_{\ell} \bar{v}}{1-\alpha_{\ell-1} \bar{v}}\left(Y_{\ell}(u) \overline{\eta_{\ell}} \eta_{\ell-1} X_{\ell}^{[\alpha, \ell]}(u)\right) \mathbf{j}_{q q}\left(\begin{array}{c}
-\left(Y_{\ell}^{\#}(v)\right)^{*} \\
\eta_{\ell} \overline{\eta_{\ell-1}}\left(\left(X_{\ell}^{\#}\right)^{[\alpha, \ell]}(v)\right)^{*}
\end{array}\right) \\
& =\left(b_{\alpha_{\ell-1}}(u) Y_{\ell-1}(u) X_{\ell-1}^{[\alpha, \ell-1]}(u)\right) \mathbf{H}_{\mathbf{E}_{\ell}^{*}} \mathbf{j}_{q q}\left(\mathbf{H}_{\mathbf{E}_{\ell}^{*}}\right)^{*}\left(\begin{array}{c}
-\overline{b_{\alpha_{\ell-1}}(v)}\left(Y_{\ell-1}^{\#}(v)\right)^{*} \\
\left(\left(X_{\ell-1}^{\#}\right)^{[\alpha, \ell-1]}(v)\right)^{*}
\end{array}\right) \\
& =\left(b_{\alpha_{\ell-1}}(u) Y_{\ell-1}(u) X_{\ell-1}^{[\alpha, \ell-1]}(u)\right)\left(\begin{array}{l}
-\overline{b_{\alpha_{\ell-1}}(v)}\left(Y_{\ell-1}^{\#}(v)\right)^{*} \\
-\left(\left(X_{\ell-1}^{\#}\right)^{[\alpha, \ell-1]}(v)\right)^{*}
\end{array}\right) \\
& =-X_{\ell-1}^{[\alpha, \ell-1]}(u)\left(\left(X_{\ell-1}^{\#}\right)^{[\alpha, \ell-1]}(v)\right)^{*}-b_{\alpha_{\ell-1}}(u) \overline{b_{\alpha_{\ell-1}}(v)} Y_{\ell-1}(u)\left(Y_{\ell-1}^{\#}(v)\right)^{*} \text {. }
\end{aligned}
$$

Hence, (4.1) yields the fourth identity. Similarly, observing further combinations of $q \times q$ block rows and $q \times q$ block columns of the matrix-valued function $\boldsymbol{\Theta}_{\ell}$ or $\boldsymbol{\Xi}_{\ell}$, in view of (2.13), (3.5), and (4.1), one can obtain the other equalities.

Theorem 4.2. For each integer $j \in\{0,1, \ldots, \tau\}$ and points $u, v \in \mathbb{C} \backslash \mathbb{P}_{\alpha, j}$, the following Christoffel-Darboux formulae hold:

$$
\begin{aligned}
& \left(1-\overline{b_{\alpha_{j}}(v)} b_{\alpha_{j}}(u)\right) \sum_{k=0}^{j}\left(X_{k}(v)\right)^{*} X_{k}(u) \\
& =\left(Y_{j}^{[\alpha, j]}(v)\right)^{*} Y_{j}^{[\alpha, j]}(u)-\overline{b_{\alpha_{j}}(v)} b_{\alpha_{j}}(u)\left(X_{j}(v)\right)^{*} X_{j}(u), \\
& \left(1-b_{\alpha_{j}}(u) \overline{b_{\alpha_{j}}(v)}\right) \sum_{k=0}^{j} Y_{k}(u)\left(Y_{k}(v)\right)^{*} \\
& =X_{j}^{[\alpha, j]}(u)\left(X_{j}^{[\alpha, j]}(v)\right)^{*}-b_{\alpha_{j}}(u) \overline{b_{\alpha_{j}}(v)} Y_{j}(u)\left(Y_{j}(v)\right)^{*}, \\
& \left(1-\overline{b_{\alpha_{j}}(v)} b_{\alpha_{j}}(u)\right) \sum_{k=0}^{j}\left(X_{k}^{\#}(v)\right)^{*} X_{k}(u) \\
& =\frac{2\left(1-\left|\alpha_{j}\right|^{2}\right)}{\left(1-\alpha_{j} \bar{v}\right)\left(1-u \overline{\alpha_{j}}\right)} \mathbf{I}_{q}-\left(\left(Y_{j}^{\#}\right)^{[\alpha, j]}(v)\right)^{*} Y_{j}^{[\alpha, j]}(u)-\overline{b_{\alpha_{j}}(v)} b_{\alpha_{j}}(u)\left(X_{j}^{\#}(v)\right)^{*} X_{j}(u), \\
& \left(1-b_{\alpha_{j}}(u) \overline{b_{\alpha_{j}}(v)}\right) \sum_{k=0}^{j} Y_{k}(u)\left(Y_{k}^{\#}(v)\right)^{*} \\
& =\frac{2\left(1-\left|\alpha_{j}\right|^{2}\right)}{\left(1-u \overline{\alpha_{j}}\right)\left(1-\alpha_{j} \bar{v}\right)} \mathbf{I}_{q}-X_{j}^{[\alpha, j]}(u)\left(\left(X_{j}^{\#}\right)^{[\alpha, j]}(v)\right)^{*}-b_{\alpha_{j}}(u) \overline{b_{\alpha_{j}}(v)} Y_{j}(u)\left(Y_{j}^{\#}(v)\right)^{*} \text {. }
\end{aligned}
$$


Proof. Let $u, v \in \mathbb{C} \backslash \mathbb{P}_{\alpha, 0}$. According to (2.1), (2.4), and (2.6) we have

$$
\begin{aligned}
X_{0}^{[\alpha, 0]}(u)\left(\left(X_{0}^{\#}\right)^{[\alpha, 0]}(v)\right)^{*} & =\left(\frac{-\eta_{0} \sqrt{1-\left|\alpha_{0}\right|^{2}}}{1-\overline{\alpha_{0}} u} \mathbf{X}_{0}^{*}\right)\left(\frac{-\eta_{0} \sqrt{1-\left|\alpha_{0}\right|^{2}}}{1-\overline{\alpha_{0}} v} \mathbf{X}_{0}^{-1}\right)^{*} \\
& =\frac{1-\left|\alpha_{0}\right|^{2}}{\left(1-u \overline{\alpha_{0}}\right)\left(1-\alpha_{0} \bar{v}\right)} \mathbf{I}_{q}, \\
Y_{0}(u)\left(Y_{0}^{\#}(v)\right)^{*} & =\left(\frac{\sqrt{1-\left|\alpha_{0}\right|^{2}}}{1-\overline{\alpha_{0}} u} \mathbf{Y}_{0}\right)\left(\frac{\sqrt{1-\left|\alpha_{0}\right|^{2}}}{1-\overline{\alpha_{0}} v}\left(\mathbf{Y}_{0}^{-1}\right)^{*}\right)^{*} \\
& =\frac{1-\left|\alpha_{0}\right|^{2}}{\left(1-u \overline{\alpha_{0}}\right)\left(1-\alpha_{0} \bar{v}\right)} \mathbf{I}_{q} .
\end{aligned}
$$

Hence, the fourth identity is obviously fulfilled for $j=0$. Now we assume that for each integer $\ell \in\{1,2, \ldots, \tau\}$ and points $u, v \in \mathbb{C} \backslash \mathbb{P}_{\alpha, \ell}$ the fourth formula with $j:=\ell-1$ is already proved. Thus, the fourth equality in Lemma 4.1 and (4.1) imply

$$
\begin{aligned}
(1- & \left.b_{\alpha_{\ell}}(u) \overline{b_{\alpha_{\ell}}(v)}\right) \sum_{k=0}^{\ell} Y_{k}(u)\left(Y_{k}^{\#}(v)\right)^{*} \\
= & \frac{k_{\alpha_{\ell}}(u, v)}{k_{\alpha_{\ell-1}}(u, v)}\left(1-b_{\alpha_{\ell-1}}(u) \overline{b_{\alpha_{\ell-1}}(v)}\right) \sum_{k=0}^{\ell-1} Y_{k}(u)\left(Y_{k}^{\#}(v)\right)^{*}+k_{\alpha_{\ell}}(u, v) Y_{\ell}(u)\left(Y_{\ell}^{\#}(v)\right)^{*} \\
= & -\frac{k_{\alpha_{\ell}}(u, v)}{k_{\alpha_{\ell-1}}(u, v)}\left(X_{\ell-1}^{[\alpha, \ell-1]}(u)\left(\left(X_{\ell-1}^{\#}\right)^{[\alpha, \ell-1]}(v)\right)^{*}+b_{\alpha_{\ell-1}}(u) \overline{b_{\alpha_{\ell-1}}(v)} Y_{\ell-1}(u)\left(Y_{\ell-1}^{\#}(v)\right)^{*}\right) \\
& +\frac{2 k_{\alpha_{\ell}}(u, v)\left(1-\left|\alpha_{\ell-1}\right|^{2}\right)}{k_{\alpha_{\ell-1}}(u, v)\left(1-u \overline{\alpha_{\ell-1}}\right)\left(1-\alpha_{\ell-1} \bar{v}\right)} \mathbf{I}_{q}+Y_{\ell}(u)\left(Y_{\ell}^{\#}(v)\right)^{*}-b_{\alpha_{\ell}}(u) \overline{b_{\alpha_{\ell}}(v)} Y_{\ell}(u)\left(Y_{\ell}^{\#}(v)\right)^{*} \\
= & -X_{\ell}^{[\alpha, \ell]}(u)\left(\left(X_{\ell}^{\#}\right)^{[\alpha, \ell]}(v)\right)^{*}-Y_{\ell}(u)\left(Y_{\ell}^{\#}(v)\right)^{*}+\frac{2\left(1-\left|\alpha_{\ell}\right|^{2}\right)}{\left(1-u \overline{\alpha_{\ell}}\right)\left(1-\alpha_{\ell} \bar{v}\right)} \mathbf{I}_{q} \\
& +Y_{\ell}(u)\left(Y_{\ell}^{\#}(v)\right)^{*}-b_{\alpha_{\ell}}(u) \overline{b_{\alpha_{\ell}}(v)} Y_{\ell}(u)\left(Y_{\ell}^{\#}(v)\right)^{*} \\
= & \frac{2\left(1-\left|\alpha_{\ell}\right|^{2}\right)}{\left(1-u \overline{\alpha_{\ell}}\right)\left(1-\alpha_{\ell} \bar{v}\right)} \mathbf{I}_{q}-X_{\ell}^{[\alpha, \ell]}(u)\left(\left(X_{\ell}^{\#}\right)^{[\alpha, \ell]}(v)\right)^{*}-b_{\alpha_{\ell}}(u) \overline{b_{\alpha_{\ell}}(v)} Y_{\ell}(u)\left(Y_{\ell}^{\#}(v)\right)^{*}
\end{aligned}
$$

firstly for $u, v \in \mathbb{C} \backslash \mathbb{P}_{\alpha, \ell}$ satisfying $u \bar{v} \neq 1$. Applying a continuity argument one can get that this identity is actually fulfilled for all $u, v \in \mathbb{C} \backslash \mathbb{P}_{\alpha, \ell}$. Consequently, for each integer $j \in\{0,1, \ldots, \tau\}$ and points $u, v \in \mathbb{C} \backslash \mathbb{P}_{\alpha, j}$ the fourth formula is inductively shown. Similarly, the first, second, and third formulae can be verified by using (2.1), (2.4), (2.6), Lemma 4.1, and (4.1). 
Observe that the first and second Christoffel-Darboux formulae in Theorem 4.2 coincide with the identities for orthogonal rational matrix-valued functions proved in [25, Section 5] (see also [27, Theorem 3.10]). In view of Remark 2.1, these relations yield for each integer $j \in\{0,1, \ldots, \tau\}$ and points $u, v \in \mathbb{C} \backslash \mathbb{P}_{\alpha, j}$ directly

$$
\begin{aligned}
& \left(1-\overline{b_{\alpha_{j}}(v)} b_{\alpha_{j}}(u)\right) \sum_{k=0}^{j}\left(X_{k}^{\#}(v)\right)^{*} X_{k}^{\#}(u) \\
& \quad=\left(\left(Y_{j}^{\#}\right)^{[\alpha, j]}(v)\right)^{*}\left(Y_{j}^{\#}\right)^{[\alpha, j]}(u)-\overline{b_{\alpha_{j}}(v)} b_{\alpha_{j}}(u)\left(X_{j}^{\#}(v)\right)^{*} X_{j}^{\#}(u), \\
& \left(1-b_{\alpha_{j}}(u) \overline{b_{\alpha_{j}}(v)}\right) \sum_{k=0}^{j} Y_{k}^{\#}(u)\left(Y_{k}^{\#}(v)\right)^{*} \\
& \quad=\left(X_{j}^{\#}\right)^{[\alpha, j]}(u)\left(\left(X_{j}^{\#}\right)^{[\alpha, j]}(v)\right)^{*}-b_{\alpha_{j}}(u) \overline{b_{\alpha_{j}}(v)} Y_{j}^{\#}(u)\left(Y_{j}^{\#}(v)\right)^{*} .
\end{aligned}
$$

The essential new information concerns the third and fourth Christoffel-Darboux formulae in Theorem 4.2 which can be regarded as a matricial version of [6, the first identity in Corollary 4.3.4]. Furthermore, the Christoffel-Darboux formulae in Theorem 4.2 can be obviously restated as follows.

Corollary 4.3. For each integer $\ell \in\{1,2, \ldots, \tau\}$ and points $u, v \in \mathbb{C} \backslash \mathbb{P}_{\alpha, \ell}$, the following Christoffel-Darboux formulae hold:

$$
\begin{aligned}
& \left(1-\overline{b_{\alpha_{\ell}}(v)} b_{\alpha_{\ell}}(u)\right) \sum_{k=0}^{\ell-1}\left(X_{k}(v)\right)^{*} X_{k}(u)=\left(Y_{\ell}^{[\alpha, \ell]}(v)\right)^{*} Y_{\ell}^{[\alpha, \ell]}(u)-\left(X_{\ell}(v)\right)^{*} X_{\ell}(u), \\
& \left(1-b_{\alpha_{\ell}}(u) \overline{b_{\alpha_{\ell}}(v)}\right) \sum_{k=0}^{\ell-1} Y_{k}(u)\left(Y_{k}(v)\right)^{*}=X_{\ell}^{[\alpha, \ell]}(u)\left(X_{\ell}^{[\alpha, \ell]}(v)\right)^{*}-Y_{\ell}(u)\left(Y_{\ell}(v)\right)^{*}, \\
& \left(1-\overline{b_{\alpha_{\ell}}(v)} b_{\alpha_{\ell}}(u)\right) \sum_{k=0}^{\ell-1}\left(X_{k}^{\#}(v)\right)^{*} X_{k}(u) \\
& =\frac{2\left(1-\left|\alpha_{\ell}\right|^{2}\right)}{\left(1-\alpha_{\ell} \bar{v}\right)\left(1-u \overline{\alpha_{\ell}}\right)} \mathbf{I}_{q}-\left(\left(Y_{\ell}^{\#}\right)^{[\alpha, \ell]}(v)\right)^{*} Y_{\ell}^{[\alpha, \ell]}(u)-\left(X_{\ell}^{\#}(v)\right)^{*} X_{\ell}(u), \\
& \left(1-b_{\alpha_{\ell}}(u) \overline{b_{\alpha_{\ell}}(v)}\right) \sum_{k=0}^{\ell-1} Y_{k}(u)\left(Y_{k}^{\#}(v)\right)^{*} \\
& =\frac{2\left(1-\left|\alpha_{\ell}\right|^{2}\right)}{\left(1-u \overline{\alpha_{\ell}}\right)\left(1-\alpha_{\ell} \bar{v}\right)} \mathbf{I}_{q}-X_{\ell}^{[\alpha, \ell]}(u)\left(\left(X_{\ell}^{\#}\right)^{[\alpha, \ell]}(v)\right)^{*}-Y_{\ell}(u)\left(Y_{\ell}^{\#}(v)\right)^{*} .
\end{aligned}
$$

Remark 4.4. Similarly as performed in [25, Lemma 6.5], the first (or second) Christoffel-Darboux formulae with $u=v=z, z \in \mathbb{T}$ in Corollary 4.3 yield for each integer $\ell \in$ $\{1,2, \ldots, \tau\}$ and point $u \in \mathbb{C} \backslash \mathbb{P}_{\alpha, \ell}$ an alternative approach (cf. Corollary 3.7) to the equality $X_{\ell}^{[\alpha, \ell]}(u) X_{\ell}(u)=Y_{\ell}(u) Y_{\ell}^{[\alpha, \ell]}(u)$. 
Remark 4.5. Using the same strategy as in the case of orthogonal rational matrix-valued functions (cf. [25, Section 7]), from the first (or second) Christoffel-Darboux formulae with $u=v$ in Corollary 4.3 one can conclude that, for each integer $\ell \in\{1,2, \ldots, \tau\}$ and point $u \in \mathbb{D}$, the relations $\operatorname{det} X_{\ell}^{[\alpha, \ell]}(u) \neq 0$ and $\operatorname{det} Y_{\ell}^{[\alpha, \ell]}(u) \neq 0$ are satisfied at which the complex $q \times q$ matrices $\left(X_{\ell}^{[\alpha, \ell]}(u)\right)^{-1} Y_{\ell}(u)$ and $X_{\ell}(u)\left(Y_{\ell}^{[\alpha, \ell]}(u)\right)^{-1}$ are strictly contractive (cf. Corollary 3.2).

\section{A characterization of Szegö pairs}

In the previous section (see, e.g., Theorem 4.2), we have explained that a Szegö pair of rational matrix-valued functions along with its dual Szegö pair fulfills some ChristoffelDarboux formulae. Referring to this, we study now an inverse problem. Roughly speaking, we will see that the realization of Christoffel-Darboux formulae is in a way also a sufficient condition for rational matrix-valued functions to be dual Szegö pairs of rational matrix-valued functions (cf. [27, Theorem 3.10]).

Remark 5.1. If $\ell$ is a positive integer and if $X_{k}, Y_{k}, X_{k}^{\#}, Y_{k}^{\#} \in \breve{\mathscr{R}}_{\alpha, k}^{q \times q}$ for each $k \in\{0,1, \ldots, \ell\}$ then, by setting $j:=\ell$, the following statements are equivalent.

(i) The first (resp., second, third, or fourth) formula of Theorem 4.2 is satisfied.

(ii) The first (resp., second, third, or fourth) formula of Corollary 4.3 is satisfied.

Lemma 5.2. Let $\ell$ be a positive integer and let $X_{k}, Y_{k}, X_{k}^{\#}, Y_{k}^{\#} \in \breve{\mathscr{R}}_{\alpha, k}^{q \times q}$ for $k \in\{\ell-1, \ell\}$. The following statements are equivalent.

(i) The first (resp., third) identity of Lemma 4.1 is satisfied.

(ii) The second (resp., fourth) identity of Lemma 4.1 is satisfied.

Proof. If we fix $v \in \mathbb{C} \backslash \mathbb{P}_{\alpha, \ell}$ then, in view of (2.1) and forming the adjoint with respect to the $\ell+2$ points $\alpha_{0}, \alpha_{1}, \ldots, \alpha_{\ell}, \alpha_{\ell-1}$, the first identity of Lemma 4.1 is equal to

$$
\begin{aligned}
& \left(b_{\alpha_{\ell-1}}(u)-b_{\alpha_{\ell-1}}(v)\right)\left(Y_{\ell}(u) Y_{\ell}^{[\alpha, \ell]}(v)-X_{\ell}^{[\alpha, \ell]}(u) X_{\ell}(v)\right) \\
& \quad=\left(b_{\alpha_{\ell}}(u)-b_{\alpha_{\ell}}(v)\right)\left(b_{\alpha_{\ell-1}}(u) Y_{\ell-1}(u) Y_{\ell-1}^{[\alpha, \ell-1]}(v)-b_{\alpha_{\ell-1}}(v) X_{\ell-1}^{[\alpha, \ell-1]}(u) X_{\ell-1}(v)\right) .
\end{aligned}
$$

Since, by fixing now the point $u \in \mathbb{C} \backslash \mathbb{P}_{\alpha, \ell}$ and adjoining, this relation is equal to

$$
\begin{aligned}
& \left(b_{\alpha_{\ell-1}}(v) \overline{b_{\alpha_{\ell-1}}(u)}-1\right)\left(Y_{\ell}(v)\left(Y_{\ell}(u)\right)^{*}-X_{\ell}^{[\alpha, \ell]}(v)\left(X_{\ell}^{[\alpha, \ell]}(u)\right)^{*}\right) \\
& \quad=\left(b_{\alpha_{\ell}}(v) \overline{b_{\alpha_{\ell}}(u)}-1\right)\left(\overline{b_{\alpha_{\ell-1}}(u)} b_{\alpha_{\ell-1}}(v) Y_{\ell-1}(v)\left(Y_{\ell-1}(u)\right)^{*}-X_{\ell-1}^{[\alpha, \ell-1]}(v)\left(X_{\ell-1}^{[\alpha, \ell-1]}(u)\right)^{*}\right),
\end{aligned}
$$

we obtain the equivalence of the first and the second identity of Lemma 4.1. Similarly, one can conclude that the third and the fourth identity of Lemma 4.1 are equivalent.

Lemma 5.3. Let $X_{\ell}, Y_{\ell}, X_{\ell}^{\#}, Y_{\ell}^{\#} \in \breve{\mathscr{R}}_{\alpha, \ell}^{q \times q}$ for each integer $\ell \in\{1,2, \ldots, \tau\}$ and let $X_{0}, Y_{0}, X_{0}^{\#}, Y_{0}^{\#}$ be the rational matrix-valued functions defined as in (2.4) and (2.6) for some nonsingular complex $q \times q$ matrices $\mathbf{X}_{0}, \mathbf{Y}_{0}$ satisfying the condition $\mathbf{X}_{0}^{*} \mathbf{X}_{0}=\mathbf{Y}_{0} \mathbf{Y}_{0}^{*}$. The following 
statements are equivalent.

(i) For each integer $\ell \in\{1,2, \ldots, \tau\}$, the first (resp., second, third, or fourth) identity of Lemma 4.1 is fulfilled.

(ii) For each integer $j \in\{1,2, \ldots, \tau\}$, the first (resp., second, third, or fourth) identity of Theorem 4.2 is fulfilled.

Proof. Using the same arguments as in the proof of Theorem 4.2, one can inductively show that (i) implies (ii). It remains to verify that (ii) implicates also (i). Note that the choice of $X_{0}, Y_{0}$ supplies immediately that the identities of Theorem 4.2 are also satisfied for $j=0$ (cf. the proof of Theorem 4.2). Thus, for each integer $\ell \in\{1,2, \ldots, \tau\}$ and points $u, v \in \mathbb{C} \backslash \mathbb{P}_{\alpha, \ell}$, from (4.1), (ii), Remark 5.1, the fourth identity of Corollary 4.3, and the fourth identity of Theorem 4.2 , it follows that

$$
\begin{aligned}
k_{\alpha_{\ell-1}}(u, v)\left(X_{\ell}^{[\alpha, \ell]}(u)\left(\left(X_{\ell}^{\#}\right)^{[\alpha, \ell]}(v)\right)^{*}+Y_{\ell}(u)\left(Y_{\ell}^{\#}(v)\right)^{*}\right) \\
=-k_{\alpha_{\ell-1}}(u, v)\left(\frac{2\left(1-\left|\alpha_{\ell}\right|^{2}\right)}{\left(1-u \overline{\alpha_{\ell}}\right)\left(1-\alpha_{\ell} \bar{v}\right)} \mathbf{I}_{q}-X_{\ell}^{[\alpha, \ell]}(u)\left(\left(X_{\ell}^{\#}\right)^{[\alpha, \ell]}(v)\right)^{*}-Y_{\ell}(u)\left(Y_{\ell}^{\#}(v)\right)^{*}\right) \\
\quad+\frac{2\left(1-\left|\alpha_{\ell}\right|^{2}\right)\left(1-\left|\alpha_{\ell-1}\right|^{2}\right)(1-u \bar{v})}{\left(1-u \overline{\alpha_{\ell}}\right)\left(1-\alpha_{\ell} \bar{v}\right)\left(1-u \overline{\alpha_{\ell-1}}\right)\left(1-\alpha_{\ell-1} \bar{v}\right)} \mathbf{I}_{q} \\
=k_{\alpha_{\ell-1}}(u, v) k_{\alpha_{\ell}}(u, v) \sum_{k=0}^{\ell-1} Y_{k}(u)\left(Y_{k}^{\#}(v)\right)^{*}+k_{\alpha_{\ell}}(u, v) \frac{2\left(1-\left|\alpha_{\ell-1}\right|^{2}\right)}{\left(1-u \overline{\alpha_{\ell-1}}\right)\left(1-\alpha_{\ell-1} \bar{v}\right)} \mathbf{I}_{q} \\
=k_{\alpha_{\ell}}(u, v)\left(X_{\ell-1}^{[\alpha, \ell-1]}(u)\left(\left(X_{\ell-1}^{\#}\right)^{[\alpha, \ell-1]}(v)\right)^{*}+b_{\alpha_{\ell-1}}(u) \overline{b_{\alpha_{\ell-1}}(v)} Y_{\ell-1}(u)\left(Y_{\ell-1}^{\#}(v)\right)^{*}\right) .
\end{aligned}
$$

Consequently, with respect to the fourth kind of identities it is shown that (ii) yields (i). Similarly by a straightforward calculation, one can prove this implication referring to the first, second, and third kind of identities, respectively.

Theorem 5.4. Let $\left(X_{j}\right)_{j=0}^{\tau},\left(Y_{j}\right)_{j=0}^{\tau}$ be such that $X_{j}, Y_{j} \in \breve{\mathscr{R}}_{\alpha, j}^{q \times q}$ and the first (or second) identity of Theorem 4.2 is fulfilled for each integer $j \in\{0,1, \ldots, \tau\}$ as well as that $X_{0}\left(\alpha_{0}\right)$, $Y_{0}\left(\alpha_{0}\right)$ are nonsingular matrices. Then the matrices $X_{\ell}^{[\alpha, \ell]}\left(\alpha_{\ell-1}\right), Y_{\ell}^{[\alpha, \ell]}\left(\alpha_{\ell-1}\right)$ are nonsingular for each integer $\ell \in\{1,2, \ldots, \tau\}$ and if in addition

$$
\frac{\eta_{\ell} \overline{\eta_{\ell-1}}}{1-\overline{\alpha_{\ell}} \alpha_{\ell-1}}\left(X_{\ell}^{[\alpha, \ell]}\left(\alpha_{\ell-1}\right)\right)^{-1} X_{\ell-1}^{[\alpha, \ell-1]}\left(\alpha_{\ell-1}\right), \quad \frac{\eta_{\ell} \overline{\eta_{\ell-1}}}{1-\overline{\alpha_{\ell}} \alpha_{\ell-1}} Y_{\ell-1}^{[\alpha, \ell-1]}\left(\alpha_{\ell-1}\right)\left(Y_{\ell}^{[\alpha, \ell]}\left(\alpha_{\ell-1}\right)\right)^{-1}
$$

are positive Hermitian matrices and if in that case

$$
\mathbf{E}_{\ell}:=\eta_{\ell} \overline{\eta_{\ell-1}}\left(X_{\ell}^{[\alpha, \ell]}\left(\alpha_{\ell-1}\right)\right)^{-1} Y_{\ell}\left(\alpha_{\ell-1}\right), \quad \ell \in\{1,2, \ldots, \tau\}
$$

$\mathbf{X}_{0}:=\sqrt{1-\left|\alpha_{0}\right|^{2}} X_{0}\left(\alpha_{0}\right)$, and $\mathbf{Y}_{0}:=\sqrt{1-\left|\alpha_{0}\right|^{2}} Y_{0}\left(\alpha_{0}\right)$, then $\left[\left(X_{j}\right)_{j=0}^{\tau},\left(Y_{j}\right)_{j=0}^{\tau}\right]$ is the Szegö pair of rational matrix-valued functions generated by $\left[\left(\alpha_{j}\right)_{j=0}^{\tau} ;\left(\mathbf{E}_{\ell}\right)_{\ell=1}^{\tau} ; \mathbf{X}_{0}, \mathbf{Y}_{0}\right]$. Moreover in that 
case, if $\left(X_{j}^{\#}\right)_{j=0}^{\tau},\left(Y_{j}^{\#}\right)_{j=0}^{\tau}$ are chosen such that $X_{j}^{\#}, Y_{j}^{\#} \in \breve{\mathscr{R}}_{\alpha, j}^{q \times q}$ and (4.7) (or (4.8)) is fulfilled for each integer $j \in\{0,1, \ldots, \tau\}$, then the following statements are equivalent.

(i) $\left[\left(X_{j}^{\#}\right)_{j=0}^{\tau},\left(Y_{j}^{\#}\right)_{j=0}^{\tau}\right]$ is the dual Szegö pair of $\left[\left(X_{j}\right)_{j=0}^{\tau},\left(Y_{j}\right)_{j=0}^{\tau}\right]$.

(ii) $\left(X_{0}^{\#}\left(\alpha_{0}\right)\right)^{*} X_{0}^{\#}\left(\alpha_{0}\right)=\left(1-\left|\alpha_{0}\right|^{2}\right)\left(X_{0}^{\#}\left(\alpha_{0}\right)\right)^{*} X_{0}^{\#}\left(\alpha_{0}\right) \mathbf{X}_{0}^{*} \mathbf{X}_{0}\left(X_{0}^{\#}\left(\alpha_{0}\right)\right)^{*} X_{0}^{\#}\left(\alpha_{0}\right)$ (or $Y_{0}^{\#}\left(\alpha_{0}\right)$ $\left.\left(Y_{0}^{\#}\left(\alpha_{0}\right)\right)^{*}=\left(1-\left|\alpha_{0}\right|^{2}\right) Y_{0}^{\#}\left(\alpha_{0}\right)\left(Y_{0}^{\#}\left(\alpha_{0}\right)\right)^{*} \mathbf{Y}_{0} \mathbf{Y}_{0}^{*} Y_{0}^{\#}\left(\alpha_{0}\right)\left(Y_{0}^{\#}\left(\alpha_{0}\right)\right)^{*}\right)$ and the third (or fourth) identity of Theorem 4.2 is fulfilled for each integer $j \in\{0,1, \ldots, \tau\}$.

(iii) $X_{0}^{\#}\left(\alpha_{0}\right)=1 /\left(1-\left|\alpha_{0}\right|^{2}\right)\left(\left(X_{0}\left(\alpha_{0}\right)\right)^{-1}\right)^{*}, Y_{0}^{\#}\left(\alpha_{0}\right)=1 /\left(1-\left|\alpha_{0}\right|^{2}\right)\left(\left(Y_{0}\left(\alpha_{0}\right)\right)^{-1}\right)^{*}$, and for each integer $\ell \in\{1,2, \ldots, \tau\}$ the three relations below are satisfied:

$$
\begin{gathered}
\left(X_{\ell}^{[\alpha, \ell]}\left(\alpha_{\ell-1}\right)\right)^{-1} Y_{\ell}\left(\alpha_{\ell-1}\right)=-\left(\left(X_{\ell}^{\#}\right)^{[\alpha, \ell]}\left(\alpha_{\ell-1}\right)\right)^{-1} Y_{\ell}^{\#}\left(\alpha_{\ell-1}\right), \\
\left(X_{\ell}^{[\alpha, \ell]}\left(\alpha_{\ell-1}\right)\right)^{-1} X_{\ell-1}^{[\alpha, \ell-1]}\left(\alpha_{\ell-1}\right)=\left(\left(X_{\ell}^{\#}\right)^{[\alpha, \ell]}\left(\alpha_{\ell-1}\right)\right)^{-1}\left(X_{\ell-1}^{\#}\right)^{[\alpha, \ell-1]}\left(\alpha_{\ell-1}\right), \\
Y_{\ell-1}^{[\alpha, \ell-1]}\left(\alpha_{\ell-1}\right)\left(Y_{\ell}^{[\alpha, \ell]}\left(\alpha_{\ell-1}\right)\right)^{-1}=\left(Y_{\ell-1}^{\#}\right)^{[\alpha, \ell-1]}\left(\alpha_{\ell-1}\right)\left(\left(Y_{\ell}^{\#}\right)^{[\alpha, \ell]}\left(\alpha_{\ell-1}\right)\right)^{-1} .
\end{gathered}
$$

Proof. First, we consider the case $j=0$. Since $X_{0}, Y_{0} \in \breve{\mathscr{T}}_{\alpha, 0}^{q \times q}$, the definitions

$$
\mathbf{X}_{0}:=\sqrt{1-\left|\alpha_{0}\right|^{2}} X_{0}\left(\alpha_{0}\right), \quad \mathbf{Y}_{0}:=\sqrt{1-\left|\alpha_{0}\right|^{2}} Y_{0}\left(\alpha_{0}\right)
$$

imply immediately that $X_{0}$ and $Y_{0}$ admit the representations in (2.4), where $\mathbf{X}_{0}$ and $\mathbf{Y}_{0}$ are nonsingular complex $q \times q$ matrices. Furthermore, from the first (resp., second) identity of Theorem 4.2 we get

$$
\begin{gathered}
\left(X_{0}(v)\right)^{*} X_{0}(u)=\left(Y_{0}^{[\alpha, 0]}(v)\right)^{*} Y_{0}^{[\alpha, 0]}(u) \\
\left(\text { resp., } Y_{0}(u)\left(Y_{0}(v)\right)^{*}=X_{0}^{[\alpha, 0]}(u)\left(X_{0}^{[\alpha, 0]}(v)\right)^{*}\right)
\end{gathered}
$$

for each $u, v \in \mathbb{C} \backslash \mathbb{P}_{\alpha, 0}$. Hence, in view of (2.1) and (2.4), it follows $\mathbf{X}_{0}^{*} \mathbf{X}_{0}=\mathbf{Y}_{0} \mathbf{Y}_{0}^{*}$. Particularly for the case $\tau=0$ it is shown that $\left[\left(X_{j}\right)_{j=0}^{\tau},\left(Y_{j}\right)_{j=0}^{\tau}\right]$ is the Szegö pair of rational matrix-valued functions generated by $\left[\left(\alpha_{j}\right)_{j=0}^{\tau} ;\left(\mathbf{E}_{\ell}\right)_{\ell=1}^{\tau} ; \mathbf{X}_{0}, \mathbf{Y}_{0}\right]$.

Now let $\tau$ be a positive integer or $\infty$ and let $\ell$ be an integer belonging to $\{1,2, \ldots, \tau\}$. Because of the choice of $\left(X_{j}\right)_{j=0}^{\tau}$ and $\left(Y_{j}\right)_{j=0}^{\tau}$, Lemmas 5.2 and 5.3 (which include especially that at any rate the first identity of Lemma 4.1 is fulfilled), Remarks 5.1 and 4.5 we obtain that the matrices $X_{\ell}^{[\alpha, \ell]}(u), Y_{\ell}^{[\alpha, \ell]}(u)$ are nonsingular for each $u \in \mathbb{D}$ and by using $b_{\alpha_{\ell-1}}\left(\alpha_{\ell-1}\right)=0$ that

$$
\begin{aligned}
& \left(Y_{\ell}^{[\alpha, \ell]}\left(\alpha_{\ell-1}\right)\right)^{*} Y_{\ell}^{[\alpha, \ell]}(u)-\left(X_{\ell}\left(\alpha_{\ell-1}\right)\right)^{*} X_{\ell}(u) \\
& \quad=\left(1-b_{\alpha_{\ell-1}}(u) \overline{b_{\alpha_{\ell-1}}\left(\alpha_{\ell-1}\right)}\right)\left(\left(Y_{\ell}^{[\alpha, \ell]}\left(\alpha_{\ell-1}\right)\right)^{*} Y_{\ell}^{[\alpha, \ell]}(u)-\left(X_{\ell}\left(\alpha_{\ell-1}\right)\right)^{*} X_{\ell}(u)\right) \\
& \quad=\left(1-b_{\alpha_{\ell}}(u) \overline{b_{\alpha_{\ell}}\left(\alpha_{\ell-1}\right)}\right)\left(Y_{\ell-1}^{[\alpha, \ell-1]}\left(\alpha_{\ell-1}\right)\right)^{*} Y_{\ell-1}^{[\alpha, \ell-1]}(u)
\end{aligned}
$$


for all $u \in \mathbb{C} \backslash \mathbb{P}_{\alpha, \ell}$. Notably, we have that $X_{\ell}^{[\alpha, \ell]}\left(\alpha_{\ell-1}\right), Y_{\ell}^{[\alpha, \ell]}\left(\alpha_{\ell-1}\right)$ are nonsingular as well as by the choice of $\mathbf{E}_{\ell}$, Remarks 4.4, 4.5, and 5.1 that

$$
\mathbf{E}_{\ell}=\eta_{\ell} \overline{\eta_{\ell-1}}\left(X_{\ell}^{[\alpha, \ell]}\left(\alpha_{\ell-1}\right)\right)^{-1} Y_{\ell}\left(\alpha_{\ell-1}\right)=\eta_{\ell} \overline{\eta_{\ell-1}} X_{\ell}\left(\alpha_{\ell-1}\right)\left(Y_{\ell}^{[\alpha, \ell]}\left(\alpha_{\ell-1}\right)\right)^{-1}
$$

and therefore

$$
\begin{aligned}
& \left(Y_{\ell}^{[\alpha, \ell]}\left(\alpha_{\ell-1}\right)\right)^{*}\left(\mathbf{I}_{q}-\mathbf{E}_{\ell}^{*} \mathbf{E}_{\ell}\right) Y_{\ell}^{[\alpha, \ell]}\left(\alpha_{\ell-1}\right) \\
& \quad=\left(Y_{\ell}^{[\alpha, \ell]}\left(\alpha_{\ell-1}\right)\right)^{*} Y_{\ell}^{[\alpha, \ell]}\left(\alpha_{\ell-1}\right)-\left(X_{\ell}\left(\alpha_{\ell-1}\right)\right)^{*} X_{\ell}\left(\alpha_{\ell-1}\right) \\
& \quad=\left(1-\left|b_{\alpha_{\ell}}\left(\alpha_{\ell-1}\right)\right|^{2}\right)\left(Y_{\ell-1}^{[\alpha, \ell-1]}\left(\alpha_{\ell-1}\right)\right)^{*} Y_{\ell-1}^{[\alpha, \ell-1]}\left(\alpha_{\ell-1}\right) .
\end{aligned}
$$

This implies that $\mathbf{E}_{\ell}$ is a strictly contractive $q \times q$ matrix and that

$$
\left(\mathbf{I}_{q}-\mathbf{E}_{\ell}^{*} \mathbf{E}_{\ell}\right)^{1 / 2}=\frac{\eta_{\ell} \overline{\eta_{\ell-1}} \sqrt{\left(1-\left|\alpha_{\ell}\right|^{2}\right)\left(1-\left|\alpha_{\ell-1}\right|^{2}\right)}}{1-\overline{\alpha_{\ell}} \alpha_{\ell-1}} Y_{\ell-1}^{[\alpha, \ell-1]}\left(\alpha_{\ell-1}\right)\left(Y_{\ell}^{[\alpha, \ell]}\left(\alpha_{\ell-1}\right)\right)^{-1}
$$

in view of (4.1) and the assumption that

$$
\frac{\eta_{\ell} \overline{\eta_{\ell-1}}}{1-\overline{\alpha_{\ell}} \alpha_{\ell-1}} Y_{\ell-1}^{[\alpha, \ell-1]}\left(\alpha_{\ell-1}\right)\left(Y_{\ell}^{[\alpha, \ell]}\left(\alpha_{\ell-1}\right)\right)^{-1}
$$

is a positive Hermitian matrix. Moreover, (5.9) yields by virtue of (2.1) the equality

$$
Y_{\ell}(u) Y_{\ell}^{[\alpha, \ell]}\left(\alpha_{\ell-1}\right)-X_{\ell}^{[\alpha, \ell]}(u) X_{\ell}\left(\alpha_{\ell-1}\right)=\left(b_{\alpha_{\ell}}(u)-b_{\alpha_{\ell}}\left(\alpha_{\ell-1}\right)\right) Y_{\ell-1}(u) Y_{\ell-1}^{[\alpha, \ell-1]}\left(\alpha_{\ell-1}\right)
$$

for each $u \in \mathbb{C} \backslash \mathbb{P}_{\alpha, \ell}$. Consequently, an application of (5.10) and (5.12) provides

$$
\begin{aligned}
\eta_{\ell} \overline{\eta_{\ell-1}} Y_{\ell}(u)-X_{\ell}^{[\alpha, \ell]}(u) \mathbf{E}_{\ell} \\
=\eta_{\ell} \overline{\eta_{\ell-1}} Y_{\ell}(u)-\eta_{\ell} \overline{\eta_{\ell-1}} X_{\ell}^{[\alpha, \ell]}(u) X_{\ell}\left(\alpha_{\ell-1}\right)\left(Y_{\ell}^{[\alpha, \ell]}\left(\alpha_{\ell-1}\right)\right)^{-1} \\
=\eta_{\ell} \overline{\eta_{\ell-1}}\left(b_{\alpha_{\ell}}(u)-b_{\alpha_{\ell}}\left(\alpha_{\ell-1}\right)\right) Y_{\ell-1}(u) Y_{\ell-1}^{[\alpha, \ell-1]}\left(\alpha_{\ell-1}\right)\left(Y_{\ell}^{[\alpha, \ell]}\left(\alpha_{\ell-1}\right)\right)^{-1} \\
=\frac{\left(1-\overline{\alpha_{\ell}} \alpha_{\ell-1}\right)\left(b_{\alpha_{\ell}}(u)-b_{\alpha_{\ell}}\left(\alpha_{\ell-1}\right)\right)}{\sqrt{\left(1-\left|\alpha_{\ell}\right|^{2}\right)\left(1-\left|\alpha_{\ell-1}\right|^{2}\right)}} Y_{\ell-1}(u)\left(\mathbf{I}_{q}-\mathbf{E}_{\ell}^{*} \mathbf{E}_{\ell}\right)^{1 / 2}
\end{aligned}
$$

for each $u \in \mathbb{C} \backslash \mathbb{P}_{\alpha, \ell}$. Similarly, by using Lemmas 5.2, 5.3, Remarks 4.5, and 5.1, based on 
the second identity of Lemma 4.1 one can obtain that

$$
\left(\mathbf{I}_{q}-\mathbf{E}_{\ell} \mathbf{E}_{\ell}^{*}\right)^{1 / 2}=\frac{\eta_{\ell} \overline{\eta_{\ell-1}} \sqrt{\left(1-\left|\alpha_{\ell}\right|^{2}\right)\left(1-\left|\alpha_{\ell-1}\right|^{2}\right)}}{1-\overline{\alpha_{\ell}} \alpha_{\ell-1}}\left(X_{\ell}^{[\alpha, \ell]}\left(\alpha_{\ell-1}\right)\right)^{-1} X_{\ell-1}^{[\alpha, \ell-1]}\left(\alpha_{\ell-1}\right)
$$

and that the recurrence relation

$$
\eta_{\ell} \overline{\eta_{\ell-1}} X_{\ell}(u)-\mathbf{E}_{\ell} Y_{\ell}^{[\alpha, \ell]}(u)=\frac{\left(1-\overline{\alpha_{\ell}} \alpha_{\ell-1}\right)\left(b_{\alpha_{\ell}}(u)-b_{\alpha_{\ell}}\left(\alpha_{\ell-1}\right)\right)}{\sqrt{\left(1-\left|\alpha_{\ell}\right|^{2}\right)\left(1-\left|\alpha_{\ell-1}\right|^{2}\right)}}\left(\mathbf{I}_{q}-\mathbf{E}_{\ell} \mathbf{E}_{\ell}^{*}\right)^{1 / 2} X_{\ell-1}(u)
$$

is satisfied for each $u \in \mathbb{C} \backslash \mathbb{P}_{\alpha, \ell}$. In the end, from Proposition 2.4 one can conclude that $\left[\left(X_{j}\right)_{j=0}^{\tau},\left(Y_{j}\right)_{j=0}^{\tau}\right]$ is the Szegö pair of rational matrix-valued functions generated by $\left[\left(\alpha_{j}\right)_{j=0}^{\tau} ;\left(\mathbf{E}_{\ell}\right)_{\ell=1}^{\tau} ; \mathbf{X}_{0}, \mathbf{Y}_{0}\right]$.

It remains to prove the equivalence of (i), (ii), and (iii). Because of (2.6), (5.7), and Theorem 4.2 the statement (ii) follows from (i). Now we assume (ii). Since the functions $X_{0}^{\#}$ and $Y_{0}^{\#}$ belong to $\breve{\mathscr{R}}_{\alpha, 0}^{q \times q}$, the definitions

$$
\mathbf{X}_{0}^{\#}:=\sqrt{1-\left|\alpha_{0}\right|^{2}} X_{0}^{\#}\left(\alpha_{0}\right), \quad \mathbf{Y}_{0}^{\#}:=\sqrt{1-\left|\alpha_{0}\right|^{2}} Y_{0}^{\#}\left(\alpha_{0}\right)
$$

imply immediately that $X_{0}^{\#}$ and $Y_{0}^{\#}$ admit the representations

$$
X_{0}^{\#}(u):=\frac{\sqrt{1-\left|\alpha_{0}\right|^{2}}}{1-\overline{\alpha_{0}} u} \mathbf{X}_{0}^{\#}, \quad Y_{0}^{\#}(u):=\frac{\sqrt{1-\left|\alpha_{0}\right|^{2}}}{1-\overline{\alpha_{0}} u} \mathbf{Y}_{0}^{\#}, \quad u \in \mathbb{C} \backslash \mathbb{P}_{\alpha, 0},
$$

where $\mathbf{X}_{0}^{\#}$ and $\mathbf{Y}_{0}^{\#}$ are some complex $q \times q$ matrices. From (2.1), (5.19), and the identity (4.7) (or (4.8)) with $j=0$ we get, similar as above,

$$
\left(\mathbf{X}_{0}^{\#}\right)^{*} \mathbf{X}_{0}^{\#}=\mathbf{Y}_{0}^{\#}\left(\mathbf{Y}_{0}^{\#}\right)^{*}
$$

as well as, in view of the third (or fourth) identity of Theorem 4.2, it follows that

$$
\left(\mathbf{X}_{0}^{\#}\right)^{*} \mathbf{X}_{0}+\mathbf{Y}_{0}^{\#} \mathbf{Y}_{0}^{*}=2 \mathbf{I}_{q} \quad\left(\text { or } \mathbf{X}_{0}^{*} \mathbf{X}_{0}^{\#}+\mathbf{Y}_{0}\left(\mathbf{Y}_{0}^{\#}\right)^{*}=2 \mathbf{I}_{q}\right)
$$

Thus, we have at any rate

$$
\left(\begin{array}{cc}
-\mathbf{Y}_{0} & \mathbf{X}_{0}^{*} \\
\mathbf{Y}_{0}^{\#} & \left(\mathbf{X}_{0}^{\#}\right)^{*}
\end{array}\right)\left(\begin{array}{cc}
-\left(\mathbf{Y}_{0}^{\#}\right)^{*} & \mathbf{Y}_{0}^{*} \\
\mathbf{X}_{0}^{\#} & \mathbf{X}_{0}
\end{array}\right)=2\left(\begin{array}{cc}
\mathbf{I}_{q} & \mathbf{0}_{q} \\
\mathbf{0}_{q} & \mathbf{I}_{q}
\end{array}\right)
$$

which implicates

$$
\left(\begin{array}{cc}
-\left(\mathbf{Y}_{0}^{\#}\right)^{*} & \mathbf{Y}_{0}^{*} \\
\mathbf{X}_{0}^{\#} & \mathbf{X}_{0}
\end{array}\right)\left(\begin{array}{cc}
-\mathbf{Y}_{0} & \mathbf{X}_{0}^{*} \\
\mathbf{Y}_{0}^{\#} & \left(\mathbf{X}_{0}^{\#}\right)^{*}
\end{array}\right)=2\left(\begin{array}{cc}
\mathbf{I}_{q} & \mathbf{0}_{q} \\
\mathbf{0}_{q} & \mathbf{I}_{q}
\end{array}\right)
$$


In particular, the equality

$$
\mathbf{X}_{0}^{\#} \mathbf{X}_{0}^{*}+\mathbf{X}_{0}\left(\mathbf{X}_{0}^{\#}\right)^{*}=2 \mathbf{I}_{q}
$$

is fulfilled. Moreover, by virtue of (5.18) and (5.20) we see that the relation

$$
\left(X_{0}^{\#}\left(\alpha_{0}\right)\right)^{*} X_{0}^{\#}\left(\alpha_{0}\right)=\left(1-\left|\alpha_{0}\right|^{2}\right)\left(X_{0}^{\#}\left(\alpha_{0}\right)\right)^{*} X_{0}^{\#}\left(\alpha_{0}\right) \mathbf{X}_{0}^{*} \mathbf{X}_{0}\left(X_{0}^{\#}\left(\alpha_{0}\right)\right)^{*} X_{0}^{\#}\left(\alpha_{0}\right)
$$

is tantamount to

$$
Y_{0}^{\#}\left(\alpha_{0}\right)\left(Y_{0}^{\#}\left(\alpha_{0}\right)\right)^{*}=\left(1-\left|\alpha_{0}\right|^{2}\right) Y_{0}^{\#}\left(\alpha_{0}\right)\left(Y_{0}^{\#}\left(\alpha_{0}\right)\right)^{*} \mathbf{Y}_{0} \mathbf{Y}_{0}^{*} Y_{0}^{\#}\left(\alpha_{0}\right)\left(Y_{0}^{\#}\left(\alpha_{0}\right)\right)^{*}
$$

Because (5.24) supplies that the real part of $\mathbf{X}_{0}^{\#} \mathbf{X}_{0}^{*}$ is a positive Hermitian matrix, [11, Lemma 1.1.13 (c)] shows that even $\mathbf{X}_{0}^{\#}$ is a nonsingular matrix. Hence, (5.18) and (5.25) yield $\left(\mathbf{X}_{0}^{\#}\right)^{*} \mathbf{X}_{0}^{\#}=\left(\mathbf{X}_{0}^{*} \mathbf{X}_{0}\right)^{-1}$ such that from (5.24) one can get

$$
\left(\mathbf{I}_{q}-\mathbf{X}_{0}\left(\mathbf{X}_{0}^{\#}\right)^{*}\right)^{2}=\mathbf{0}_{q}
$$

Using this equality in combination with the fact that the real part of $\mathbf{I}_{q}-\mathbf{X}_{0}\left(\mathbf{X}_{0}^{\#}\right) *$ is $\mathbf{0}_{q}$ (cf. (5.24)) one can conclude $\mathbf{X}_{0}^{\#}=\left(\mathbf{X}_{0}^{-1}\right)^{*}$. Therefore, from (5.21) it follows in addition that $\mathbf{Y}_{0}^{\#}=\left(\mathbf{Y}_{0}^{-1}\right)^{*}$. In view of (5.7) and (5.18), we have

$$
X_{0}^{\#}\left(\alpha_{0}\right)=\frac{1}{1-\left|\alpha_{0}\right|^{2}}\left(\left(X_{0}\left(\alpha_{0}\right)\right)^{-1}\right)^{*}, \quad Y_{0}^{\#}\left(\alpha_{0}\right)=\frac{1}{1-\left|\alpha_{0}\right|^{2}}\left(\left(Y_{0}\left(\alpha_{0}\right)\right)^{-1}\right)^{*} \text {. }
$$

Since the identity (4.7) (or (4.8)) is fulfilled for each integer $j \in\{0,1, \ldots, \tau\}$, as above we see at least from Remark 4.5 that $\left(X_{\ell}^{\#}\right)^{[\alpha, \ell]}\left(\alpha_{\ell-1}\right),\left(Y_{\ell}^{\#}\right)^{[\alpha, \ell]}\left(\alpha_{\ell-1}\right)$ are nonsingular matrices for each integer $\ell \in\{1,2, \ldots, \tau\}$. Because of (ii), Lemmas 5.2, 5.3 (which include that at any rate the third identity of Lemma 4.1 is fulfilled), and $b_{\alpha_{\ell-1}}\left(\alpha_{\ell-1}\right)=0$ one can reason

$$
\begin{aligned}
& \left(\left(Y_{\ell}^{\#}\right)^{[\alpha, \ell]}\left(\alpha_{\ell-1}\right)\right)^{*} Y_{\ell}^{[\alpha, \ell]}(u)+\left(X_{\ell}^{\#}\left(\alpha_{\ell-1}\right)\right)^{*} X_{\ell}(u) \\
& \quad=\left(1-b_{\alpha_{\ell}}(u) \overline{b_{\alpha_{\ell}}\left(\alpha_{\ell-1}\right)}\right)\left(\left(Y_{\ell-1}^{\#}\right)^{[\alpha, \ell-1]}\left(\alpha_{\ell-1}\right)\right)^{*} Y_{\ell-1}^{[\alpha, \ell-1]}(u)
\end{aligned}
$$

for each integer $\ell \in\{1,2, \ldots, \tau\}$ and point $u \in \mathbb{C} \backslash \mathbb{P}_{\alpha, \ell}$. By virtue of $(2.1)$ we get

$$
\begin{aligned}
Y_{\ell}(u) & \left(Y_{\ell}^{\#}\right)^{[\alpha, \ell]}\left(\alpha_{\ell-1}\right)+X_{\ell}^{[\alpha, \ell]}(u) X_{\ell}^{\#}\left(\alpha_{\ell-1}\right) \\
& =\left(b_{\alpha_{\ell}}(u)-b_{\alpha_{\ell}}\left(\alpha_{\ell-1}\right)\right) Y_{\ell-1}(u)\left(Y_{\ell-1}^{\#}\right)^{[\alpha, \ell-1]}\left(\alpha_{\ell-1}\right)
\end{aligned}
$$


for each integer $\ell \in\{1,2, \ldots, \tau\}$ and point $u \in \mathbb{C} \backslash \mathbb{P}_{\alpha, \ell}$. Choosing in this equality at first $u=\alpha_{\ell-1}$, one can see

$$
\left(X_{\ell}^{[\alpha, \ell]}\left(\alpha_{\ell-1}\right)\right)^{-1} Y_{\ell}\left(\alpha_{\ell-1}\right)=-X_{\ell}^{\#}\left(\alpha_{\ell-1}\right)\left(\left(Y_{\ell}^{\#}\right)^{[\alpha, \ell]}\left(\alpha_{\ell-1}\right)\right)^{-1}
$$

and then in view of (5.10), (5.12), and (5.15) also

$$
Y_{\ell-1}^{[\alpha, \ell-1]}\left(\alpha_{\ell-1}\right)\left(Y_{\ell}^{[\alpha, \ell]}\left(\alpha_{\ell-1}\right)\right)^{-1}=\left(Y_{\ell-1}^{\#}\right)^{[\alpha, \ell-1]}\left(\alpha_{\ell-1}\right)\left(\left(Y_{\ell}^{\#}\right)^{[\alpha, \ell]}\left(\alpha_{\ell-1}\right)\right)^{-1}
$$

for each integer $\ell \in\{1,2, \ldots, \tau\}$. Similarly, by using Lemmas 5.2, 5.3, and based on the fourth identity of Lemma 4.1 one can obtain that

$$
\begin{gathered}
X_{\ell}\left(\alpha_{\ell-1}\right)\left(Y_{\ell}^{[\alpha, \ell]}\left(\alpha_{\ell-1}\right)\right)^{-1}=-\left(\left(X_{\ell}^{\#}\right)^{[\alpha, \ell]}\left(\alpha_{\ell-1}\right)\right)^{-1} Y_{\ell}^{\#}\left(\alpha_{\ell-1}\right), \\
\left(X_{\ell}^{[\alpha, \ell]}\left(\alpha_{\ell-1}\right)\right)^{-1} X_{\ell-1}^{[\alpha, \ell-1]}\left(\alpha_{\ell-1}\right)=\left(\left(X_{\ell}^{\#}\right)^{[\alpha, \ell]}\left(\alpha_{\ell-1}\right)\right)^{-1}\left(X_{\ell-1}^{\#}\right)^{[\alpha, \ell-1]}\left(\alpha_{\ell-1}\right) .
\end{gathered}
$$

Consequently (note (5.28) and again (5.10)), the statement (iii) is fulfilled. Finally, we show that (iii) implies (i). Since $X_{0}^{\#}, Y_{0}^{\#} \in \breve{\mathscr{R}}_{\alpha, 0}^{q \times q}$, the relations (5.7) and (5.28) imply that $X_{0}^{\#}$ and $Y_{0}^{\#}$ admit the representations in (5.7). Furthermore, from (iii), the choice of $\mathbf{E}_{\ell}$, (5.12), and (5.16), it follows that

$$
\begin{aligned}
& -\mathbf{E}_{\ell}=-\eta_{\ell} \overline{\eta_{\ell-1}}\left(X_{\ell}^{[\alpha, \ell]}\left(\alpha_{\ell-1}\right)\right)^{-1} Y_{\ell}\left(\alpha_{\ell-1}\right)=\eta_{\ell} \overline{\eta_{\ell-1}}\left(\left(X_{\ell}^{\#}\right)^{[\alpha, \ell]}\left(\alpha_{\ell-1}\right)\right)^{-1} Y_{\ell}^{\#}\left(\alpha_{\ell-1}\right), \\
& \left(\mathbf{I}_{q}-\mathbf{E}_{\ell} \mathbf{E}_{\ell}^{*}\right)^{1 / 2} \\
& \left.\quad=\frac{\eta_{\ell} \overline{\eta_{\ell-1}} \sqrt{\left(1-\left|\alpha_{\ell}\right|^{2}\right)\left(1-\left|\alpha_{\ell-1}\right|^{2}\right)}}{1-\overline{\alpha_{\ell}} \alpha_{\ell-1}}\left(X_{\ell}^{\#}\right)^{[\alpha, \ell]}\left(\alpha_{\ell-1}\right)\right)^{-1}\left(X_{\ell-1}^{\#}\right)^{[\alpha, \ell-1]}\left(\alpha_{\ell-1}\right), \\
& \left(\mathbf{I}_{q}-\mathbf{E}_{\ell}^{*} \mathbf{E}_{\ell}\right)^{1 / 2} \\
& \quad=\frac{\eta_{\ell} \overline{\eta_{\ell-1}} \sqrt{\left(1-\left|\alpha_{\ell}\right|^{2}\right)\left(1-\left|\alpha_{\ell-1}\right|^{2}\right)}}{1-\overline{\alpha_{\ell}} \alpha_{\ell-1}}\left(Y_{\ell-1}^{\#}\right)^{[\alpha, \ell-1]}\left(\alpha_{\ell-1}\right)\left(\left(Y_{\ell}^{\#}\right)^{[\alpha, \ell]}\left(\alpha_{\ell-1}\right)\right)^{-1}
\end{aligned}
$$

for each integer $\ell \in\{1,2, \ldots, \tau\}$. In particular, the matrices

$$
\begin{aligned}
& \frac{\eta_{\ell} \overline{\eta_{\ell-1}}}{1-\overline{\alpha_{\ell}} \alpha_{\ell-1}}\left(\left(X_{\ell}^{\#}\right)^{[\alpha, \ell]}\left(\alpha_{\ell-1}\right)\right)^{-1}\left(X_{\ell-1}^{\#}\right)^{[\alpha, \ell-1]}\left(\alpha_{\ell-1}\right), \\
& \frac{\eta_{\ell} \overline{\eta_{\ell-1}}}{1-\overline{\alpha_{\ell}} \alpha_{\ell-1}}\left(Y_{\ell-1}^{\#}\right)^{[\alpha, \ell-1]}\left(\alpha_{\ell-1}\right)\left(\left(Y_{\ell}^{\#}\right)^{[\alpha, \ell]}\left(\alpha_{\ell-1}\right)\right)^{-1}
\end{aligned}
$$

are positive Hermitian for each integer $\ell \in\{1,2, \ldots, \tau\}$. Thus, the considerations at the 
first part of the proof yield that $\left[\left(X_{j}^{\#}\right)_{j=0}^{\tau},\left(Y_{j}^{\#}\right)_{j=0}^{\tau}\right]$ is the Szegö pair of rational matrixvalued functions generated by $\left[\left(\alpha_{j}\right)_{j=0}^{\tau} ;\left(-\mathbf{E}_{\ell}\right)_{\ell=1}^{\tau} ;\left(\mathbf{X}_{0}^{-1}\right)^{*},\left(\mathbf{Y}_{0}^{-1}\right)^{*}\right]$, or in other words, $\left[\left(X_{j}^{\#}\right)_{j=0}^{\tau},\left(Y_{j}^{\#}\right)_{j=0}^{\tau}\right]$ is the dual Szegö pair of $\left[\left(X_{j}\right)_{j=0}^{\tau},\left(Y_{j}\right)_{j=0}^{\tau}\right]$.

Remark that, in view of Corollary 3.3 (cf. (5.10)), it is not hard to accept that for an $\ell \in\{1,2, \ldots, \tau\}$ the definition of $\mathbf{E}_{\ell}$ in Theorem 5.4 can be replaced by

$$
\mathbf{E}_{\ell}:=\eta_{\ell} \overline{\eta_{\ell-1}} X_{\ell}\left(\alpha_{\ell-1}\right)\left(Y_{\ell}^{[\alpha, \ell]}\left(\alpha_{\ell-1}\right)\right)^{-1}
$$

and the left-hand side of the relation in the third line of (iii) by $X_{\ell}\left(\alpha_{\ell-1}\right)\left(Y_{\ell}^{[\alpha, \ell]}\left(\alpha_{\ell-1}\right)\right)^{-1}$ or the right-hand side by $-X_{\ell}^{\#}\left(\alpha_{\ell-1}\right)\left(\left(Y_{\ell}^{\#}\right)^{[\alpha, \ell]}\left(\alpha_{\ell-1}\right)\right)^{-1}$.

\section{On particular measures corresponding to the dual pairs}

We study now an inverse question to Theorem 2.2. By using similar arguments as in [26, Section 3], it is not hard to accept that if $\left[\left(X_{j}\right)_{j=0}^{\tau},\left(Y_{j}\right)_{j=0}^{\tau}\right]$ is some Szegö pair of rational matrix-valued functions, then there exists a nonnegative Hermitian $q \times q$ Borel measure $F$ on $\mathbb{T}$ so that $\left[\left(X_{j}\right)_{j=0}^{\tau},\left(Y_{j}\right)_{j=0}^{\tau}\right]$ is exactly a Szegö pair of orthonormal systems corresponding to $\left(\alpha_{j}\right)_{j=0}^{\tau}$ and $F$. The following considerations are to explain that the construction of such a measure occurring already in [26, Section 3] includes actually a simultaneous answer referring to the dual pair $\left[\left(X_{j}^{\#}\right)_{j=0}^{\tau},\left(Y_{j}^{\#}\right)_{j=0}^{\tau}\right]$ of $\left[\left(X_{j}\right)_{j=0}^{\tau},\left(Y_{j}\right)_{j=0}^{\tau}\right]$. For technical reasons we prove before two useful results. Here and in the sequel, if $\mathbf{A}$ is a complex $q \times q$ matrix, then $\mathfrak{R e} \mathbf{A}$ stands for the real part of $\mathbf{A}$, that is, $\mathfrak{R e} \mathbf{A}:=(1 / 2)\left(\mathbf{A}+\mathbf{A}^{*}\right)$.

Lemma 6.1. For each integer $j \in\{0,1, \ldots, \tau\}$ and point $z \in \mathbb{T}$,

$$
\begin{aligned}
& \frac{1-\left|\alpha_{j}\right|^{2}}{\left|z-\alpha_{j}\right|^{2}}\left(X_{j}(z)\right)^{-1}\left(\left(X_{j}(z)\right)^{-1}\right)^{*} \\
& \quad=\mathfrak{R e}\left[\left(X_{j}^{\#}\right)^{[\alpha, j]}(z)\left(X_{j}^{[\alpha, j]}(z)\right)^{-1}\right]=\mathfrak{R e}\left[\left(Y_{j}^{[\alpha, j]}(z)\right)^{-1}\left(Y_{j}^{\#}\right)^{[\alpha, j]}(z)\right] \\
& =\frac{1-\left|\alpha_{j}\right|^{2}}{\left|z-\alpha_{j}\right|^{2}}\left(\left(Y_{j}(z)\right)^{-1}\right)^{*}\left(Y_{j}(z)\right)^{-1}
\end{aligned}
$$

if $\left[\left(X_{j}\right)_{j=0}^{\tau},\left(Y_{j}\right)_{j=0}^{\tau}\right]$ is a Szegö pair of rational matrix-valued functions generated by some $\left[\left(\alpha_{j}\right)_{j=0}^{\tau} ;\left(\mathbf{E}_{\ell}\right)_{\ell=1}^{\tau} ; \mathbf{X}_{0}, \mathbf{Y}_{0}\right]$, where $\left(\mathbf{E}_{\ell}\right)_{\ell=1}^{\tau}$ is a sequence of strictly contractive $q \times q$ matrices and $\mathbf{X}_{0}, \mathbf{Y}_{0}$ are nonsingular $q \times q$ matrices fulfilling $\mathbf{X}_{0}^{*} \mathbf{X}_{0}=\mathbf{Y}_{0} \mathbf{Y}_{0}^{*}$.

Proof. Let $j \in\{0,1, \ldots, \tau\}$ and $z \in \mathbb{T}$. In view of Corollary 3.2 and (2.1) it follows that the matrices $X_{j}^{[\alpha, j]}(z), Y_{j}^{[\alpha, j]}(z), X_{j}(z)$, and $Y_{j}(z)$ are nonsingular. Therefore, an application of Corollary 3.7 yields

$$
\mathfrak{R e}\left[\left(X_{j}^{\#}\right)^{[\alpha, j]}(z)\left(X_{j}^{[\alpha, j]}(z)\right)^{-1}\right]=\mathfrak{R} \mathfrak{e}\left[\left(Y_{j}^{[\alpha, j]}(z)\right)^{-1}\left(Y_{j}^{\#}\right)^{[\alpha, j]}(z)\right]
$$


as well as by virtue of $(2.1), z=1 / \bar{z}$, and $\left|B_{j}(z)\right|^{2}=1$,

$$
\begin{aligned}
& \frac{1-\left|\alpha_{j}\right|^{2}}{\left|z-\alpha_{j}\right|^{2}}\left(X_{j}(z)\right)^{-1}\left(\left(X_{j}(z)\right)^{-1}\right)^{*} \\
& =\frac{1-\left|\alpha_{j}\right|^{2}}{\left|z-\alpha_{j}\right|^{2}}\left(\left(X_{j}^{[\alpha, j]}(z)\right)^{-1}\right)^{*}\left(X_{j}^{[\alpha, j]}(z)\right)^{-1} \\
& =\frac{1-\overline{\alpha_{j}} z}{-2 \eta_{j}\left(1-\alpha_{j} \bar{z}\right)} \overline{B_{j}(z)}\left(\left(X_{j}^{[\alpha, j]}(z)\right)^{-1}\right)^{*}\left(-2 \eta_{j} \frac{1-\left|\alpha_{j}\right|^{2}}{\left(1-\overline{\alpha_{j}} z\right)^{2}} B_{j}(z) \mathbf{I}_{q}\right)\left(X_{j}^{[\alpha, j]}(z)\right)^{-1} \\
& =\frac{1}{2} z \overline{B_{j}(z)}\left(\left(X_{j}^{[\alpha, j]}(z)\right)^{-1}\right)^{*}\left(X_{j}(z)\left(X_{j}^{\#}\right)^{[\alpha, j]}(z)+X_{j}^{\#}(z) X_{j}^{[\alpha, j]}(z)\right)\left(X_{j}^{[\alpha, j]}(z)\right)^{-1} \\
& =\frac{1}{2}\left(\left(X_{j}^{[\alpha, j]}(z)\right)^{-1}\right)^{*}\left(\left(X_{j}^{[\alpha, j]}(z)\right)^{*}\left(X_{j}^{\#}\right)^{[\alpha, j]}(z)+\left(\left(X_{j}^{\#}\right)^{[\alpha, j]}(z)\right)^{*} X_{j}^{[\alpha, j]}(z)\right)\left(X_{j}^{[\alpha, j]}(z)\right)^{-1} \\
& =\frac{1}{2}\left(\left(X_{j}^{\#}\right)^{[\alpha, j]}(z)\left(X_{j}^{[\alpha, j]}(z)\right)^{-1}+\left(\left(X_{j}^{\#}\right)^{[\alpha, j]}(z)\left(X_{j}^{[\alpha, j]}(z)\right)^{-1}\right)^{*}\right) \\
& =\mathfrak{R e}\left[\left(X_{j}^{\#}\right)^{[\alpha, j]}(z)\left(X_{j}^{[\alpha, j]}(z)\right)^{-1}\right]
\end{aligned}
$$

and similarly

$$
\frac{1-\left|\alpha_{j}\right|^{2}}{\left|z-\alpha_{j}\right|^{2}}\left(\left(Y_{j}(z)\right)^{-1}\right)^{*}\left(Y_{j}(z)\right)^{-1}=\mathfrak{R e}\left[\left(Y_{j}^{[\alpha, j]}(z)\right)^{-1}\left(Y_{j}^{\#}\right)^{[\alpha, j]}(z)\right] \text {. }
$$

Lemma 6.2. If $\tilde{X}_{n}, \tilde{Y}_{n} \in \breve{\mathscr{R}}_{\alpha, n}^{q \times q}$, then there is at most one Szegö pair of rational matrix-valued functions $\left[\left(X_{j}\right)_{j=0}^{n},\left(Y_{j}\right)_{j=0}^{n}\right]$ generated by some $\left[\left(\alpha_{j}\right)_{j=0}^{n} ;\left(\mathbf{E}_{\ell}\right)_{\ell=1}^{n} ; \mathbf{X}_{0}, \mathbf{Y}_{0}\right]$ such that $X_{n}=\tilde{X}_{n}$ and $Y_{n}=\tilde{Y}_{n}$, where $\left(\mathbf{E}_{\ell}\right)_{\ell=1}^{n}$ is a sequence of strictly contractive $q \times q$ matrices and $\mathbf{X}_{0}, \mathbf{Y}_{0}$ are nonsingular $q \times q$ matrices fulfilling $\mathbf{X}_{0}^{*} \mathbf{X}_{0}=\mathbf{Y}_{0} \mathbf{Y}_{0}^{*}$.

Proof. In the case $n=0$, the assertion follows directly from (2.4). Since Corollary 3.3 and (2.1) show that if $\left[\left(X_{j}\right)_{j=0}^{n},\left(Y_{j}\right)_{j=0}^{n}\right]$ is the Szegö pair of rational matrix-valued functions generated by some $\left[\left(\alpha_{j}\right)_{j=0}^{n} ;\left(\mathbf{E}_{\ell}\right)_{\ell=1}^{n} ; \mathbf{X}_{0}, \mathbf{Y}_{0}\right]$, then for each integer $\ell \in\{1,2, \ldots, n\}$ the parameter $\mathbf{E}_{\ell}$ can be recovered via the elements $X_{\ell}$ and $Y_{\ell}$ and since Proposition 2.4 and (2.1) yield that the elements $X_{\ell-1}$ and $Y_{\ell-1}$ are uniquely determined by $X_{\ell}, Y_{\ell}$, and $\mathbf{E}_{\ell}$, by induction on $n$, one can finally conclude that the assertion is also fulfilled if $n$ is a positive integer.

Remark that, by virtue of a well-known matricial version of a theorem due to RieszHerglotz (see, e.g., [11, Theorem 2.2.2]), there is a correspondence between nonnegative Hermitian Borel measures on $\mathbb{T}$ and matrix-valued Carathéodory functions. Recall that a $q \times q$ Carathéodory function $($ in $\mathbb{D}$ ) is a matrix function $\Omega$ from $\mathbb{D}$ into the set of complex $q \times q$ matrices which is holomorphic in $\mathbb{D}$ and which has a nonnegative Hermitian real part $\mathfrak{R} \mathfrak{e} \Omega(w)$ for each $w \in \mathbb{D}$. In particular, if $\Omega$ is a $q \times q$ Carathéodory function, then 
there is a unique nonnegative Hermitian $q \times q$ Borel measure $F$ on $\mathbb{T}$ such that

$$
\Omega(w)-\mathrm{i} \mathfrak{J m} \Omega(0)=\int_{\mathbb{T}} \frac{z+w}{z-w} F(\mathrm{~d} z), \quad w \in \mathbb{D},
$$

where $\mathfrak{I m} \Omega(0)$ stands for the imaginary part of the complex $q \times q$ matrix $\Omega(0)$, that is, $\mathfrak{J} \mathfrak{m} \Omega(0):=(1 / 2 \mathrm{i})\left(\Omega(0)-(\Omega(0))^{*}\right)$. This unique measure $F$ is called the Riesz-Herglotz measure associated with $\Omega$.

In the following, the notation $\lambda$ stands for the (ordinary) linear Lebesgue Borel measure on $\mathbb{T}$ (not normalized, i.e., $\lambda(\mathbb{T})=2 \pi$ ).

Theorem 6.3. Let $\left[\left(X_{j}\right)_{j=0}^{n},\left(Y_{j}\right)_{j=0}^{n}\right]$ be the Szegö pair of rational matrix-valued functions generated by $\left[\left(\alpha_{j}\right)_{j=0}^{n} ;\left(\mathrm{E}_{\ell}\right)_{\ell=1}^{n} ; \mathbf{X}_{0}, \mathbf{Y}_{0}\right]$ for some nonnegative integer $n$, some sequence $\left(\mathbf{E}_{\ell}\right)_{\ell=1}^{n}$ of strictly contractive $q \times q$ matrices, and some nonsingular complex $q \times q$ matrices $\mathbf{X}_{0}, \mathbf{Y}_{0}$ fulfilling $\mathbf{X}_{0}^{*} \mathbf{X}_{0}=\mathbf{Y}_{0} \mathbf{Y}_{0}^{*}$. By the relation

$$
F_{n}(A):=\frac{1}{2 \pi} \int_{A} \frac{1-\left|\alpha_{n}\right|^{2}}{\left|z-\alpha_{n}\right|^{2}}\left(X_{n}(z)\right)^{-1}\left(\left(X_{n}(z)\right)^{-1}\right)^{*} \lambda(\mathrm{d} z), \quad A \in \mathfrak{B}_{\mathbb{T}},
$$

a nonnegative Hermitian $q \times q$ Borel measure on $\mathbb{T}$ is well defined, whereby

$$
F_{n}(A)=\frac{1}{2 \pi} \int_{A} \frac{1-\left|\alpha_{n}\right|^{2}}{\left|z-\alpha_{n}\right|^{2}}\left(\left(Y_{n}(z)\right)^{-1}\right)^{*}\left(Y_{n}(z)\right)^{-1} \lambda(\mathrm{d} z), \quad A \in \mathfrak{B}_{\mathbb{T}},
$$

and $\left[\left(X_{j}\right)_{j=0}^{n},\left(Y_{j}\right)_{j=0}^{n}\right]$ is a Szegö pair of orthonormal systems corresponding to $\left(\alpha_{j}\right)_{j=0}^{n}$ and $F_{n}$. Further, if $\left[\left(X_{j}^{\#}\right)_{j=0}^{n},\left(Y_{j}^{\#}\right)_{j=0}^{n}\right]$ is the dual Szegö pair of $\left[\left(X_{j}\right)_{j=0}^{n},\left(Y_{j}\right)_{j=0}^{n}\right]$, then

$$
\Omega_{n}(w):=\left(X_{n}^{\#}\right)^{[\alpha, n]}(w)\left(X_{n}^{[\alpha, n]}(w)\right)^{-1}, \quad w \in \mathbb{D},
$$

defines a $q \times q$ Carathéodory function, whereby

$$
\Omega_{n}(w)=\left(Y_{n}^{[\alpha, n]}(w)\right)^{-1}\left(Y_{n}^{\#}\right)^{[\alpha, n]}(w), \quad w \in \mathbb{D}
$$

and $F_{n}$ is the Riesz-Herglotz measure associated with $\Omega_{n}$.

Proof. From Lemma 6.1 one can particularly see that the measure $F_{n}$ is well defined and that $F_{n}$ admits also the second representation. Moreover, Corollary 3.2 implies that $\Omega_{n}$ is well defined and actually that $\Omega_{n}$ is a $q \times q$ Carathéodory function. In combination with Corollary 3.7 we obtain then the second description of $\Omega_{n}$. Since property (I) of adjoint rational functions and Corollary 3.2 show that the function $\left(X_{n}^{\#}\right)^{[\alpha, n]}\left(X_{n}^{[\alpha, n]}\right)^{-1}$ is holomorphic in a disk enclosing $\mathbb{T}$, an application of Poisson's formula yields

$$
\begin{aligned}
\Omega_{n}(w) & =\left(X_{n}^{\#}\right)^{[\alpha, n]}(w)\left(X_{n}^{[\alpha, n]}(w)\right)^{-1} \\
& =\frac{1}{2 \pi} \int_{\mathbb{T}} \frac{1-|w|^{2}}{|z-w|^{2}}\left(X_{n}^{\#}\right)^{[\alpha, n]}(z)\left(X_{n}^{[\alpha, n]}(z)\right)^{-1} \lambda(\mathrm{d} z), \quad w \in \mathbb{D} .
\end{aligned}
$$


Hence, from Lemma 6.1 and

$$
\frac{1-|w|^{2}}{|z-w|^{2}}=\mathfrak{R e}\left[\frac{z+w}{z-w}\right], \quad z \in \mathbb{T}
$$

it follows that

$$
\begin{aligned}
\mathfrak{R e} \Omega_{n}(w) & =\frac{1}{2 \pi} \int_{\mathbb{T}} \frac{1-|w|^{2}}{|z-w|^{2}} \mathfrak{R e}\left[\left(X_{n}^{\#}\right)^{[\alpha, n]}(z)\left(X_{n}^{[\alpha, n]}(z)\right)^{-1}\right] \lambda(\mathrm{d} z) \\
& =\frac{1}{2 \pi} \int_{\mathbb{T}} \frac{1-|w|^{2}}{|z-w|^{2}} \frac{1-\left|\alpha_{n}\right|^{2}}{\left|z-\alpha_{n}\right|^{2}}\left(X_{n}(z)\right)^{-1}\left(\left(X_{n}(z)\right)^{-1}\right)^{*} \lambda(\mathrm{d} z) \\
& =\frac{1}{2 \pi} \int_{\mathbb{T}} \mathfrak{R e}\left[\frac{z+w}{z-w}\right] \frac{1-\left|\alpha_{n}\right|^{2}}{\left|z-\alpha_{n}\right|^{2}}\left(X_{n}(z)\right)^{-1}\left(\left(X_{n}(z)\right)^{-1}\right)^{*} \lambda(\mathrm{d} z) \\
& =\mathfrak{R e}\left[\frac{1}{2 \pi} \int_{\mathbb{T}}\left(\frac{z+w}{z-w}\right) \frac{1-\left|\alpha_{n}\right|^{2}}{\left|z-\alpha_{n}\right|^{2}}\left(X_{n}(z)\right)^{-1}\left(\left(X_{n}(z)\right)^{-1}\right)^{*} \lambda(\mathrm{d} z)\right] \\
& =\mathfrak{R e}\left[\int_{\mathbb{T}} \frac{z+w}{z-w} F_{n}(\mathrm{~d} z)\right], \quad w \in \mathbb{D} .
\end{aligned}
$$

Therefore, because $\Omega_{n}$ is holomorphic in $\mathbb{D}$ and also by

$$
\Omega_{F_{n}}(w):=\int_{\mathbb{T}} \frac{z+w}{z-w} F_{n}(\mathrm{~d} z), \quad w \in \mathbb{D},
$$

a holomorphic function in $\mathbb{D}$ is defined (see, e.g., [11, Theorem 2.2.2]), the Cauchy-Riemann differential equations imply that

$$
\Omega_{n}(w)=\Omega_{F_{n}}(w)+\mathrm{i} \mathbf{H}, \quad w \in \mathbb{D},
$$

for some Hermitian $q \times q$ matrix $\mathbf{H}$, that is, $F_{n}$ is the Riesz-Herglotz measure associated with $\Omega_{n}$. By virtue of the definition of $F_{n}$ (cf. [23, Example 5.4], [25, Corollary 4.4], and $\left[26\right.$, Remark 2.2]) there exists a Szegö pair of orthonormal systems $\left[\left(\tilde{X}_{j}\right)_{j=0}^{n},\left(\tilde{Y}_{j}\right)_{j=0}^{n}\right]$ corresponding to $\left(\alpha_{j}\right)_{j=0}^{n}$ and $F_{n}$. Let $j \in\{0,1, \ldots, n\}$. Since properties (I), (II) of adjoint rational matrix-valued functions and Corollary 3.2 show that the function $\left(X_{n}^{[\alpha, n]}\right)^{-1} X_{j}^{[\alpha, n]}$ is holomorphic in a disk enclosing $\mathbb{T}$ and that the identity $\left(X_{n}^{[\alpha, n]}\right)^{-1}\left(\alpha_{n}\right) X_{j}^{[\alpha, n]}\left(\alpha_{n}\right)=\delta_{n j} \mathbf{I}_{q}$ is fulfilled, an application of Poisson's formula yields

$$
\begin{aligned}
\int_{\mathbb{T}} X_{n}(z) F_{n}(\mathrm{~d} z)\left(X_{j}(z)\right)^{*} \\
=\frac{1}{2 \pi} \int_{\mathbb{T}} \frac{1-\left|\alpha_{n}\right|^{2}}{\left|z-\alpha_{n}\right|^{2}} X_{n}(z)\left(X_{n}(z)\right)^{-1}\left(\left(X_{n}(z)\right)^{-1}\right)^{*}\left(X_{j}(z)\right)^{*} \lambda(\mathrm{d} z) \\
=\frac{1}{2 \pi} \int_{\mathbb{T}} \frac{1-\left|\alpha_{n}\right|^{2}}{\left|z-\alpha_{n}\right|^{2}}\left(\left(X_{n}\left(\frac{1}{\bar{z}}\right)\right)^{-1}\right)^{*}\left(X_{j}\left(\frac{1}{\bar{z}}\right)\right)^{*} \lambda(\mathrm{d} z) \\
=\frac{1}{2 \pi} \int_{\mathbb{T}} \frac{1-\left|\alpha_{n}\right|^{2}}{\left|z-\alpha_{n}\right|^{2}}\left(X_{n}^{[\alpha, n]}(z)\right)^{-1} X_{j}^{[\alpha, n]}(z) \lambda(\mathrm{d} z)=\delta_{n j} \mathbf{I}_{q} .
\end{aligned}
$$


Similarly, using the second description of $F_{n}$ and Poisson's formula we obtain

$$
\int_{\mathbb{T}}\left(Y_{j}(z)\right)^{*} F_{n}(\mathrm{~d} z) Y_{n}(z)=\delta_{j n} \mathbf{I}_{q} .
$$

Thus (cf. [25, Remark 3.1 and Lemma 3.2] and note Remark 3.4), there exist some unitary $q \times q$ matrices $\mathbf{U}$ and $\mathbf{V}$ such that the equalities $X_{n}=\mathbf{U} \tilde{X}_{n}$ and $Y_{n}=\tilde{Y}_{n} \mathbf{V}$ are satisfied. Since the choice of $\left[\left(\tilde{X}_{j}\right)_{j=0}^{n},\left(\tilde{Y}_{j}\right)_{j=0}^{n}\right]$ and Theorem 2.2 (note also [26, Remark 2.3]) imply that $\left[\left(\mathbf{U} \tilde{X}_{j}\right)_{j=0}^{n},\left(\tilde{Y}_{j} \mathbf{V}\right)_{j=0}^{n}\right]$ is the Szegö pair of rational matrix-valued functions generated by $\left[\left(\alpha_{j}\right)_{j=0}^{n} ;\left(\mathbf{U} \widetilde{E}_{\ell} \mathbf{V}\right)_{\ell=1}^{n} ; \mathbf{U} \tilde{\mathbf{X}}_{0}, \tilde{\mathbf{Y}}_{0} \mathbf{V}\right]$, where

$$
\widetilde{\mathbf{E}}_{\ell}:=\eta_{\ell} \overline{\eta_{\ell-1}}\left(\tilde{X}_{\ell}^{[\alpha, \ell]}\left(\alpha_{\ell-1}\right)\right)^{-1} \tilde{Y}_{\ell}\left(\alpha_{\ell-1}\right), \quad \ell \in\{1,2, \ldots, n\}
$$

$\tilde{\mathbf{X}}_{0}:=\sqrt{1-\left|\alpha_{0}\right|^{2}} \tilde{X}_{0}\left(\alpha_{0}\right)$, and $\tilde{\mathbf{Y}}_{0}:=\sqrt{1-\left|\alpha_{0}\right|^{2}} \tilde{Y}_{0}\left(\alpha_{0}\right)$, from Lemma 6.2 we can conclude that $X_{j}=\mathbf{U} \tilde{X}_{j}$ and $Y_{j}=\tilde{Y}_{j} \mathbf{V}$ for each $j \in\{0,1, \ldots, n\}$. Hence,

$$
\begin{aligned}
& \int_{\mathbb{T}} X_{j}(z) F_{n}(\mathrm{~d} z)\left(X_{k}(z)\right)^{*}=\mathbf{U}\left(\int_{\mathbb{T}} \tilde{X}_{j}(z) F_{n}(\mathrm{~d} z)\left(\tilde{X}_{k}(z)\right)^{*}\right) \mathbf{U}^{*}=\delta_{j k} \mathbf{U U}^{*}=\delta_{j k} \mathbf{I}_{q}, \\
& \int_{\mathbb{T}}\left(Y_{j}(z)\right)^{*} F_{n}(\mathrm{~d} z) Y_{k}(z)=\mathbf{V}^{*}\left(\int_{\mathbb{T}}\left(\tilde{Y}_{j}(z)\right)^{*} F_{n}(\mathrm{~d} z) \tilde{Y}_{k}(z)\right) \mathbf{V}=\delta_{j k} \mathbf{V}^{*} \mathbf{V}=\delta_{j k} \mathbf{I}_{q}
\end{aligned}
$$

for each $j, k \in\{0,1, \ldots, n\}$. Finally, Corollary 3.3 yields that the matrices

$$
\frac{\eta_{\ell} \overline{\eta_{\ell-1}}}{1-\overline{\alpha_{\ell}} \alpha_{\ell-1}}\left(X_{\ell}^{[\alpha, \ell]}\left(\alpha_{\ell-1}\right)\right)^{-1} X_{\ell-1}^{[\alpha, \ell-1]}\left(\alpha_{\ell-1}\right), \quad \frac{\eta_{\ell} \overline{\eta_{\ell-1}}}{1-\overline{\alpha_{\ell}} \alpha_{\ell-1}} Y_{\ell-1}^{[\alpha, \ell-1]}\left(\alpha_{\ell-1}\right)\left(Y_{\ell}^{[\alpha, \ell]}\left(\alpha_{\ell-1}\right)\right)^{-1}
$$

are positive Hermitian for each $\ell \in\{1,2, \ldots, n\}$ such that $\left[\left(X_{j}\right)_{j=0}^{n},\left(Y_{j}\right)_{j=0}^{n}\right]$ is actually a Szegö pair of orthonormal systems corresponding to $\left(\alpha_{j}\right)_{j=0}^{n}$ and $F_{n}$.

Note that Theorem 6.3 can be considered in a sense as an extension of [6, Theorem 4.2.6 and Lemma 8.1.3] to the matrix case. Furthermore, an alternative proof of a matricial version of [6, Lemma 8.1.3] is given in [26, Theorem 3.5].

Example 6.4. If $\tilde{X}_{n} \in \breve{\mathscr{R}}_{\alpha, n}^{q \times q}$ (resp., $\tilde{Y}_{n} \in \breve{\mathscr{R}}_{\alpha, n}^{q \times q}$ ) fulfilling the condition $\operatorname{det} \widetilde{X}_{n}^{[\alpha, n]}(u) \neq 0$ (resp., $\operatorname{det} \tilde{Y}_{n}^{[\alpha, n]}(u) \neq 0$ ) for each $u \in \mathbb{D} \cup \mathbb{T}$, then similar as performed in [27, Lemmas 4.1 and 4.2 ], there exists a left (resp., right) orthonormal system $\left(Z_{j}\right)_{j=0}^{n}$ corresponding to $\left(\alpha_{j}\right)_{j=0}^{n}$ and the nonnegative Hermitian $q \times q$ Borel measure on $\mathbb{T}$ which is given, for each $A \in \mathfrak{B}_{\mathbb{T}}$, by the relation

$$
\begin{gathered}
F_{L}(A):=\frac{1}{2 \pi} \int_{A} \frac{1-\left|\alpha_{n}\right|^{2}}{\left|z-\alpha_{n}\right|^{2}}\left(\tilde{X}_{n}(z)\right)^{-1}\left(\left(\tilde{X}_{n}(z)\right)^{-1}\right)^{*} \lambda(\mathrm{d} z) \\
\left(\operatorname{resp} ., F_{R}(A):=\frac{1}{2 \pi} \int_{A} \frac{1-\left|\alpha_{n}\right|^{2}}{\left|z-\alpha_{n}\right|^{2}}\left(\left(\tilde{Y}_{n}(z)\right)^{-1}\right)^{*}\left(\tilde{Y}_{n}(z)\right)^{-1} \lambda(\mathrm{d} z)\right)
\end{gathered}
$$


such that $Z_{n}=\tilde{X}_{n}$ (resp., $Z_{n}=\tilde{Y}_{n}$ ). In particular, if for each $z \in \mathbb{T}$ the additional condition $\left(\tilde{X}_{n}(z)\right) * \tilde{X}_{n}(z)=\tilde{Y}_{n}(z)\left(\tilde{Y}_{n}(z)\right) *$ is satisfied, then $F_{L}=F_{R}$ and, in view of [26, Remarks 2.2 and 2.3], Theorem 2.2, and Lemma 6.2, there is exactly one Szegö pair $\left[\left(X_{j}\right)_{j=0}^{n},\left(Y_{j}\right)_{j=0}^{n}\right]$ generated by some $\left[\left(\alpha_{j}\right)_{j=0}^{n} ;\left(\mathbf{E}_{\ell}\right)_{\ell=1}^{n} ; \mathbf{X}_{0}, \mathbf{Y}_{0}\right]$ such that $X_{n}=\tilde{X}_{n}$ and $Y_{n}=\tilde{Y}_{n}$. Moreover, Theorem 6.3 implies that

$$
\Omega(w):=\int_{\mathbb{T}} \frac{z+w}{z-w} F_{L}(\mathrm{~d} z), \quad w \in \mathbb{D},
$$

defines a $q \times q$ Carathéodory function which is the restriction of a rational matrix-valued function onto $\mathbb{D}$, where the elements $X_{n}^{\#}$ and $Y_{n}^{\#}$ of the dual Szegö pair $\left[\left(X_{j}^{\#}\right)_{j=0}^{n},\left(Y_{j}^{\#}\right)_{j=0}^{n}\right]$ of $\left[\left(X_{j}\right)_{j=0}^{n},\left(Y_{j}\right)_{j=0}^{n}\right]$ can be used to compute $\Omega$ or vice versa.

Due to Remark 2.1, from Theorem 6.3 one can conclude directly the following.

Corollary 6.5. Let $\left[\left(X_{j}\right)_{j=0}^{n},\left(Y_{j}\right)_{j=0}^{n}\right]$ be the Szegö pair of rational matrix-valued functions generated by $\left[\left(\alpha_{j}\right)_{j=0}^{n} ;\left(\mathbf{E}_{\ell}\right)_{\ell=1}^{n} ; \mathbf{X}_{0}, \mathbf{Y}_{0}\right]$ for some nonnegative integer $n$, some sequence $\left(\mathbf{E}_{\ell}\right)_{\ell=1}^{n}$ of strictly contractive $q \times q$ matrices, and some nonsingular complex $q \times q$ matrices $\mathbf{X}_{0}, \mathbf{Y}_{0}$ fulfilling the condition $\mathbf{X}_{0}^{*} \mathbf{X}_{0}=\mathbf{Y}_{0} \mathbf{Y}_{0}^{*}$. Furthermore, let $\left[\left(X_{j}^{\#}\right)_{j=0}^{n},\left(Y_{j}^{\#}\right)_{j=0}^{n}\right]$ be the dual Szegö pair of $\left[\left(X_{j}\right)_{j=0}^{n},\left(Y_{j}\right)_{j=0}^{n}\right]$. By

$$
F_{n}^{\#}(A):=\frac{1}{2 \pi} \int_{A} \frac{1-\left|\alpha_{n}\right|^{2}}{\left|z-\alpha_{n}\right|^{2}}\left(X_{n}^{\#}(z)\right)^{-1}\left(\left(X_{n}^{\#}(z)\right)^{-1}\right)^{*} \lambda(\mathrm{d} z), \quad A \in \mathfrak{B}_{\mathbb{T}},
$$

a nonnegative Hermitian $q \times q$ Borel measure on $\mathbb{T}$ is well defined, whereby

$$
F_{n}^{\#}(A)=\frac{1}{2 \pi} \int_{A} \frac{1-\left|\alpha_{n}\right|^{2}}{\left|z-\alpha_{n}\right|^{2}}\left(\left(Y_{n}^{\#}(z)\right)^{-1}\right)^{*}\left(Y_{n}^{\#}(z)\right)^{-1} \lambda(\mathrm{d} z), \quad A \in \mathfrak{B}_{\mathbb{T}},
$$

and $\left[\left(X_{j}^{\#}\right)_{j=0}^{n},\left(Y_{j}^{\#}\right)_{j=0}^{n}\right]$ is a Szegö pair of orthonormal systems corresponding to $\left(\alpha_{j}\right)_{j=0}^{n}$ and $F_{n}^{\#}$. Moreover,

$$
\Omega_{n}^{\#}(w):=X_{n}^{[\alpha, n]}(w)\left(\left(X_{n}^{\#}\right)^{[\alpha, n]}(w)\right)^{-1}, \quad w \in \mathbb{D},
$$

defines a $q \times q$ Carathéodory function, whereby

$$
\Omega_{n}^{\#}(w)=\left(\left(Y_{n}^{\#}\right)^{[\alpha, n]}(w)\right)^{-1} Y_{n}^{[\alpha, n]}(w), \quad w \in \mathbb{D},
$$

and $F_{n}^{\#}$ is the Riesz-Herglotz measure associated with $\Omega_{n}^{\#}$.

Remark 6.6. The interrelation between the $q \times q$ Carathéodory functions occurring in Theorem 6.3 and Corollary 6.5 is given by the equality $\Omega_{n}^{\#}(w)=\left(\Omega_{n}(w)\right)^{-1}, w \in \mathbb{D}$. Hence, in view of the notion given in [11, Definition 3.6.10], the nonnegative Hermitian $q \times q$ Borel measure $F_{n}^{\#}$ defined in Corollary 6.5 is the reciprocal measure corresponding to the measure $F_{n}$ defined in Theorem 6.3 if and only if the Hermitian $q \times q$ matrix $\mathbf{H}$ that appears in the proof of Theorem 6.3 is equal to the zero matrix $\mathbf{0}_{q}$. In particular, if $\alpha_{0}=0$, then Corollary 3.10 yields $\mathbf{H}=\mathbf{0}_{q}$. 
By using some well-known results on weak convergence of nonnegative Hermitian $q \times q$ Borel measures (see, e.g., [20]) we study now a little the case of Szegö pairs of infinite sequences of rational matrix-valued functions. Note that one says that a sequence $\left(F_{n}\right)_{n=0}^{\infty}$ of nonnegative Hermitian $q \times q$ Borel measures on $\mathbb{T}$ converges weakly to a nonnegative Hermitian $q \times q$ Borel measure $F$ on $\mathbb{T}$ if

$$
\lim _{n \rightarrow \infty} \int_{\mathbb{T}} h F_{n}(\mathrm{~d} z)=\int_{\mathbb{T}} h F(\mathrm{~d} z)
$$

for each bounded, continuous, and real-valued function $h$ on $\mathbb{T}$.

Theorem 6.7. Let $\left[\left(X_{j}\right)_{j=0}^{\infty},\left(Y_{j}\right)_{j=0}^{\infty}\right]$ be the Szegö pair of rational matrix-valued functions generated by $\left[\left(\alpha_{j}\right)_{j=0}^{\infty} ;\left(\mathbf{E}_{\ell}\right)_{\ell=1}^{\infty} ; \mathbf{X}_{0}, \mathbf{Y}_{0}\right]$ and let $\left[\left(X_{j}^{\#}\right)_{j=0}^{\infty},\left(Y_{j}^{\#}\right)_{j=0}^{\infty}\right]$ be the dual Szegö pair of $\left[\left(X_{j}\right)_{j=0}^{\infty},\left(Y_{j}\right)_{j=0}^{\infty}\right]$ for some sequence $\left(\mathbf{E}_{\ell}\right)_{\ell=1}^{\infty}$ of strictly contractive $q \times q$ matrices and nonsingular complex $q \times q$ matrices $\mathbf{X}_{0}, \mathbf{Y}_{0}$ fulfilling $\mathbf{X}_{0}^{*} \mathbf{X}_{0}=\mathbf{Y}_{0} \mathbf{Y}_{0}^{*}$. If $F_{n}, \Omega_{n}$ and $F_{n}^{\#}, \Omega_{n}^{\#}$ are given as in Theorem 6.3 and Corollary 6.5 for each nonnegative integer $n$, then there exist a subsequence $\left(F_{n_{m}}\right)_{m=0}^{\infty}$ of $\left(F_{n}\right)_{n=0}^{\infty}$, a subsequence $\left(F_{n_{m}}^{\#}\right)_{m=0}^{\infty}$ of $\left(F_{n}^{\#}\right)_{n=0}^{\infty}$, and some nonnegative Hermitian $q \times q$ Borel measures $F, F^{\#}$ so that $\left(F_{n_{m}}\right)_{m=0}^{\infty}$ converges weakly to $F$ and $\left(F_{n_{m}}^{\#}\right)_{m=0}^{\infty}$ converges weakly to $F^{\#}$. In particular, $\left[\left(X_{j}\right)_{j=0}^{\infty},\left(Y_{j}\right)_{j=0}^{\infty}\right]$ is a Szegö pair of orthonormal systems corresponding to $\left(\alpha_{j}\right)_{j=0}^{\infty}$ and $F,\left[\left(X_{j}^{\#}\right)_{j=0}^{\infty},\left(Y_{j}^{\#}\right)_{j=0}^{\infty}\right]$ is a Szegö pair of orthonormal systems corresponding to $\left(\alpha_{j}\right)_{j=0}^{\infty}$ and $F^{\#}$, and

$$
\Omega(w):=\lim _{m \rightarrow \infty} \Omega_{n_{m}}(w), \quad \Omega^{\#}(w):=\lim _{m \rightarrow \infty} \Omega_{n_{m}}^{\#}(w), \quad w \in \mathbb{D},
$$

define $q \times q$ Carathéodory functions, whereby

$$
\Omega^{\#}(w)=(\Omega(w))^{-1}, \quad w \in \mathbb{D},
$$

$F$ is the Riesz-Herglotz measure associated with $\Omega$, and $F^{\#}$ is the Riesz-Herglotz measure associated with $\Omega^{\#}$.

Proof. Let $n$ be a nonnegative integer. Since

$$
\frac{1-\left|\alpha_{0}\right|}{1+\left|\alpha_{0}\right|} \leq \frac{1-\left|\alpha_{0}\right|^{2}}{\left|z-\alpha_{0}\right|^{2}}=\mathfrak{R e}\left[\frac{z+\alpha_{0}}{z-\alpha_{0}}\right], \quad z \in \mathbb{T},
$$

and since Theorem 6.3, Corollaries 3.10 and 6.5 imply

$$
\begin{aligned}
& \int_{\mathbb{T}} \mathfrak{R} \mathfrak{e}\left[\frac{z+\alpha_{0}}{z-\alpha_{0}}\right] F_{n}(\mathrm{~d} z)=\mathfrak{R} \mathfrak{e} \Omega_{n}\left(\alpha_{0}\right)=\Omega_{n}\left(\alpha_{0}\right)=\left(\mathbf{X}_{0}^{*} \mathbf{X}_{0}\right)^{-1}, \\
& \int_{\mathbb{T}} \mathfrak{R e}\left[\frac{z+\alpha_{0}}{z-\alpha_{0}}\right] F_{n}^{\#}(\mathrm{~d} z)=\mathfrak{R} \mathfrak{e} \Omega_{n}^{\#}\left(\alpha_{0}\right)=\left(\Omega_{n}\left(\alpha_{0}\right)\right)^{-1}=\mathbf{X}_{0}^{*} \mathbf{X}_{0},
\end{aligned}
$$

it follows that the sets $\left\{F_{0}(\mathbb{T}), F_{1}(\mathbb{T}), F_{2}(\mathbb{T}), \ldots\right\}$ and $\left\{F_{0}^{\#}(\mathbb{T}), F_{1}^{\#}(\mathbb{T}), F_{2}^{\#}(\mathbb{T}), \ldots\right\}$ are bounded. Therefore, a twofold application of a theorem due to Helly-Prohorov according to [11, Lemma 2.2.1] (see also [20, Satz 9]) provides the existence of some nonnegative 
Hermitian $q \times q$ Borel measures $F, F^{\#}$, a subsequence $\left(F_{n_{m}}\right)_{m=0}^{\infty}$ of $\left(F_{n}\right)_{n=0}^{\infty}$ which converges weakly to $F$, and a subsequence $\left(F_{n_{m}}^{\#}\right)_{m=0}^{\infty}$ of $\left(F_{n}^{\#}\right)_{n=0}^{\infty}$ which converges weakly to $F^{\#}$.

Theorem 6.3 yields that $\left[\left(X_{j}\right)_{j=0}^{n},\left(Y_{j}\right)_{j=0}^{n}\right]$ is a Szegö pair of orthonormal systems corresponding to $\left(\alpha_{j}\right)_{j=0}^{n}$ and $F_{n}$. Applying [20, Satz 3] we obtain for all nonnegative integers $j, k$ the equality

$$
\begin{aligned}
& \int_{\mathbb{T}} X_{j}(z) F(\mathrm{~d} z)\left(X_{k}(z)\right)^{*}=\lim _{m \rightarrow \infty} \int_{\mathbb{T}} X_{j}(z) F_{n_{m}}(\mathrm{~d} z)\left(X_{k}(z)\right)^{*}=\delta_{j k} \mathbf{I}_{q}, \\
& \int_{\mathbb{T}}\left(Y_{j}(z)\right)^{*} F(\mathrm{~d} z) Y_{k}(z)=\lim _{m \rightarrow \infty} \int_{\mathbb{T}}\left(Y_{j}(z)\right)^{*} F_{n_{m}}(\mathrm{~d} z) Y_{k}(z)=\delta_{j k} \mathbf{I}_{q},
\end{aligned}
$$

that is, $\left[\left(X_{j}\right)_{j=0}^{\infty},\left(Y_{j}\right)_{j=0}^{\infty}\right]$ is a pair of orthonormal systems corresponding to $\left(\alpha_{j}\right)_{j=0}^{\infty}$ and $F$. Corollary 3.3 yields that $\left[\left(X_{j}\right)_{j=0}^{\infty},\left(Y_{j}\right)_{j=0}^{\infty}\right]$ is actually a Szegö pair of orthonormal systems corresponding to $\left(\alpha_{j}\right)_{j=0}^{\infty}$ and F. Similarly, from Corollary 6.5, [20, Satz 3], and Corollary 3.3 one can conclude that $\left[\left(X_{j}^{\#}\right)_{j=0}^{\infty},\left(Y_{j}^{\#}\right)_{j=0}^{\infty}\right]$ is a Szegö pair of orthonormal systems corresponding to $\left(\alpha_{j}\right)_{j=0}^{\infty}$ and $F^{\#}$.

Now let $w \in \mathbb{D}$. Because

$$
h_{w}(z):=\frac{z+w}{z-w}, \quad z \in \mathbb{T},
$$

defines a bounded, continuous, and complex-valued function on $\mathbb{T}$, we get from Theorem 6.3, Corollary 3.10, and the choice of $F$ particularly

$$
\lim _{m \rightarrow \infty} i \mathfrak{J m} \Omega_{n_{m}}(0)=\lim _{m \rightarrow \infty} \Omega_{n_{m}}\left(\alpha_{0}\right)-\lim _{m \rightarrow \infty} \int_{\mathbb{T}} \frac{z+\alpha_{0}}{z-\alpha_{0}} F_{n_{m}}(\mathrm{~d} z)=\left(\mathbf{X}_{0}^{*} \mathbf{X}_{0}\right)^{-1}-\int_{\mathbb{T}} \frac{z+\alpha_{0}}{z-\alpha_{0}} F(\mathrm{~d} z),
$$

and hence

$$
\Omega(w)=\lim _{m \rightarrow \infty} \Omega_{n_{m}}(w)=\lim _{m \rightarrow \infty} \int_{\mathbb{T}} \frac{z+w}{z-w} F_{n_{m}}(\mathrm{~d} z)+\lim _{m \rightarrow \infty} \mathrm{i} \mathfrak{I m} \Omega_{n_{m}}(0)=\int_{\mathbb{T}} \frac{z+w}{z-w} F(\mathrm{~d} z)+\mathrm{i} \mathbf{H}
$$

for some Hermitian $q \times q$ matrix $\mathbf{H}$. Similarly, it follows that

$$
\Omega^{\#}(w)=\int_{\mathbb{T}} \frac{z+w}{z-w} F^{\#}(\mathrm{~d} z)+\mathrm{i} \mathbf{H}^{\#}
$$

for some Hermitian $q \times q$ matrix $\mathbf{H}^{\#}$. Consequently, $\Omega$ and $\Omega^{\#}$ are $q \times q$ Carathéodory functions, whereby $F$ is the Riesz-Herglotz measure associated with $\Omega$ and $F^{\#}$ is the RieszHerglotz measure associated with $\Omega^{\#}$. Finally, Corollary 3.10 provides

$$
\mathfrak{R e} \Omega\left(\alpha_{0}\right)=\lim _{m \rightarrow \infty} \mathfrak{R e} \Omega_{n_{m}}\left(\alpha_{0}\right)=\lim _{m \rightarrow \infty} \Omega_{n_{m}}\left(\alpha_{0}\right)=\left(\mathbf{X}_{0}^{*} \mathbf{X}_{0}\right)^{-1}
$$

such that [11, Proposition 2.1.3 (c) and Lemma 1.1.13 (c)] yield that the matrix $\Omega(w)$ is 
nonsingular, whereby in view of Remark 6.6 it follows that

$$
\Omega^{\#}(w)=\lim _{m \rightarrow \infty} \Omega_{n_{m}}^{\#}(w)=\lim _{m \rightarrow \infty}\left(\Omega_{n_{m}}(w)\right)^{-1}=(\Omega(w))^{-1} .
$$

Example 6.8. If $\left[\left(X_{j}\right)_{j=0}^{n},\left(Y_{j}\right)_{j=0}^{n}\right]$ is the Szegö pair of rational matrix-valued functions generated by $\left[\left(\alpha_{j}\right)_{j=0}^{n} ;\left(\mathbf{E}_{\ell}\right)_{\ell=1}^{n} ; \mathbf{X}_{0}, \mathbf{Y}_{0}\right]$ and if $\left[\left(X_{j}^{\#}\right)_{j=0}^{n},\left(Y_{j}^{\#}\right)_{j=0}^{n}\right]$ stands for its dual Szegö pair as in Theorem 6.3, then there is an obvious choice for parameters $\mathbf{E}_{n+1}, \mathbf{E}_{n+2}, \ldots$ such that $\left[\left(X_{j}\right)_{j=0}^{\infty},\left(Y_{j}\right)_{j=0}^{\infty}\right]$ is the Szegö pair of rational matrix-valued functions generated by $\left[\left(\alpha_{j}\right)_{j=0}^{\infty} ;\left(\mathbf{E}_{\ell}\right)_{\ell=1}^{\infty} ; \mathbf{X}_{0}, \mathbf{Y}_{0}\right]$ and $\left[\left(X_{j}^{\#}\right)_{j=0}^{\infty},\left(Y_{j}^{\#}\right)_{j=0}^{\infty}\right]$ is the dual Szegö pair of $\left[\left(X_{j}\right)_{j=0}^{\infty},\left(Y_{j}\right)_{j=0}^{\infty}\right]$, namely $\mathbf{E}_{\ell}=\mathbf{0}_{q}$ for each integer $\ell$ with $\ell>n$ (cf. Example 2.3). For this special situation, the construction of nonnegative Hermitian $q \times q$ Borel measures according to Theorem 6.3 and Corollary 6.5 implies the equalities $F_{\ell}=F_{n}$ and $F_{\ell}^{\#}=F_{n}^{\#}$ for each integer $\ell>n$ (cf. [26, Corollary 3.6]). Hence, Theorem 6.7 yields that $\left[\left(X_{j}\right)_{j=0}^{\infty},\left(Y_{j}\right)_{j=0}^{\infty}\right]$ is a Szegö pair of orthonormal systems corresponding to $\left(\alpha_{j}\right)_{j=0}^{\infty}$ and $F_{n}$ as well as $\left[\left(X_{j}^{\#}\right)_{j=0}^{\infty},\left(Y_{j}^{\#}\right)_{j=0}^{\infty}\right]$ is a Szegö pair of orthonormal systems corresponding to $\left(\alpha_{j}\right)_{j=0}^{\infty}$ and $F_{n}^{\#}$ in that case.

Finally, we remark that in opposition to the particular case of matrix polynomials on $\mathbb{T}$ (see, e.g., [11, Theorem 3.6.2]) a nonnegative Hermitian $q \times q$ Borel measure $F$ for which a Szegö pair $\left[\left(X_{j}\right)_{j=0}^{\infty},\left(Y_{j}\right)_{j=0}^{\infty}\right]$ of rational matrix-valued functions forms a Szegö pair of orthonormal systems corresponding to $\left(\alpha_{j}\right)_{j=0}^{\infty}$ and $F$ is not uniquely determined by $\left[\left(X_{j}\right)_{j=0}^{\infty},\left(Y_{j}\right)_{j=0}^{\infty}\right]$ in general. This phenomenon already arises in the case of complexvalued functions (see [6, Chapters 6-8] for details).

\section{Acknowledgments}

The work on the project which underlies the topic of this paper was supported by the German Academy of Natural Scientists Leopoldina via the Federal Ministry of Education and Research on badge BMBF-LPD 9901/8-88. Furthermore, the author wishes to express his gratitude to Professor B. Kirstein and Professor B. Fritzsche as well. Their interest, encouragement, and thought-provoking discussions were a valuable support.

\section{References}

[1] F. V. Atkinson, Discrete and Continuous Boundary Problems, Mathematics in Science and Engineering, vol. 8, Academic Press, New York, 1964.

[2] A. Blomqvist, A. Lindquist, and R. Nagamune, Matrix-valued Nevanlinna-Pick interpolation with complexity constraint: an optimization approach, IEEE Transactions on Automatic Control 48 (2003), no. 12, 2172-2190.

[3] A. Bultheel, P. González-Vera, E. Hendriksen, and O. Njåstad, A Szegö theory for rational functions, Report TW 131, Department of Computer Science, K.U., Leuven, 1990.

[4] _ Orthogonal rational functions and interpolatory product rules on the unit circle. I. Recurrence and interpolation, Analysis 18 (1998), no. 2, 167-183.

[5] __ Orthogonal rational functions and interpolatory product rules on the unit circle. II. Quadrature and convergence, Analysis 18 (1998), no. 2, 185-200.

[6] _ Orthogonal Rational Functions, Cambridge Monographs on Applied and Computational Mathematics, vol. 5, Cambridge University Press, Cambridge, 1999. 
[7] __ Orthogonal rational functions and interpolatory product rules on the unit circle. III. Convergence of general sequences, Analysis 20 (2000), no. 2, 99-120.

[8] G. Chen and Y. Hu, On the multiple Nevanlinna-Pick matrix interpolation in the class $\mathscr{b}_{p}$ and the Carathéodory matrix coefficient problem, Linear Algebra and Its Applications 283 (1998), no. 1-3, 179-203.

[9] P. Delsarte, Y. Genin, and Y. Kamp, Orthogonal polynomial matrices on the unit circle, IEEE Transactions on Circuits and Systems CAS-25 (1978), no. 3, 149-160.

[10] Schur parametrization of positive definite block-Toeplitz systems, SIAM Journal on Applied Mathematics 36 (1979), no. 1, 34-46.

[11] V. K. Dubovoj, B. Fritzsche, and B. Kirstein, Matricial Version of the Classical Schur Problem, Teubner-Texte zur Mathematik, vol. 129, B. G. Teubner, Stuttgart, 1992.

[12] H. Dym, J Contractive Matrix Functions, Reproducing Kernel Hilbert Spaces and Interpolation, CBMS Regional Conference Series in Mathematics, vol. 71, American Mathematical Society, Rhode Island, 1989.

[13] M. M. Džrbašjan, Orthogonal systems of rational functions on the circle with a prescribed set of poles, Doklady Akademii Nauk SSSR 147 (1962), 1278-1281 (Russian).

[14] A. V. Efimov and V. P. Potapov, J-expanding matrix-valued functions, and their role in the analytic theory of electrical circuits, Uspekhi Matematicheskikh Nauk 28 (1973), no. 1(169), 65-130 (Russian).

[15] C. Foias and A. E. Frazho, The Commutant Lifting Approach to Interpolation Problems, Operator Theory: Advances and Applications, vol. 44, Birkhäuser, Basel, 1990.

[16] B. Fritzsche, S. Fuchs, and B. Kirstein, An inverse entropy optimization problem for matrix-valued Carathéodory functions, Optimization 29 (1994), no. 1, 1-32.

[17] B. Fritzsche and B. Kirstein, An extension problem for nonnegative Hermitian block Toeplitz matrices, Mathematische Nachrichten 130 (1987), 121-135.

[18] _ An extension problem for nonnegative Hermitian block Toeplitz matrices. II, Mathematische Nachrichten 131 (1987), 287-297.

[19] __ An extension problem for nonnegative Hermitian block Toeplitz matrices. III, Mathematische Nachrichten 135 (1988), 319-341.

[20] _ Schwache Konvergenz nichtnegativ hermitescher Borelmaße, Wissenschaftliche Zeitschrift der Karl-Marx-Universität Leipzig. Mathematisch-Naturwissenschaftliche Reihe $\mathbf{3 7}$ (1988), no. 4, 375-398.

[21] _ An extension problem for nonnegative Hermitian block Toeplitz matrices. IV, Mathematische Nachrichten 143 (1989), 329-354.

[22] _ An extension problem for nonnegative Hermitian block Toeplitz matrices. $V$, Mathematische Nachrichten 144 (1989), 283-308.

[23] B. Fritzsche, B. Kirstein, and A. Lasarow, On rank invariance of moment matrices of nonnegative Hermitian-valued Borel measures on the unit circle, Mathematische Nachrichten 263/264 (2004), $103-132$.

[24] - On rank invariance of Schwarz-Pick-Potapov block matrices of matricial Schur functions, Integral Equations and Operator Theory 48 (2004), no. 3, 305-330.

[25] - Orthogonal rational matrix-valued functions on the unit circle, Mathematische Nachrichten 278 (2005), no. 5, 525-553.

[26] __ Szegö pairs of orthogonal rational matrix-valued functions on the unit circle, Operator Theory and Indefinite Inner Product Spaces, Operator Theory: Adv. and Appl., vol. 163, Birkhäuser, Basel, 2006, pp. 163-191.

[27]__ Orthogonal rational matrix-valued functions on the unit circle: recurrence relations and a Favard-type theorem, Mathematische Nachrichten 279, no. 5-6, 513-542. 
[28] P. A. Fuhrmann, Orthogonal matrix polynomials and system theory, Conference on Linear and Nonlinear Mathematical Control Theory, Rend. Sem. Mat. Univ. Politec., Torino, 1987, pp. 68124, special issue on Control Theory.

[29] Ja. L. Geronimus, On polynomials orthogonal on the circle, on trigonometric moment-problem and on allied Carathéodory and Schur functions, Mathematics of the USSR. Sbornik 15(57) (1944), 99-130 (Russian).

[30] On the trigonometric moment problem, Annals of Mathematics 47 (1946), 742-761.

[31]__ Orthogonal Polynomials, Fizmatgiz, Moskva, 1958.

[32] U. Grenander and G. Szegö, Toeplitz Forms and Their Applications, California Monographs in Mathematical Sciences, University of California Press, Berkeley, 1958.

[33] H. Langer and A. Lasarow, Solution of a multiple Nevanlinna-Pick problem via orthogonal rational functions, Journal of Mathematical Analysis and Applications 293 (2004), no. 2, 605-632.

[34] V. P. Potapov, Linear-fractional transformations of matrices, Studies in the Theory of Operators and Their Applications (Russian), Naukova Dumka, Kiev, 1979, pp. 75-97.

[35] M. Rosenberg, The square-integrability of matrix-valued functions with respect to a non-negative Hermitian measure, Duke Mathematical Journal 31 (1964), no. 2, 291-298.

[36] I. Schur, Über Potenzreihen, die im Innern des Einheitskreises beschränkt sind, I, Journal für die reine und angewandte Mathematik 147 (1917), 205-232.

[37] _ Über Potenzreihen, die im Innern des Einheitskreises beschränkt sind, II, Journal für die reine und angewandte Mathematik 148 (1918), 122-145.

[38] A. Sinap and W. Van Assche, Orthogonal matrix polynomials and applications, Journal of Computational and Applied Mathematics 66 (1996), no. 1-2, 27-52.

[39] G. Szegö, Orthogonal Polynomials, American Mathematical Society Colloquium Publications, vol. 23, American Mathematical Society, New York, 1939.

[40] S. Takenaka, On the orthogonal functions and a new formula of interpolation, Japanese Journal of Mathematics 2 (1925), 129-145.

[41] D. C. Youla and N. N. Kazanjian, Bauer-type factorization of positive matrices and the theory of matrix polynomials orthogonal on the unit circle, IEEE Transactions on Circuits and Systems CAS-25 (1978), no. 2, 57-69.

Andreas Lasarow: Department of Computer Science, Katholieke Universiteit Leuven, Celestijnenlaan 200A, 3001 Heverlee (Leuven), Belgium

E-mail address: andreas.lasarow@cs.kuleuven.be 


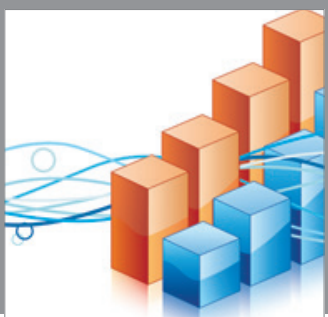

Advances in

Operations Research

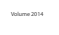

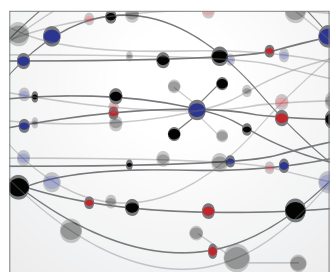

\section{The Scientific} World Journal
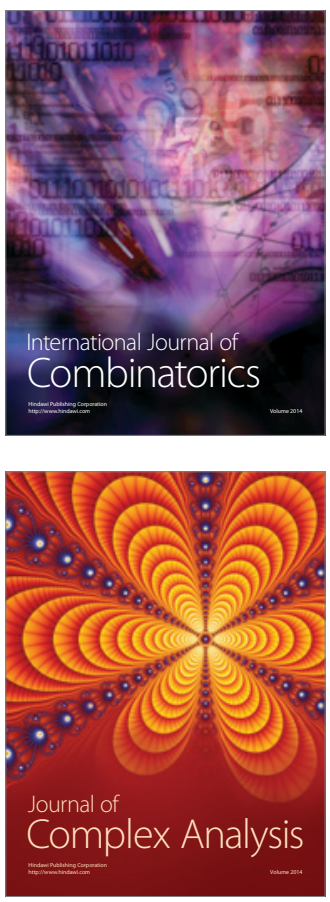

International Journal of

Mathematics and

Mathematical

Sciences
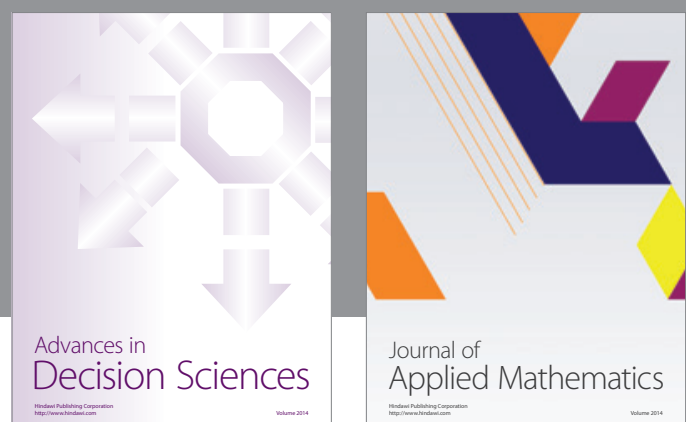

Journal of

Applied Mathematics
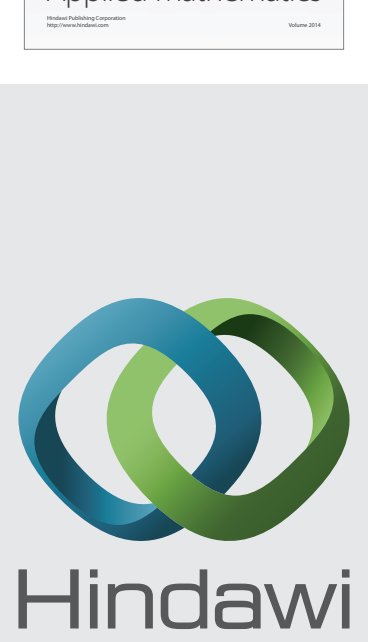

Submit your manuscripts at http://www.hindawi.com
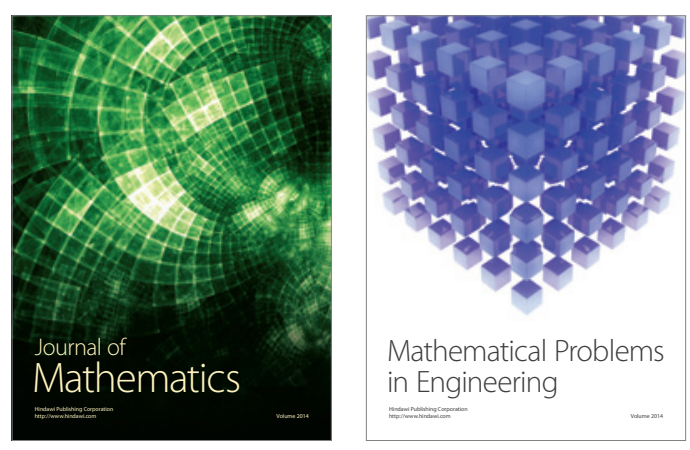

Mathematical Problems in Engineering
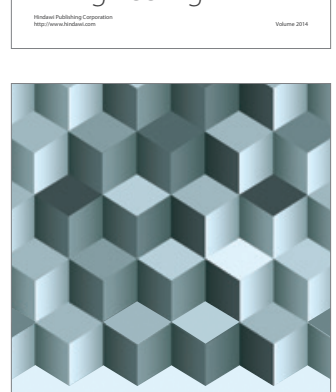

Journal of

Function Spaces
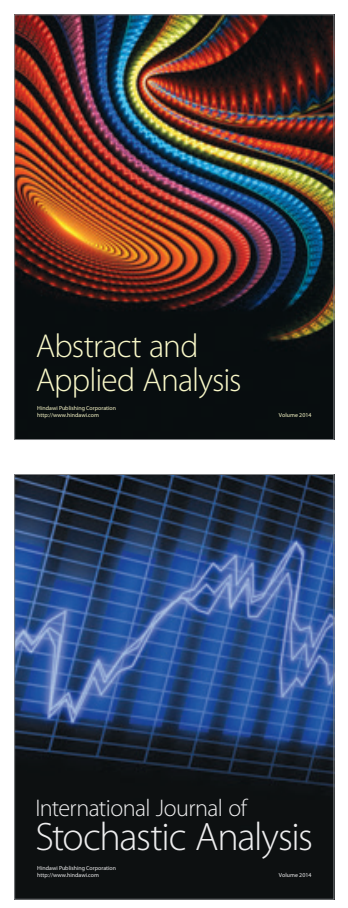

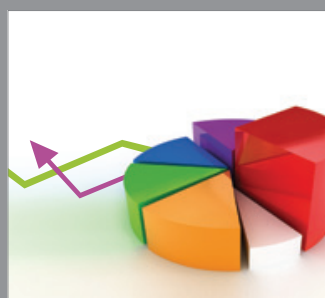

ournal of

Probability and Statistics

Promensencen
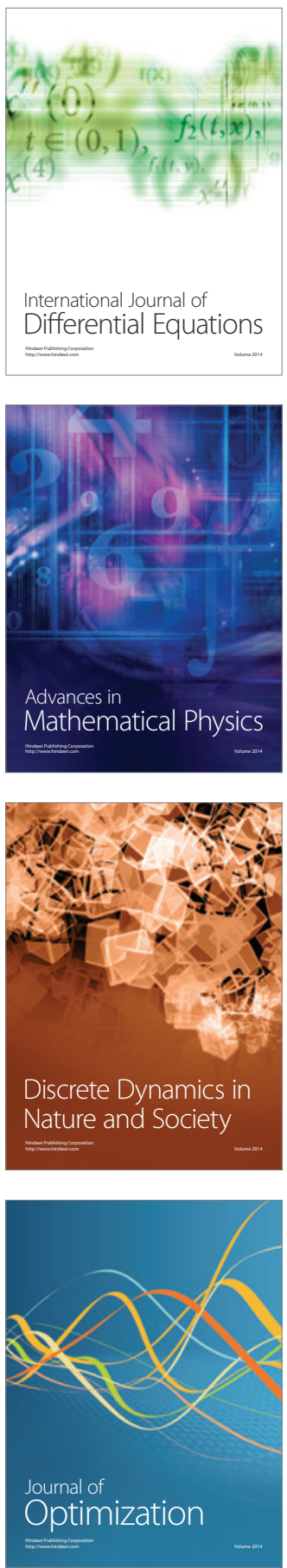